\title{
Micro- and Optoelectronics Fy 2004 Programs and Accomplishments
}
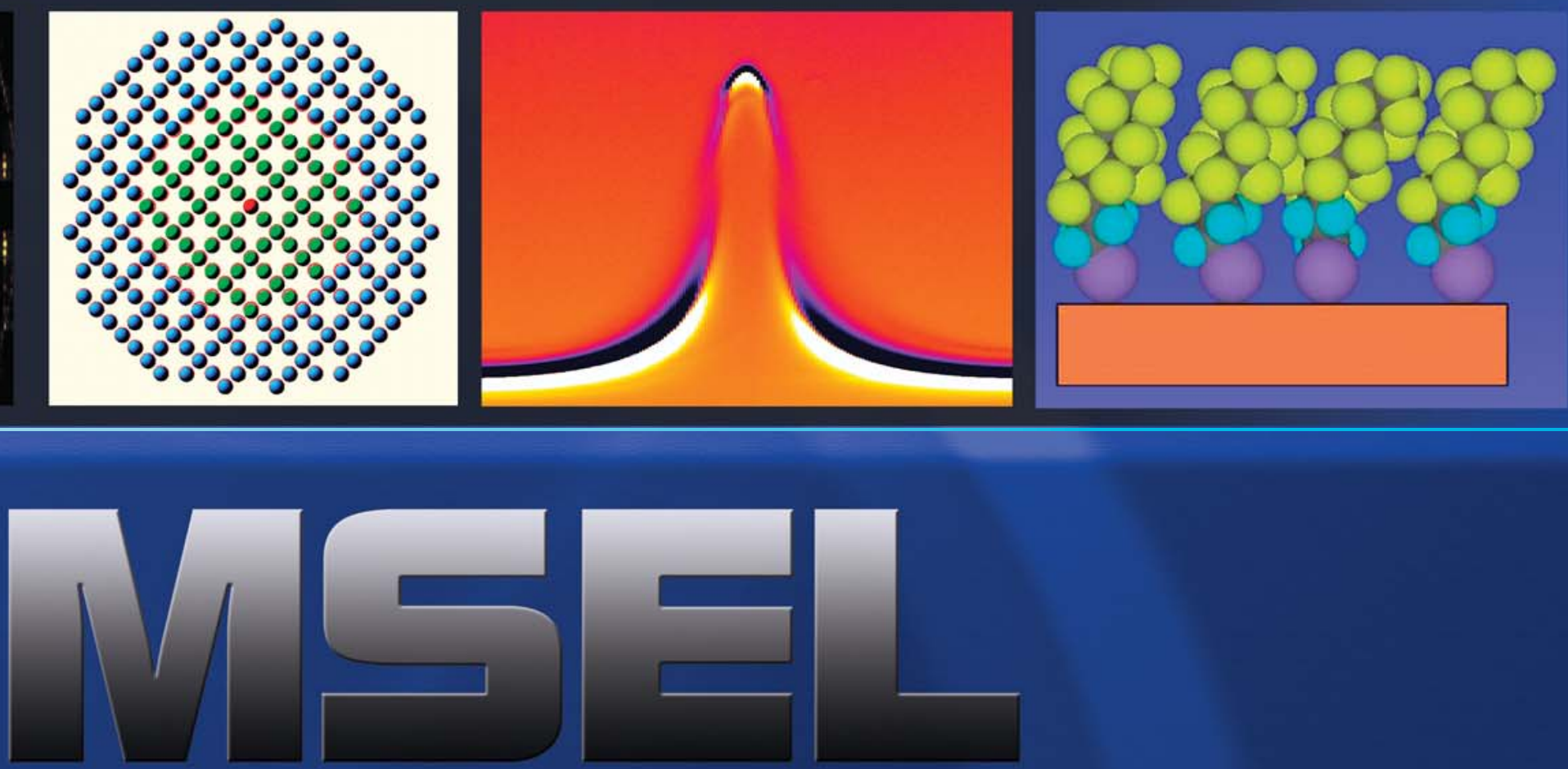

Materials Science and Engineering Laboratory

\section{NGT}

National Institute of

Standards and Technology

Technology Administration

U.S. Department of Commerce

NISTIR 7129

January 2005 


\section{On the Cover:}

On the front cover, from right to left, and continuing onto the back cover, the images shown are, respectively:

Fig. 1 - Schematic illustration of the formation of molecular gradients on elastomeric substrates via self-assembly. (See also the highlight, "Mapping Surface Chemistry and Molecular Orientation with Combinatorial Near Edge X-ray Absorption Fine Structure.”)

Fig. 2 - Ferromagnetic resonance measurements of a Permalloy film on a specially roughened copper substrate as a function of applied field direction. These measurements are part of a study to determine the effects of roughness on magenetization dynamics.

Fig. 3 - Modeling of structure and properties from atomistic to continuum length scales (an atomistic model of a germanium “quantum dot” in silicon).

Fig. 4 - Image displaying the Fourier transform of the average line cross section in a photoresist after etching as measured by critical dimension small angle $\mathrm{x}$-ray scattering (CD-SAXS). 
National Institute of

Standards and Technology

Arden L. Bement, Jr.

Director

Technology

Administration

Phillip J. Bond

Undersecretary of

Commerce for Technology

U.S. Department

of Commerce

Donald L. Evans

Secretary

\section{Materials Science and Engineering Laboratory}

\section{FY 2004 Programs and Accomplishments}

\section{MSEL Materials for Micro- and Optoelectronics}

Eric K. Lin

Leader, Electronics Materials Group

Polymers Division 
Certain commercial entities, equipment, or materials may be identified in this document in order to describe an experimental procedure or concept adequately. Such identification is not intended to imply recommendation or endorsement by the National Institute of Standards and Technology, nor is it intended to imply that the entities, materials, or equipment are necessarily the best available for the purpose. 


\section{Table of Contents}

Executive Summary ............................................................................................................. 1

Technical Highlights ......................................................................................................... 3

First-Principles Studies of Technologically Important Dielectric Materials ..................... 4

Anomalous Elastic Behavior for $\mathrm{SrTiO}_{3}$ Thin Films

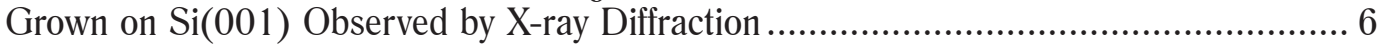

Profiling the Photoresist-Liquid Interface: Fundamentals

for Immersion Lithography and Polymer Dissolution ................................................... 8

On-Chip Interconnects: Extending Performance of Sub-100 nm Lines ...................... 10

Dielectric Metrology and Test Methods Supporting

Embedded Passive Device Technology ................................................................ 12

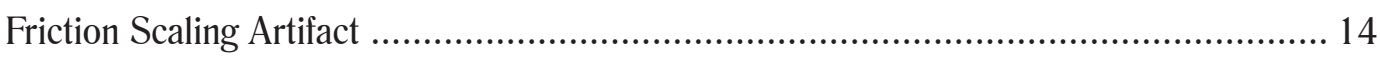

Grand Challenges in Nanomagnetics: High Coercivity FePt

Alloys for Future Perpendicular Magnetic Data Storage .......................................... 16

Breakthroughs in Magnetic Refrigeration ......................................................... 18

Quantitative Nanomechanical Properties ........................................................... 20

Data and Standards Resources ..................................................................... 22

Projects in Materials for Micro- and Optoelectronics ...........................................25

\section{Microelectronics}

Combinatorial Tools for Materials Science …........................................................ 26

Spectroscopy, Diffraction, and Imaging of Electronic Materials ............................... 27

Phase Equilibria and Properties of Dielectric Ceramics ........................................... 28

Theory and Modeling of Dielectric Materials ......................................................... 29

Polymer Photoresists for Next-Generation Nanolithography ..................................... 30

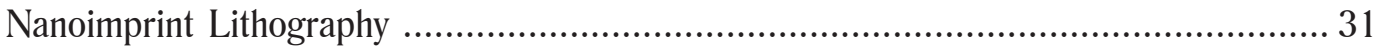

Characterization of Porous Low-k Dielectric Constant Thin Films ............................. 32

Physical Properties of Thin Films and Nanostructures:

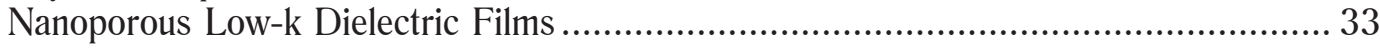

Micrometer-Scale Reliability: Chip-Level Interconnects .......................................... 34

Electrical Properties of On-Chip Interconnections .................................................. 35

Electronic Packaging and Components: Packaging Reliability .................................. 36

Electronic Packaging and Components: Acoustic Characterization ............................ 37

Lead-Free Surface Finishes: Sn Whisker Growth .................................................. 38

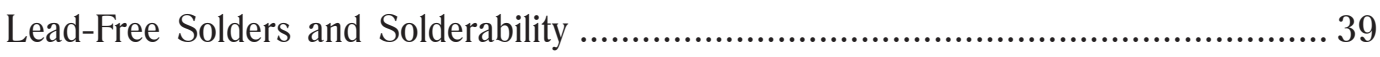

Micrometer-Scale Reliability: Solder Reliability …................................................ 40

Micrometer-Scale Reliability: Mechanical Behavior of Thin Films ............................ 41

Physical Properties of Thin Films and Nanostructures:

Grain Size Effects on Actuator Fatigue 
Experimental and Theoretical Influence of Thin Film Texture

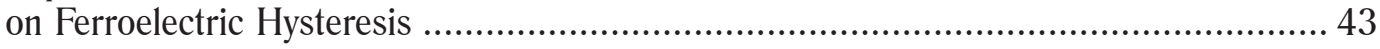

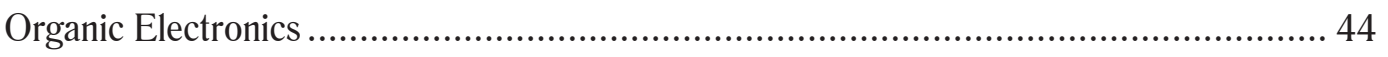

\section{Optoelectronics}

Combinatorial/Phase Diagram Approach for

Metallization to Wide-Band-Gap Semiconductors .................................................. 45

Metrology and Standards for Optoelectronic Materials .......................................... 46

\section{Magnetic Materials}

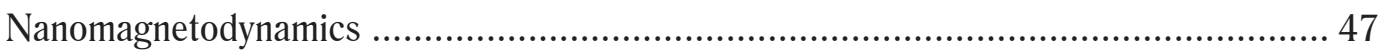

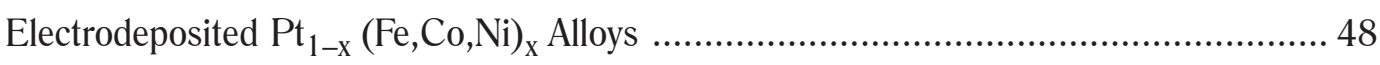

Novel Magnetic Materials for Sensors and Ultra-High Density Data Storage .............. 49

Discovery of Spin Density Waves in a Ferromagnet: Fe-Al ..................................... 50

Micrometer-Scale Reliability: Dynamic Imaging of Magnetic Domain Walls ................ 51

Nanotribology and Surface Properties .................................................................... 52

\section{Related and Cross-Cutting Projects}

Nanoscale Characterization by Electron Microscopy …............................................ 53

Metrology for Nanoscale Properties: X-ray Methods ............................................ 54

Metrology for Nanoscale Properties: Brillouin Light Scattering ................................ 55

Nanostructure Fabrication Processes: Thin Film Stress Measurements ..................... 56

Nanomechanics: Coupling Modeling with Experiments ......................................... 57

Physical Properties of Thin Films and Nanostructures:

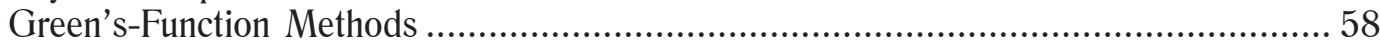

Micrometer-Scale Reliability: Bridging Length Scales ......................................... 59

Micrometer-Scale Reliability: Molecular Dynamics .................................................. 60

Mechanical Metrology for Small-Scale Structures ................................................ 61

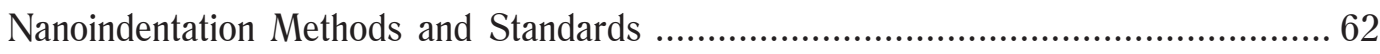

Nanostructure Fabrication Processes:

Patterned Electrodeposition by Surfactant-Mediated Growth ................................. 63

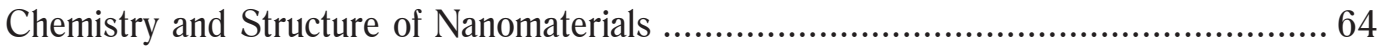

Thermochemistry and Metrology of Interfacial Interactions ......................................6 65

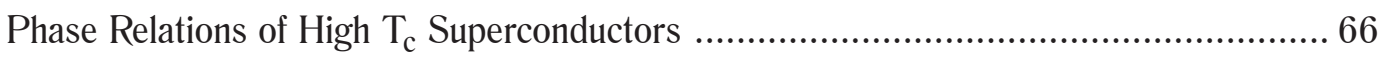

NIST Combinatorial Methods Center (NCMC)

Pioneer and Partner in Accelerated Materials Research ........................................ 67

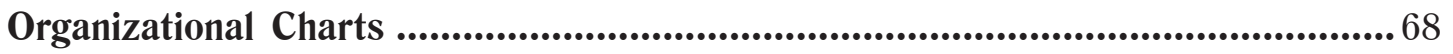




\section{Executive Summary}

\section{Introduction}

The U.S. electronics industry faces strong international competition in the manufacture of smaller, faster, more functional, and more reliable products. Many critical challenges facing the industry require the continual development of advanced materials and processes. The NIST Materials Science and Engineering Laboratory (MSEL) works closely with U.S. industry covering a broad spectrum of sectors including semiconductor manufacturing, device components, packaging, data storage, and assembly, as well as complementary and emerging areas such as optoelectronics and organic electronics.

MSEL has a multidivisional approach, committed to addressing the most critical materials measurement and standards issues for electronic materials. Our vision is to be the key resource within the Federal Government for materials metrology development for commercial microelectronics manufacturing and will be realized through the following objectives:

- Develop and deliver standard measurements and data;

- Develop advanced measurement methods needed by industry to address new problems that arise with the development of new materials;

- Develop and apply in situ as well as real-time, factory floor measurements, for materials and devices having micrometer- to nanometer-scale dimensions;

- Develop combinatorial material methodologies for the rapid optimization of industrially important electronic materials;

- Quantify and provide the fundamental understanding of the divergence of thin film and nanoscale material properties from their bulk values;

- Provide the fundamental understanding of materials needed for future nanoelectronic devices, including first principles modeling of such materials.

The NIST/MSEL program consists of projects led by the Metallurgy, Polymers, Materials Reliability, and Ceramics Divisions. These projects are conducted in collaboration with partners from industrial consortia (e.g., International SEMATECH), individual companies, academia, and other government agencies. The program is strongly coupled with other microelectronics programs within the government such as the National Semiconductor Metrology Program (NSMP). Materials metrology needs are also identified through the International Technology Roadmap for Semiconductors (ITRS), the IPC Lead-free Solder
Roadmap, the National Electronics Manufacturing Initiative (NEMI) Roadmap, the Opto-electronics Industry Development Association (OIDA) Roadmap, IPC (the International Packaging Consortium), and the National [Magnetic Data] Storage Industry Consortium (NSIC) Roadmap.

The present report describes the major technical activities and accomplishments in the area of Materials for Micro- and Optoelectronics within MSEL in FY2004 (October 2003 through September 2004). In this report, we have tried to provide insight into how MSEL research programs meet the needs of our customers, how MSEL capabilities are being used to solve problems important to the national economy and the materials metrology infrastructure, and how MSEL interacts with its customers to establish new priorities and programs. Feedback and suggestions on how we can better serve the needs of our customers and encourage increasing collaboration to this end are welcomed.

\section{Mission}

Our mission is to provide critical leadership in the development of measurement methods and standards, as well as fundamental understanding of materials behavior needed by the U.S. microelectronics and optoelectronics industries to remain competitive in the changing global marketplace. As an essential part of this mission, we are responsible not only for developing new measurement methods with broad applicability across micro- and optoelectronics classes and industries, but also for working with individual industry groups to develop and integrate measurements, standards, software tools, and evaluated data for specific, technologically important applications.

\section{Establishing Priorities}

In the development of this technical program, a wide range of research opportunities is first examined. Choices for the research portfolio are based on several criteria: the match to the NIST mission, the magnitude and immediacy of industrial need, the degree to which NIST's contribution is critical for success, the anticipated impact relative to the investment, ability to respond in a timely fashion with high-quality output, and the opportunity to advance mission-oriented science. This requires that MSEL establish its research priorities through extensive consultation and collaboration with customers in U.S. industry and with counterparts in the international metrology community using a variety of methods 
including workshops, technical meetings, standards committee participation, and individual consultation with our customers. Materials metrology needs are also identified through industry groups and roadmaps, including the International Technology Roadmap for Semiconductors (ITRS), the IPC Lead-free Solder Roadmap, the National Electronics Manufacturing Initiative (NEMI) Roadmap, the Optoelectronics Industry Development Association (OIDA) roadmaps, and the Information Storage Industry Consortium (INSIC).

This program deals almost exclusively with rapidly evolving technologies, where advances in measurement science are needed to understand the limitations on system behavior. MSEL focuses its efforts to address these needs in order to have the best chance to have an impact on the course of technology.

\section{Research Portfolio}

Although there is increasing integration within various branches of microelectronics and optoelectronics, the field can be considered to consist of three main areas. The first, microelectronics, includes needs ranging from integrated circuit fabrication to component packaging to final assembly. MSEL programs address materials metrology needs in each of these areas including, for example, polymer photoresists for next-generation lithography and electrodeposition of interconnects; electrical, mechanical, and physical property measurement of dielectrics (interlevel, packaging, and wireless applications); and packaging and assembly processes (lead-free solders, solder interconnect design, and thermal stress analysis). New areas this year include advanced gate dielectric materials (pp. 4-7, p. 26), nanoimprint lithography (p. 31) methods to quantify nanomechanical properties (pp. 20-21), and organic electronics (p. 44).

The second major area is optoelectronics, which includes work that often crosses over into electronic and wireless applications. Projects currently address residual stress measurement in optoelectronic films, performance of III-V optical materials, and wide bandgap semiconductors. In particular, the wide bandgap program has grown as a collaboration with the NIST Electronic and Electrical Engineering Laboratory (EEEL). Building on the existing projects on metal interconnects for GaN (Metallurgy Division) and on interface and bulk defects in GaN (Ceramics
Division), the EEEL/MSEL program is developing a comprehensive suite of measurement methods, including an integrated, phase-diagram combinatorial approach for characterizing and optimizing interface and bulk defects limiting the application of $\mathrm{GaN}$ and related materials.

A third, complementary area is magnetic data storage, where the market potential is vast and growing, and the technical challenges extreme. INSIC plans to demonstrate a recording density of 1 terabit per square inch - 40 times today's level - by 2006 . To reach these goals, new materials are needed that have smaller grain structures, can be produced as thin films, and can be deposited uniformly and economically. New lubricants are needed to prevent wear as spacing between the disk and head becomes smaller than the mean free path of air molecules. Some measurements require calibration of magnetometers using certified magnetic standards in several different shapes and magnetic strengths, and with a wide range in magnetic character. These standards are now being produced under this program. MSEL is working with the magnetic recording industry to develop measurement tools, modeling software, and standards to help achieve these goals. Staff expertise at MSEL spans all fields relevant to magnetic data storage, including materials science, electrical engineering, physics, mathematics and modeling, manufacturing engineering, chemistry, metrology, and computer science, with the Materials Science and Engineering Laboratory, the Electronics and Electrical Engineering Laboratory, the Physics Laboratory, the Information Technology Laboratory, and the Manufacturing Engineering Laboratory working as partners in this effort.

\section{Project Output}

An integral part of our research programs is the dissemination of our technical accomplishments. Project output exists in a variety of forms, ranging from a fundamental understanding of micro- and optoelectronics behavior to new measurement techniques required for anticipated developments in materials use or design. These outputs are conveyed through the scientific literature and oral presentations, workshops, standard reference materials, standard test method development, evaluated data and online databases, software tools, and sensors for online process control. 


\section{Technical Highlights}

The following Technical Highlights section includes expanded descriptions of research projects that have broad applicability and impact. These projects generally continue for several years. The results are the product of the efforts of several individuals. The Technical Highlights include:

- First-Principles Studies of Technologically Important Dielectric Materials

- Anomalous Elastic Behavior for $\mathrm{SrTiO}_{3}$ Thin Films Grown on $\mathrm{Si}(001)$ Observed by X-ray Diffraction

- Profiling the Photoresist-Liquid Interface: Fundamentals for Immersion Lithography and Polymer Dissolution

- On-Chip Interconnects: Extending Performance of Sub-100 nm Lines

- Dielectric Metrology and Test Methods Supporting Embedded Passive Device Technology

- Friction Scaling Artifact

- Grand Challenges in Nanomagnetics: High Coercivity FePt Alloys for Future Perpendicular Magnetic

Data Storage

- Breakthroughs in Magnetic Refrigeration

- Quantitative Nanomechanical Properties

- Data and Standards Resources 


\section{First-Principles Studies of Technologically Important Dielectric Materials}

\begin{abstract}
Dielectric materials are needed for diverse applications ranging from gate dielectrics to microwave resonator materials to transducer and actuator materials. The specific materials requirements depend on the application. First-principles theoretical studies on dielectric materials are revealing the roles of chemical order and internal electric fields in controlling the properties of these materials.
\end{abstract}

\section{Eric Cockayne and Benjamin P. Burton}

Csic urrent semiconductor technology uses amorphous $\mathrm{SiO}_{2}$ as a gate dielectric material. As size has decreased, however, the leakage current of $\mathrm{SiO}_{2}$ has become a limiting factor. Finding an alternative gate dielectric material is a "grand challenge" in the industry. Alternative materials must have higher dielectric constants than $\mathrm{SiO}_{2}$ to yield equivalent performance with reduced leakage current. Hf-containing oxides are a promising set of materials.

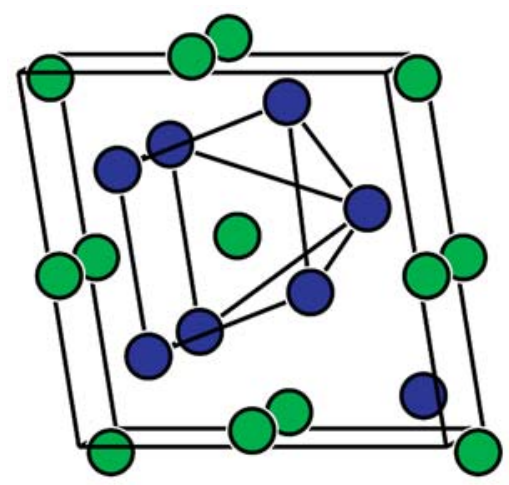

Figure 1: $\mathrm{HfO}_{2}$ structure ( $\mathrm{Hf}$ green, $\mathrm{O}$ blue).

Dielectric constants are related to the effective charges of ions and to phonon-frequencies. First-principles (FP) calculations show that the effective charge of $\mathrm{Hf}$ in $\mathrm{HfO}_{2}$ (Figure 1) is higher than the effective charge of Si in $\mathrm{SiO}_{2}$, providing an explanation why Hf-containing oxides should have, in general, higher dielectric constants than silicates. By comparing the effective charge of $\mathrm{Hf}$ in the experimental structure with that in hypothetical structures, it was found that the effective charge of Hf depends on its environment. Because amorphous gate dielectrics are desirable, knowledge of how crystal environments dictate effective ion-charges provides a basis for designing gate dielectric materials.

Microwave resonator and filter materials typically require a moderate dielectric constant, low dielectric loss,

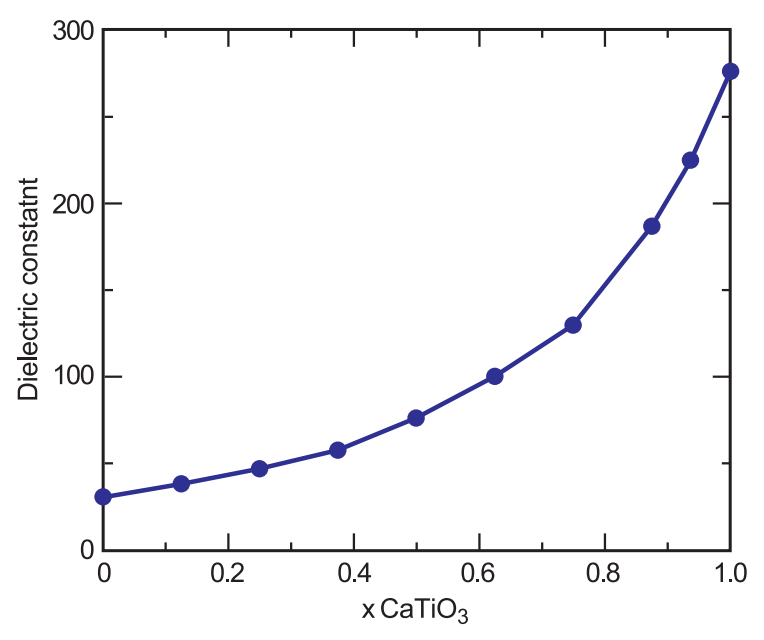

Figure 2: First-principles-derived model for the dielectric constant of $\left(\mathrm{CaAl}_{1 / 2} \mathrm{Nb}_{1 / 2} \mathrm{O}_{3}\right)_{1-x}-\left(\mathrm{CaTiO}_{3}\right)_{x}$ shows nonlinear composition dependence, in agreement with experiment.

and temperature stability. In general, temperature stability is achieved by forming solid solutions between end member compounds with opposite signs of temperature coefficient, for example, between $\mathrm{CaAl}_{1 / 2} \mathrm{Nb}_{1 / 2} \mathrm{O}_{3}(\mathrm{CAN})$ and $\mathrm{CaTiO}_{3}(\mathrm{CT})$. We have used the FP-based model to calculate the dielectric constant of arbitrary solid solutions of $-\mathrm{CaAl}_{1 / 2} \mathrm{Nb}_{1 / 2} \mathrm{O}_{3}-\mathrm{CaTiO}_{3}$. The model is based on cluster expansions of the interatomic forces and ionic charges, which control the dielectric properties.

The model reproduces the experimentally measured composition dependence of the dielectric constant of CT-CAN very well (Figure 2).

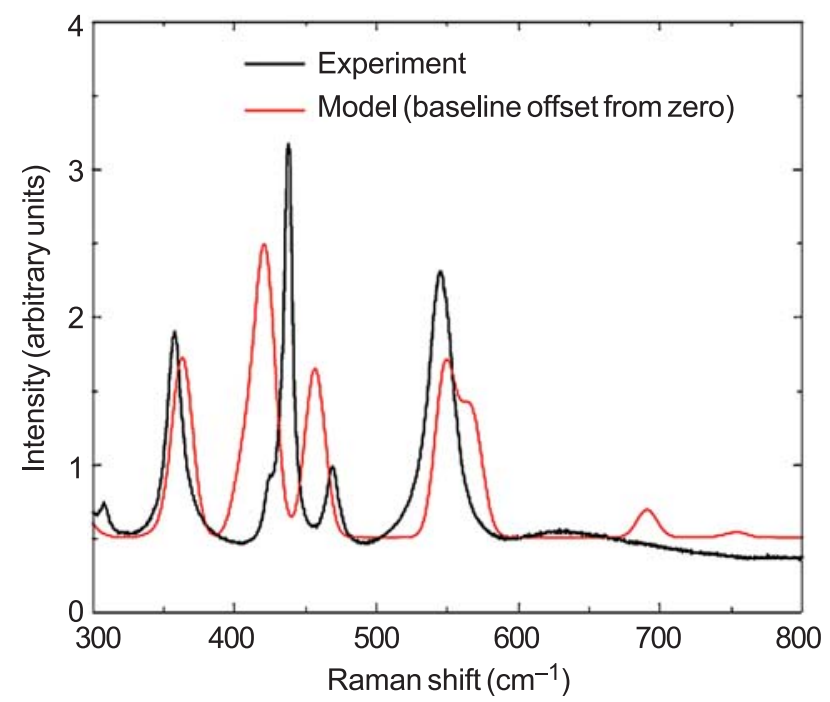

Figure 3: Combined first-principles/empirical model for the Raman spectrum of $\mathrm{CaZrO}_{3}$ shows semiquantitative agreement with experiment. 
Dielectric properties, and, therefore, the Raman spectra, of solid solutions depend on chemical ordering of ions as well as composition. To understand the connection between local structure, composition, dielectric properties, and spectroscopy, we are working on computing Raman spectra directly from FP. The idea is to create a spectroscopic/computational tool for measuring short- and long-range chemical order via Raman spectroscopy. Experiments have shown that Raman spectra are sensitive to short- and long-range order, but little is known quantitatively about the relationship between Raman spectra and chemical order. We have created a model, based on FP results, for the Raman spectra of solids, and are applying it to study the CAN-CT and $\mathrm{CaZrO}_{3}-\mathrm{CaTiO}_{3}(\mathrm{CZ}-\mathrm{CT})$ solid solution systems. Figure 3 shows that the Raman spectrum of $\mathrm{CZ}$ is reproduced reasonably well with only a few parameters. Both long-range electrostatics and short-range covalent bonding contribute to the Raman spectra, but not equally to all peaks. This provides a natural explanation for the experimental observation that different Raman peaks can have different sensitivities to the amount of long-range order. Evidence is also found that small $\mathrm{Ti}$ or $\mathrm{Zr}$ concentrations create local vibrations that produce significant, but broad, peaks in the Raman spectrum.

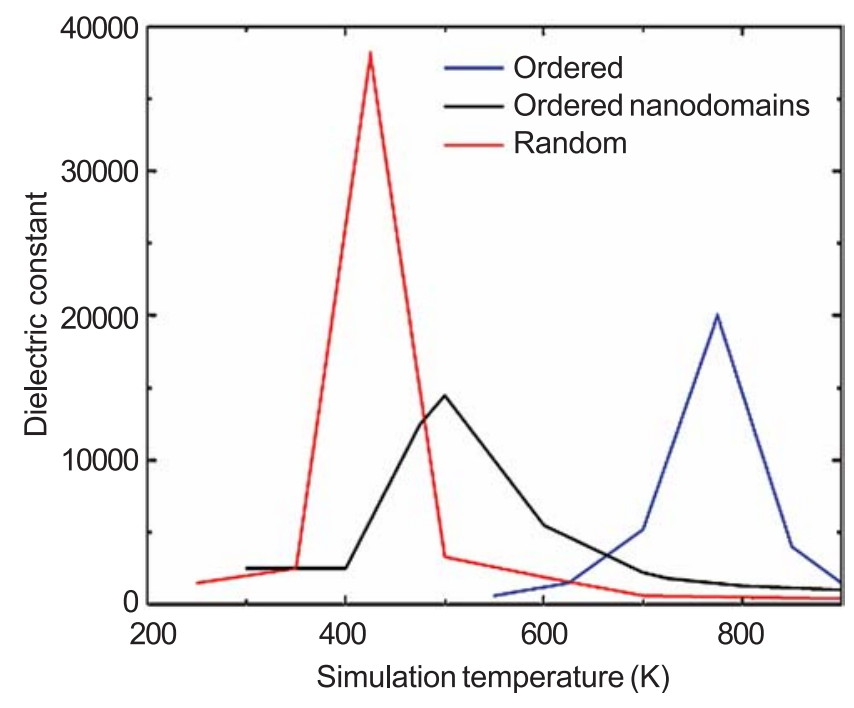

Figure 4: Simulated dielectric constant of $\mathrm{PbSc}_{1 / 2} \mathrm{Nb}_{1 / 2} \mathrm{O}_{3}$ as a function of temperature and type of $\mathrm{Sc}-\mathrm{Nb}$ ordering. The broadening of the dielectric peak that occurs in the case of ordered nanodomains is one characteristic of relaxor behavior.

Transducers used for sonar and medical imaging applications typically require extremely high dielectric and piezoelectric coefficients. Very large piezoelectric coefficients are observed in $\mathrm{Pb}[\mathrm{Mg} ; \mathrm{Zn}]_{1 / 3} \mathrm{Nb}_{2 / 3} \mathrm{O}_{3}-$ $\mathrm{PbTiO}_{3}$ solid solutions, based on the relaxor ferroelectrics $\mathrm{PbMg}_{1 / 3} \mathrm{Nb}_{2 / 3} \mathrm{O}_{3}$ and $\mathrm{PbZn}_{1 / 3} \mathrm{Nb}_{2 / 3} \mathrm{O}_{3}$.
Relaxor behavior is desirable, because it implies high-dielectric constants and piezoelectric coefficients over a broad temperature range, but it poses a theoretical challenge because these properties are sensitive to structural/chemical heterogeneities at many length-scales. For example, $\mathrm{PbSc}_{1 / 2} \mathrm{Nb}_{1 / 2} \mathrm{O}_{3}$ (PSN) exhibits normal ferroelectric behavior as a function of temperature (e.g., a sharp dielectric peak) when $\mathrm{Sc}^{3+}$ and $\mathrm{Nb}^{5+}$ are ordered. However, when there is only short-range order, such as nanoscale chemically ordered domains in a disordered matrix, PSN exhibits relaxor ferroelectric behavior (e.g., a broadened, frequency-dependent dielectric peak). Similar changes in dielectric properties are caused by the introduction of $\mathrm{Pb}$ - and $\mathrm{O}$-vacancies. We have constructed a computational model for PSN, which allows these structure-property issues to be explored in detail. FP calculations show that $\mathrm{Pb}$ atoms move off their ideal crystallographic positions, and that the dielectric and piezoelectric response in PSN is dominated by the dynamics of $\mathrm{Pb}$-displacements. At low temperature, $\mathrm{Pb}-\mathrm{Pb}$ interactions favor coherent alignments of $\mathrm{Pb}$-displacements, but local electric fields, caused by the charge difference between $\mathrm{Sc}^{3+}$ and $\mathrm{Nb}^{5+}$, randomly bias individual $\mathrm{Pb}$-displacements. Electric fields generated by $\mathrm{Pb}$ - and $\mathrm{O}$-vacancies are also included in this model. Molecular dynamics simulations on a 320,000 atom supercell yield a sharp dielectric peak for ordered PSN, and successive broadening as chemically ordered nanodomains and/or $\mathrm{Pb}$ vacancies are added, in agreement with experiment.

The wide band-gap semiconductor system AlN-GaN is used to make light emitting diodes, and the wavelength of emitted light is adjusted by alloying. First-principles phase diagram calculations for this system predict the expected miscibility gap when the excess vibrational entropy is excluded; but when it is included, an ordered phase based on $\mathrm{AlGaN}_{2}$ stoichiometry is predicted. To our knowledge, this is the first prediction of vibrational-entropy induced atomic ordering, and it suggests a novel way to affect band gaps: via ordering and alloying rather than alloying alone.

\section{For More Information on this Topic}

E. Cockayne, B.P. Burton (Ceramics Division, NIST)

E. Cockayne, "First-principles Calculations of the Dielectric Properties of Perovskite-Type Materials," J. Eur. Ceram. Soc. 23, 2375-2379 (2003).

U.V. Waghmare, E. Cockayne, and B.P. Burton, "Ferroelectric Phase Transitions in Nano-scale Chemically Ordered $\mathrm{PbSc}_{0.5} \mathrm{Nb}_{0.5} \mathrm{O}_{3}$ using a First-principles Model Hamiltonian," Ferroelectrics 291, 187-196 (2003). 


\section{Anomalous Elastic Behavior for $\mathrm{SrTiO}_{3}$ Thin Films Grown on Si(001) Observed by X-ray Diffraction}

A major driving force of the semiconductor industry is the integration of transition-metal oxides with Si transistor technology. Much of this effort has focused on the development of $\mathrm{SrTiO}_{3}$ thin-film growth on $\mathrm{Si}(001)$. These films have served successfully as the gate-oxide layer for metal-oxide field-effect transistors and as the buffer layer for III-V on Si semiconductor technology. In addition, ferroelectric thin films show promise for quantum computing applications. However, these perovskite films exhibit unusual features, and it is imperative for future advances that the fundamental aspects of the thin-film epitaxy be understood from both an experimental and a theoretical view. To address this issue, we are studying $\mathrm{SrTiO}_{3}$ as a model material representative of the larger class of candidate oxides with the perovskite structure.

\section{Joseph C. Woicik}

$\mathrm{W}$ e have performed high resolution $\mathrm{x}$-ray diffraction measurements at the UNICAT beamline facility at the Advanced Photon Source on a series of $\mathrm{SrTiO}_{3}$ thin films grown on $\mathrm{Si}(001)$ by Motorola. Figure 1

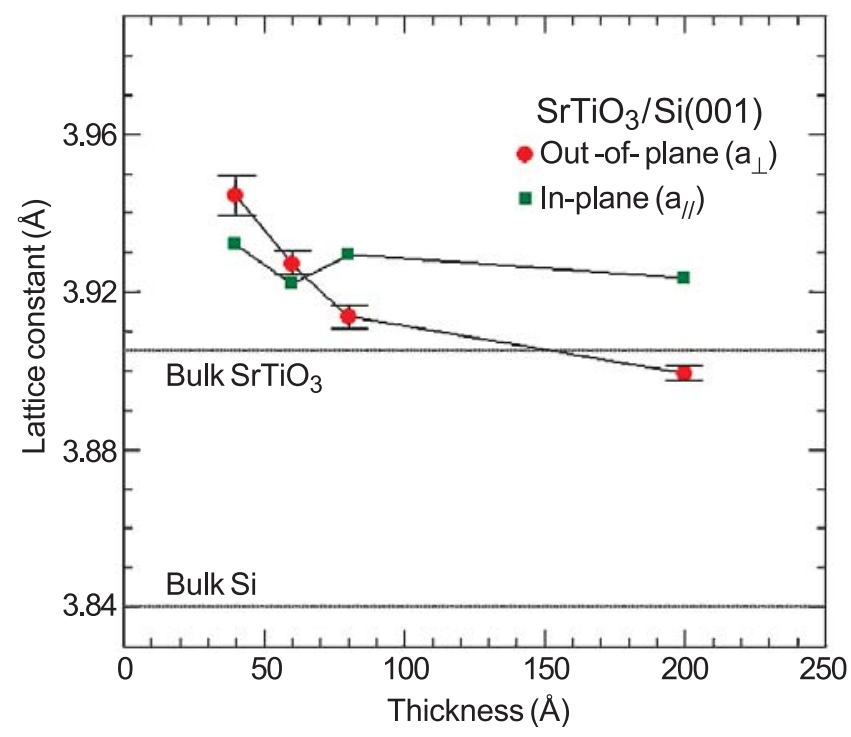

Figure 1: In-plane and out-of-plane $\mathrm{SrTiO}_{3}$ lattice constants for $40 \AA, 60 \AA, 80 \AA$, and $200 \mathrm{~A} \mathrm{SrTiO}_{3}$ thin films grown on $\mathrm{Si}(001)$. The horizontal lines show the bulk cubic lattice constants of $\mathrm{SrTiO}_{3}$ and bulk $\mathrm{Si}$. shows the results of our glancing-incidence x-ray diffraction experiment performed around the $\mathrm{SrTiO}_{3}(220)$ diffraction for the in-plane and the $\mathrm{SrTiO}_{3}(002)$ diffraction for the out-of-plane $\mathrm{SrTiO}_{3}$ lattice parameters.

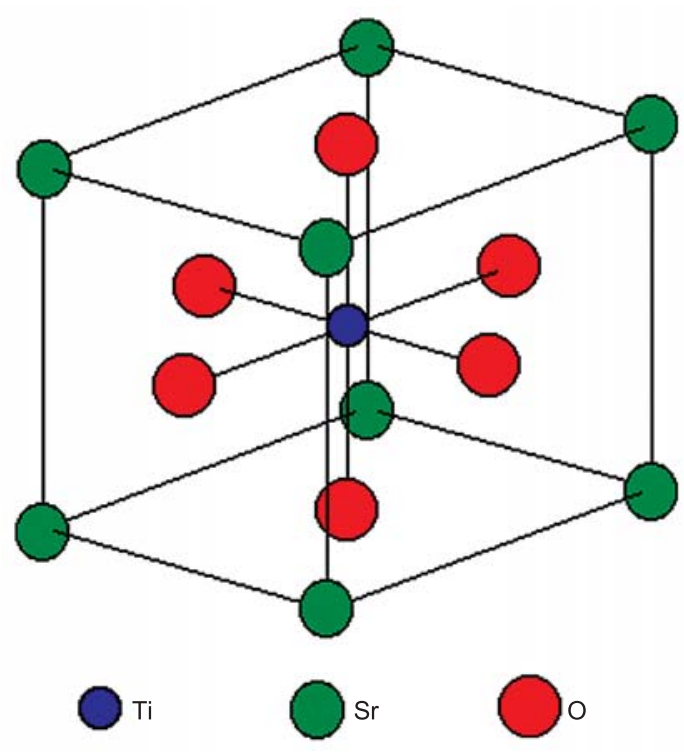

Figure 2: Crystal structure of $\mathrm{SrTiO}_{3}$.

Because the crystal structure of $\mathrm{SrTiO}_{3}$ is simple cubic (Figure 2), whereas the crystal structure of $\mathrm{Si}$ is face-centered cubic, $\mathrm{SrTiO}_{3}$ films grow on the $\mathrm{Si}(001)$ surface with their unit cell rotated $45^{\circ}$ around the $\mathrm{Si}(001)$ surface-normal direction. Consequently, this choice of $\mathrm{SrTiO}_{3}$ reflections determines accurately the $\mathrm{SrTiO}_{3}$ unit cell dimensions relative to the underlying $\mathrm{Si}$ lattice and hence the thin film epitaxy.

Our diffraction measurements find that all of the thin films studied are under in-plane tensile strain. This finding is counter to what is expected from the bulk lattice constants of $\mathrm{SrTiO}_{3}$ and $\mathrm{Si}$ that predict in-plane compressive strain for coherent $\mathrm{SrTiO}_{3}$ thin-film growth on $\mathrm{Si}(001)$. The observed expansion of the in-plane $\mathrm{SrTiO}_{3}$ lattice constant must therefore result from the much larger thermal expansion coefficient of $\mathrm{SrTiO}_{3}$, compared to $\mathrm{Si}$, coupled with incoherent growth at the elevated growth temperature. 
Even more surprising is the result that the $\mathrm{SrTiO}_{3}$ out-of-plane lattice constant increases with decreasing film thickness, indicating a large negative Poisson's ratio for the thinnest films; i.e., the out-of-plane lattice constant of the $\mathrm{SrTiO}_{3}$ is expanded more than its in-plane lattice constant.

To understand both the nature and driving force of this anomaly, we performed polarization dependent $\mathrm{x}$-ray absorption fine-structure (XAFS) measurements on the same samples. Together, XAFS and x-ray diffraction determine uniquely the degree of epitaxy and the local atomic distortions that result.

Our XAFS measurements find significant $\mathrm{p}-\mathrm{d}$ hybridization for the thinner films. This hybridization is allowed only for a non-centrosymmetric distortion of the $\mathrm{SrTiO}_{3}$ unit cell. Polarization-dependent measurements of the Ti-O bond lengths find one Ti-O distance parallel to the $\mathrm{SrTiO}_{3}$ interface and two Ti-O distances perpendicular to it. The Ti-O radial splitting is $\approx 0.22 \AA$. The elastic anomaly observed by diffraction is, therefore, likely driven by the interfacial polarization of the $\mathrm{SrTiO}_{3}$ layers.

To test this hypothesis, first-principles density-functional calculations were performed on periodic $\mathrm{SrTiO}_{3}$ supercells containing $\sqrt{ } 2 \mathrm{x} \sqrt{2} \times \mathrm{N}$ unit cells. The calculations were performed at the Naval Research Laboratory Center for Computational Materials Science. Figure 3 shows the results of the calculations as a function of $\mathrm{N}$, the number of $\mathrm{SrTiO}_{3}$ layers. The in-plane lattice constant of the supercells was constrained to equal the average in-plane lattice constant of the $\mathrm{SrTiO}_{3}$ films as determined by x-ray diffraction. To mimic the polarization of the interface as determined by XAFS, a single Ti-O layer was polarized in the normal direction by constraining its z-coordinates to lie $0.22 \AA$ apart. All other coordinates were fully relaxed.

Remarkably, the out-of-plane lattice constant of the supercells is found to expand in agreement with

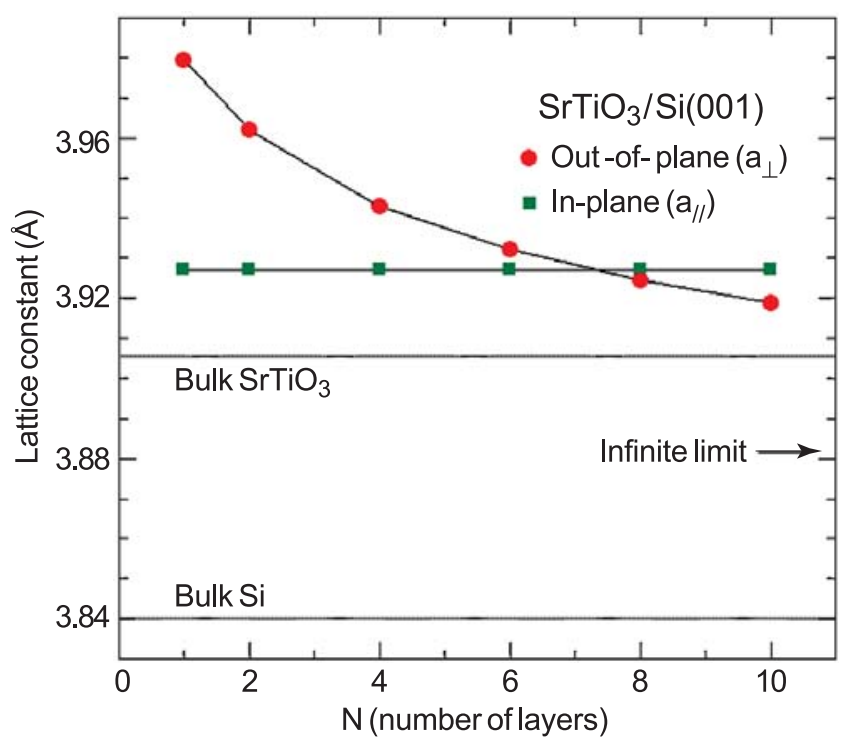

Figure 3: Calculated out-of-plane $\mathrm{SrTiO}_{3}$ lattice constant as a function of the number of $\mathrm{SrTiO}_{3}$ layers.

the measurement of the out-of-plane lattice constant of the films. These results suggest that this behavior may be common for hetero-epitaxial growth of materials that possess an ionic polarizability; it arises simply from the inequivalent perpendicular heights of the anions and cations at the film/substrate interface which drives the polarization of the film.

Additional calculations are being performed to understand the role of $\mathrm{Si}$ diffusion and $\mathrm{O}$ vacancies, and experiments are planned to study both the epitaxy and interfacial structure of films grown under different growth conditions.

\section{For More Information on this Topic}

F.S. Aguirre-Tostado, A. Herrera-Gomez (CINVESTAV); R. Droopad, Z. Yu (Motorola); D.G. Schlom (Pennsylvania State University); P. Zschack, E. Karapetrova (UNICAT); C.S. Hellberg (NRL) 


\section{Profiling the Photoresist-Liquid Interface: Fundamentals for Immersion Lithography and Polymer Dissolution}

Recent advancements in the semiconductor industry have resulted in new problems involving the photoresist-liquid interface. For immersion lithography, the water profile within a resist film impacts pattern quality from changes in photoacid generator diffusion or optical transparency. For the development step, where a latent image is realized into the final structure, an improved understanding of photoresist swelling and dissolution mechanisms is needed to address stringent line-edge roughness requirements. Data from neutron reflectivity measurements provide critical insight needed to understand and optimize next-generation photoresists and process strategies.

\section{Bryan D. Vogt and Vivek M. Prabhu}

\section{Introduction}

$\mathrm{P}$ olymer thin film photoresists comprise the materials foundation for the production of semiconductor devices with nanoscale dimensions. The extension of optical methods has been problematic due to challenges arising from the implementation of shorter exposure wavelengths. The past focus of the semiconductor industry has been the development of sufficiently transparent photoresist materials for future exposure sources. However, future progress requires depth profile information at the photoresist-liquid interface, due to the emergence of immersion lithography and the increased influence of the photoresist development process on lithographic performance.

Using neutron reflectivity (NR), NIST successfully quantified the profile of water and aqueous base counterions in model photoresist films. NR provides structural information regarding the composition profile normal to the thin-film surface with isotopic selectivity between protons and deuterium. Selective deuteration of components in the system allows for the quantification of water or counterion distribution within photoresist films despite the negligible differences in physical density. This data provide critical insight needed to refine models for immersion lithography and polymer dissolution.

\section{Immersion Lithography: Water Profile}

Recently, immersion lithography has emerged as the key strategy to extend existing optical tools. A liquid, such as water, is placed between the lens and photoresist thin film to enhance resolution.
The industry anticipates using immersion lithography for production in 2007 at the $65 \mathrm{~nm}$ node. The role of liquids in contact with photoresist films is important; not only for component leaching and contamination, but also due to the detrimental influence of trace levels of water on the reaction and diffusion of photoacid generators. Additionally, a non-uniform water profile within thin films leads to incorrect assumptions regarding the transmission and reflection at the photoresist-anti-reflective coating interface.

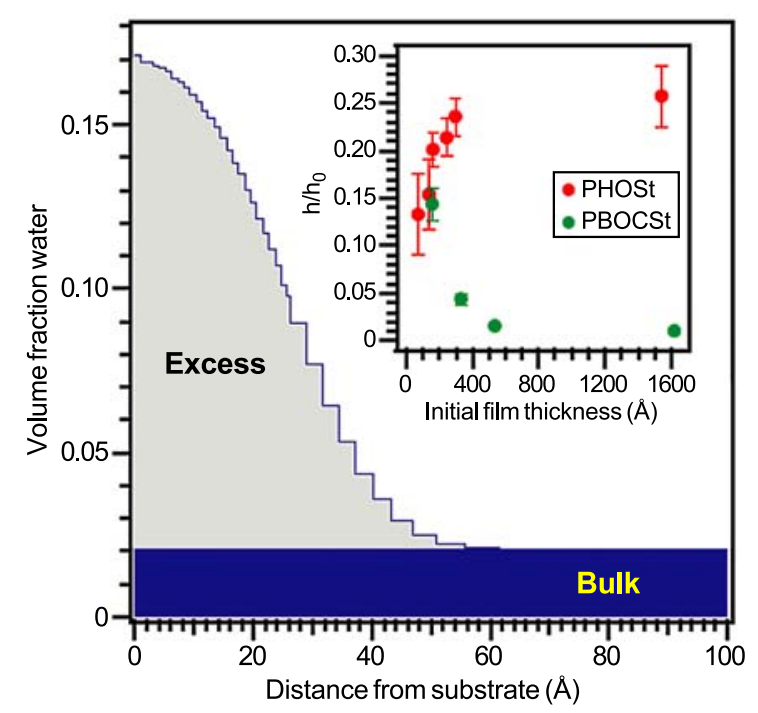

Figure 1: Volume fraction of water distributed near the PBOCSt/HMDS treated substrate during immersion, as determined by neutron reflectivity. The excess of water at the substrate reaches a maximum concentration of $17 \%$ by volume. Inset: Dependence of initial film thickness on the total film swelling for two model photoresists in thin and ultrathin films.

Figure 1 shows the measured volume fraction profile of water versus the distance from a trimethylsilane-primed silicon oxide interface. Far from the substrate, the $248 \mathrm{~nm}$ photoresist, poly(4-tert-butoxycarbonlyoxystyrene) (PBOCSt), shows bulk water absorption near $2.5 \%$. However, significant deviations occur near the interface. An excess of water, up to $17 \%$, extends $40 \AA$ from the substrate. This enriched interfacial water was previously unknown and unaccounted for.

In contrast, a depletion of water was observed for poly(4-hydroxystyrene) (PHOSt), the developer soluble resist, even though the bulk of the film absorbs $25 \%$. This depletion indicates the interface cannot accommodate excess water. It appears that the relative hydrophobicity between polymer and substrate controls the amount of interfacial water. 
The total film swelling is also observed to be a function of the initial thickness and interfacial water. For thinner resist films the interfacial water dominates the swelling as shown in the inset to Figure 1 for PBOCSt and PHOSt. For these different resists, the swelling becomes similar for ultrathin films with thickness less than $200 \AA$. The data are consistent with an interfacial water thickness and content for each film thickness. The interfacial concentration is strongly dependent upon the surface chemistry, but relatively independent of the photoresist material or film thickness.

\section{Dissolution Effects: Aqueous Base Profile}

The development step of a latent image in an aqueous base contributes significantly to undesirable line-edge roughness (LER). There is a strong need for improved dissolution models incorporating photoresist-developer interactions. A key component is the depth profile of the aqueous base counterion through a photoresist film because it controls the dissolution mechanisms that lead to unacceptable LER. We provided the first direct measurement of the aqueous base distribution (tetramethylammonium hydroxide) (TMAH) within the film. These measurements quantify the extent of developer penetration and the influence of ionization on the response of the photoresist to the developer solution.

We used a zero-average contrast (ZAC) experiment where the neutron scattering length density of a thin film of poly(norbornene hexafluoroisopropanol) (PNBHFA), a model $157 \mathrm{~nm}$ photoresist, is matched to the developer solution with a $\mathrm{D}_{2} \mathrm{O} / \mathrm{H}_{2} \mathrm{O}$ mixture. As shown in the schematic of Figure 2, the contrast matched film and solvent (equal color) eliminates neutron contrast at this interface. However, when base $\left(d_{12}-T M A H\right)$ enters the film, the reflectivity contrast is enhanced. These changes allow quantification of both film swelling and the base profile through the film.
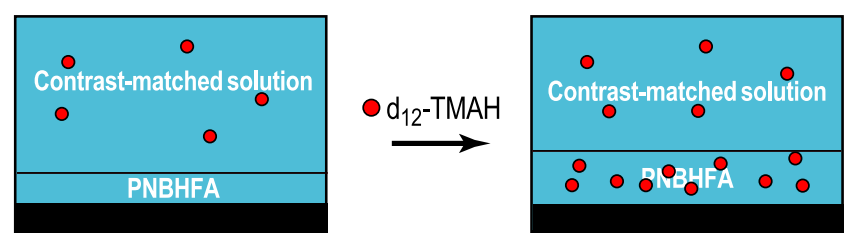

Figure 2: Schematic of the zero-average neutron reflectivity experiment. The contrast between the film and solvent are equal, only the enrichment of deuterated aqueous base within the film provided the reflectivity enhancement.

The volume fraction of $\mathrm{d}_{12}-\mathrm{TMA}^{+}$is plotted versus distance from the substrate in Figure 3 for four different equilibrating concentrations of base. The change in the counterion profile illustrates the advancement of the swollen solid front. A key finding was that the film expansion proceeds via base transport throughout the entire film rather than gradually through the film. In addition, a depletion of base was observed at the substrate. The film-solution interfacial width increases with higher base concentration. These measurements show that ionization-induced swelling occurs at the dissolution front. At even higher base concentrations, the photoresist film dissolves quickly. Understanding the transition from swelling to rapid dissolution with base concentration will provide guidance into developer-induced roughness.

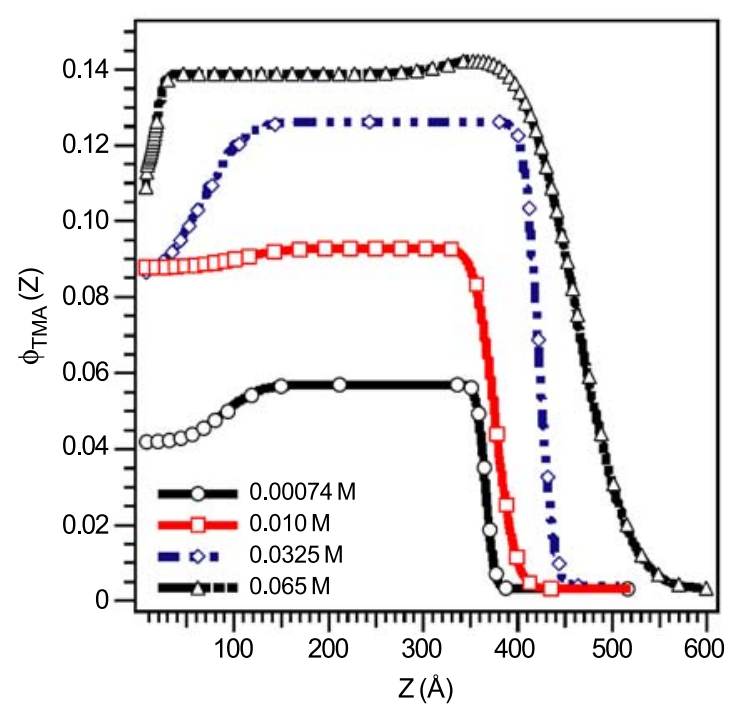

Figure 3: Volume fraction profiles of $d_{12}-\mathrm{TMA}^{+}$within the PNBHFA film. The aqueous base profile illustrates the enhanced swelling and content within the thin film with higher equilibrating base concentration.

These base profilometry experiments were followed by a deswelling step by rinsing with pure water. The rinse kinetically traps base within the film where it could contaminate other processes during device fabrication.

NR measurements provide new information about the liquid-photoresist interface needed to improve current models of dissolution. In future work, we will examine the effects of liquid-photoresist interactions on surface roughness with atomic force microscopy measurements of surface features.

\section{For More Information on this Topic}

C. Soles, M. Wang, C. Wang, R. Jones, W. Wu, E. Lin (Polymers Division, NIST); S. Satija (NIST Center for Neutron Research); D. Goldfarb, M. Angelopoulos (IBM T.J. Watson Research Center); H. Ito (IBM Almaden Research Center)

B.D. Vogt, et al., "Water immersion of model photoresists: Interfacial influences on water concentration and surface morphology," J. Microlithography, Microfabrication, and Microsystems, in press. 


\section{On-Chip Interconnects: Extending Performance of Sub-100 nm Lines}

\begin{abstract}
Conductors in on-chip metallizations are now reaching dimensions so small that defective seed layers are impacting manufacturing yields, and electron scattering on surfaces and grain boundaries is reducing electrical transport in the buried wires. Our goal is to provide tools to overcome these barriers. Recent efforts have quantified sources of the increased resistivity in wires made of silver, the most conductive of all metals, demonstrated seedless processing routes for copper wires, and improved understanding and modeling of the superfill fabrication process.
\end{abstract}

\section{Daniel Josell and Thomas P. Moffat}

$\mathrm{T}_{\mathrm{i}}^{\mathrm{s}}$ he steady reduction of transistor dimensions in integrated circuits has been accompanied by similar size reductions of the on-chip interconnects that carry electrical signals, pushing the industrial technology for copper seed deposition close to its limit for defect-free sidewall coverage. Defects in seed layers, which arise from limitations in existing sputter technology, lead to voiding during electrodeposition of the copper metallization.

Additionally, with the dimensions of the copper wires in these metallizations now approaching the intrinsic mean-free-path length of the conduction electrons, scattering on the wire surfaces has begun to significantly reduce electron transport and, thus, the associated electrical conductance of the wires. With grains in these conductors similarly sized, a reduction is also to be expected from grain boundary scattering. While the penalty for both effects is increased power dissipation and reduced clock speed, the appropriate approach for mitigation requires quantitative determination of the relative sizes of the contributions.
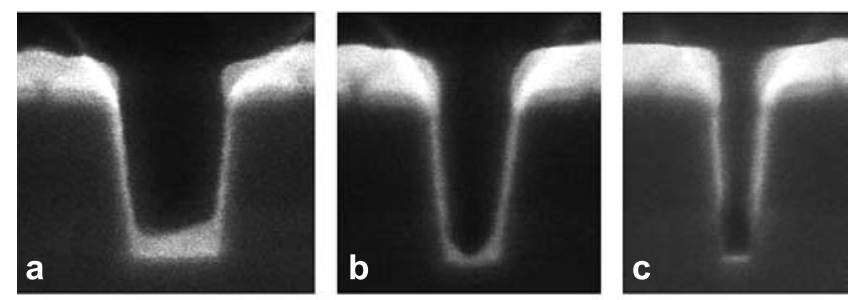

$$
100 \mathrm{~nm}
$$

Figure 1: $\mathrm{Ti} / \mathrm{Pd} / \mathrm{Ag}$ seed layer in sub-100 $\mathrm{nm}$ deep trenches.

\section{Technical Details}

A tri-layer titanium, palladium and silver seed (Figure 1) was shown to yield smooth, conductive surfaces for the electrodeposition of silver wires for the electrical properties study. The poor seed coverage that is visible toward the bottom of the smallest trench (Figure 1c) is a technical challenge noted in the International Technology Roadmap for Semiconductors. Such seed defects motivated the "seedless" ruthenium barrier-based process detailed in last year's report and continued by this year's demonstration of seedless copper superfill in trenches with ruthenium or iridium barriers (Figure 2) deposited by perfectly conformal atomic layer deposition (ALD).

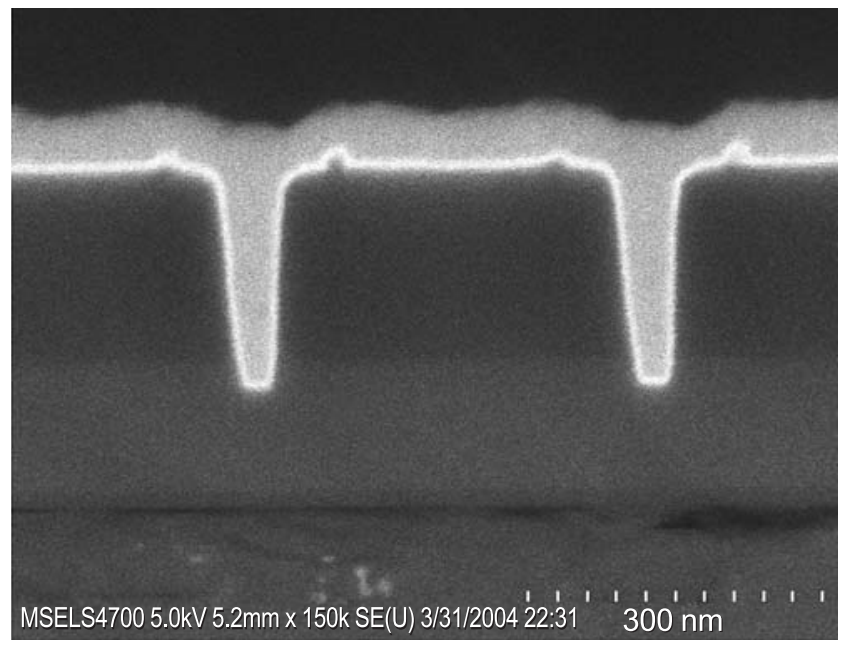

Figure 2: Trenches containing copper that was electrodeposited directly on an ALD iridium barrier (thin bright layer).

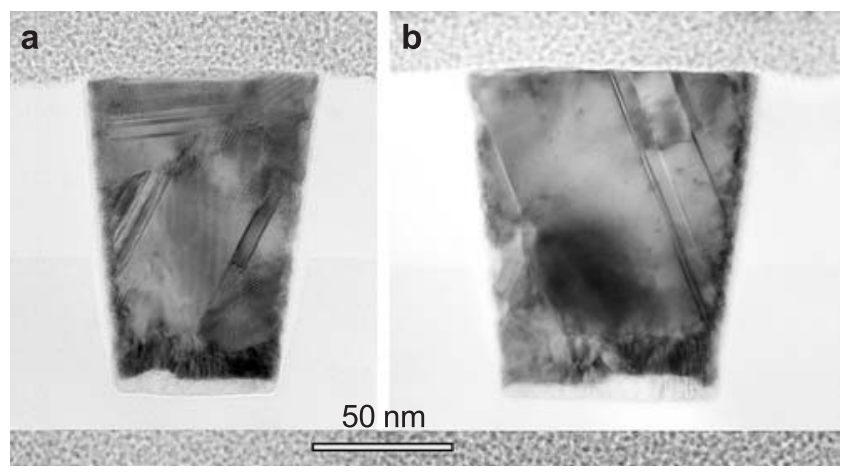

Figure 3: Cross-section views of silver wires fabricated by electrodeposition in the seeded trenches followed by removal of the metal from the field (transmission electron microscope). 
For the electrical studies, silver wires (Figure 3) were fabricated by silver electrodeposition on the tri-layer seeds (Figure 1), using a superfill process developed in the Metallurgy Division, followed by removal of the metal in the field adjacent to the wires through chemicalmechanical planarization and ion polishing.

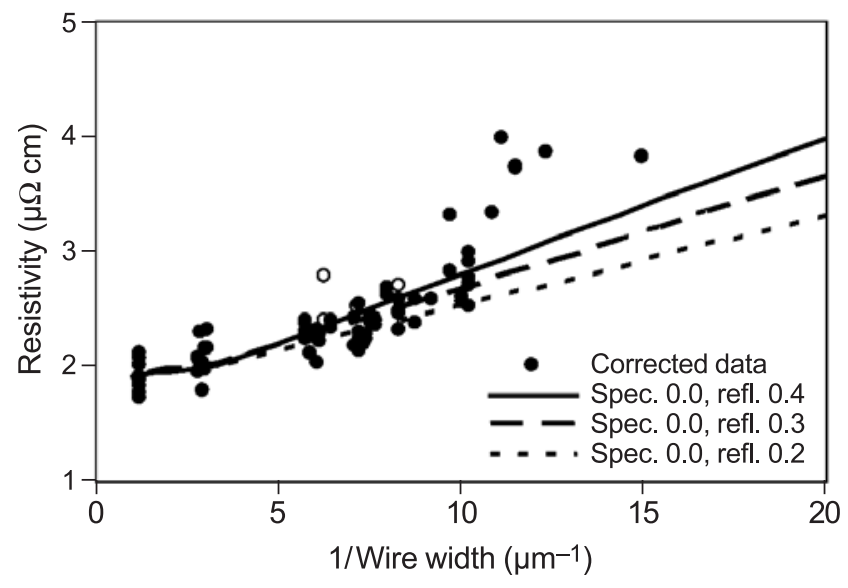

Figure 4: Electrical resistivities of $300 \mathrm{~nm}$ tall silver wires compared to predictions that account for surface scattering with varying amounts of grain boundary scattering. Resistivities for wires less than $100 \mathrm{~nm}$ wide are impacted by defects.

The wires were studied in a standard four-point probe geometry that permitted measurement of wire resistances. Resistivities, obtained from the resistances using measured wire dimensions, increased significantly with decreasing wire width (Figure 4). To assess the origin of the resistivity increase, the Fuchs-Sondheimer analysis for diffuse scattering of electrons on surfaces was extended to permit analysis in the presence of both specular and diffuse scattering. The resulting equations, along with a previously published equation for grain boundary scattering, permitted quantitative evaluation of the experimental data. Significantly, modeling of the data showed that the increase of resistivity with decrease of wire size arises as much from scattering on grain boundaries as from scattering on the wire surfaces. This indicates that efforts to mitigate size effects must increase grain size as well as surface specularity if they expect to be more than modestly successful.

The continuing industrial need for predictive simulation of feature filling spurred experiment and modeling of the superfill process itself. Consumption of adsorbed accelerator, a detrimental deviation from the surface segregation behavior that is responsible for the superfill process, was measured. Inclusion of such consumption in our Curvature Enhanced Accelerator Coverage (CEAC) model and computer code have made the filling predictions even more accurate.

This research has continued to impact industry, indicated by requests for our superfill code from Intel,

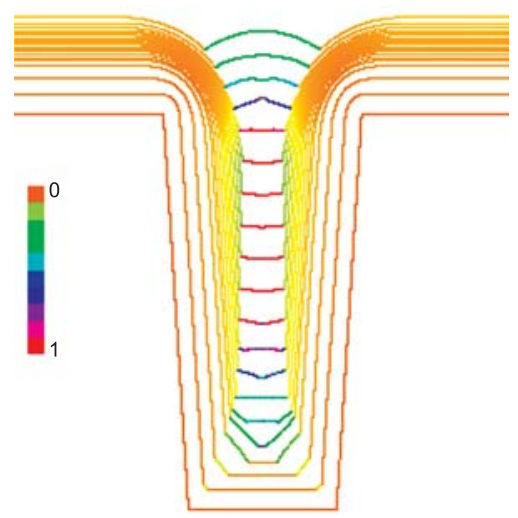

Figure 5: Simulations of superconformal feature filling now account for consumption (incorporation) of adsorbed catalyst.

Applied Materials, ST Microelectronics, and ATMI; an invited article on superfill in the IBM Journal of Research and Development; and invited presentations given at Cookson-Enthone, in addition to publications and presentations at conferences.

\section{Selected Project Publications for FY2004}

T.P. Moffat, D. Wheeler, M. Edelstein and D. Josell, "Superconformal Film Growth: Mechanism and Quantification," IBM J. Res. and Dev., in press.

D. Josell, C. Burkhard, Y. Li, Y.-W. Cheng, R.R. Keller, C.A. Witt, D. Kelley, J.E. Bonevich, B.C. Baker, T.P. Moffat, "Electrical Properties of Superfilled Sub-Micrometer Silver Metallizations," J. Appl. Phys. 96 (1), 759-768, (2004).

D. Wheeler, T.P. Moffat, G.B. McFadden, S. Coriell and D. Josell, "Influence of Catalytic Surfactant on Roughness Evolution During Film Growth," J. Electrochem. Soc. 151 (8), C538-C544, (2004).

T.P. Moffat, D. Wheeler, and D. Josell, "Electrodeposition of Copper in the SPS-PEG-Cl Additive System: I. Kinetic Measurements: Influence of SPS," J. Electrochem. Soc. 151 (4), C262-C271, (2004).

W.J. Evans, D.G. Giarikos, D. Josell, and J.W. Ziller, "Synthesis and Structure of Polymeric Networks of Silver Hexafluoroacetylacetonate Complexes of THF, Toluene, and Vinyltrimethylsilane," Inorg. Chem. 42, 8255-8261, (2003).

\section{For More Information on this Topic}

D. Josell, T.P. Moffat (Metallurgy Division, NIST); G. McFadden (Mathematical and Computational Sciences Division, NIST); R.R. Keller, Y.-W. Cheng (Materials Reliability Division, NIST); C. Witt (Cookson-Enthone); C. Burkhard, Y. Li (Clarkson University); D. Wheeler (University of Maryland); T.K. Aaltonen, M. Ritala, M. Leskelä (University of Helsinki, Finland) 


\section{Dielectric Metrology and Test Methods Supporting Embedded Passive Device Technology}

\begin{abstract}
Embedded passive devices require high dielectric constant hybrid materials consisting of filled polymers to advance miniaturization and functional performance of high-speed electronics. New metrology methods were developed to address the needs of the electronics industry. Two test methods, a Test Method for Dielectric Permittivity and Loss Tangent of Embedded Passive Materials from $100 \mathrm{MHz}$ to $12 \mathrm{GHz}$ and $a$ Test Method for Dielectric Withstanding Voltage were completed and have received wide acceptance by industry as new test methods to accelerate the development of embedded passive device technology.
\end{abstract}

\section{Jan Obrzut}

\section{Introduction}

Continuous reductions in the electrical charge required to drive logic gates and storage cells have resulted in tremendous progress in miniaturization and density of integrated circuits. However, enhanced functional performance increasingly depends on passive components such as capacitors, resistors, and inductors, where the dielectric permittivity and impedance characteristics of the material control spatial dimensions, time scale, speed, shapes and amplitude of electronic signals. Passive devices have not shrunk in size as rapidly as active devices and occupy an increasingly larger area and mass fraction of electronic subassemblies.

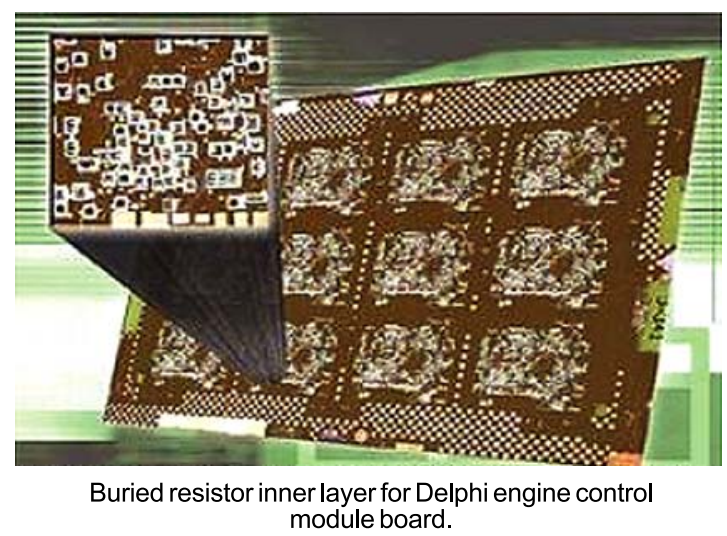

Significant advantages arise if passive devices are integrated directly into the circuit board as embedded passive devices rather than discretely attached with automated assembly. These include much thinner and sleeker electronics such as cell phones, decreased manufacturing costs, better electrical performance, and enhanced design flexibility. Companies including Gould, Shipley, Ohmega, MacDermid, DuPont, Motorola, Oak-Mitsui, 3M, and Sanmia have devoted millions of dollars to advance this technology in an effort that represents a huge transition for the electronics industry.

High dielectric constant hybrid materials consisting of filled polymers are essential for advancing miniaturization and functional performance. There are significant challenges in developing new thin-film materials that require enhanced electrical performance characteristics, such as impedance and high dielectric constant (high-k), in order to operate at higher microwave frequencies and at increased voltage strengths. Consequently, new metrology and test methods are needed to address the specific behavior of thin film specimens and to better understand the relation between functional performance and the structural attributes of the materials.

The materials and testing requirements for Embedded Passives Technology are outlined in the National Electronics Manufacturing Initiative (NEMI) 2004 Roadmap. A need for new standard test methods for embedded materials was identified by the Association Connecting Electronic Industries (IPC), Roadmap 2005 Outlook Update, IPC Embedded Passive Device Standard Committee D-39. In partnership with industry, NIST chaired a test methods task group to communicate and collaborate with industrial partners. This year, two new test methods were accepted and will be used extensively by industry to accelerate implementation of this new technology.

\section{Dielectric Permittivity and Loss Tangent}

The development of new thin-film materials that exhibit enhanced electrical performance characteristics, such as impedance and high dielectric constant (high-k), requires a broadband measurement technique for measuring permittivity at microwave frequencies (100 MHz to $12 \mathrm{GHz}$ ). Instead of expensive, single-test microwave strip test structures, NIST forwarded a general measurement strategy of incorporating a thin passive film material into a device that is comprised of a capacitive or resistive termination in a transmission line. The technique is based on the observation and theoretical analysis of the fundamental mode propagating at high frequencies in thin film dielectrics that terminate a coaxial air-filled transmission line. The development of the method involved nearly 50 active industrial partners and was reviewed by more than 300 other parties. 


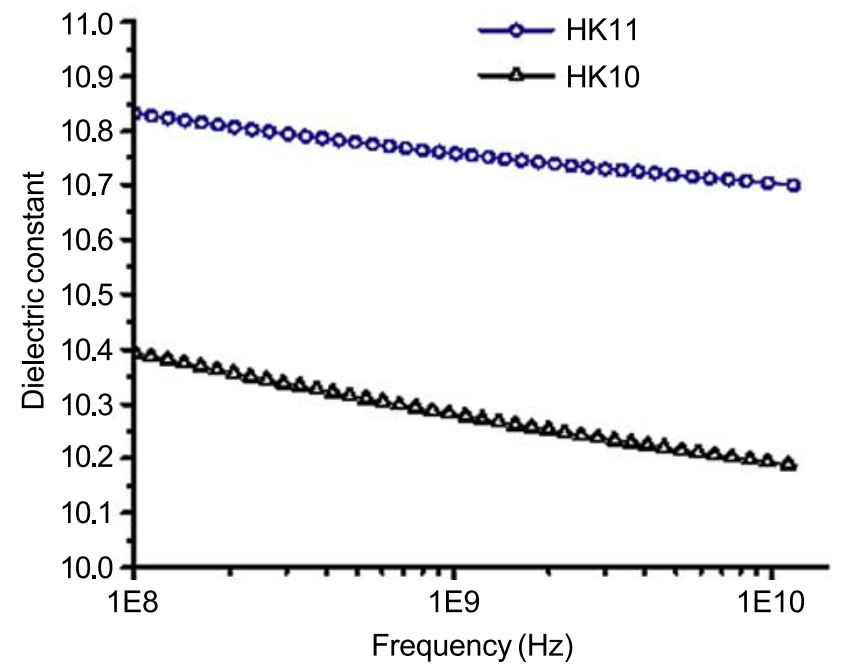

Figure 1: Dielectric constant of high-k polyimide-based composite films determined according to the Standard Test Method. (HK10 is a $25 \mu \mathrm{m}$ thick composite; HK11 is a $14 \mu \mathrm{m}$ thick composite).

An example measurement of the dielectric constant for a high-k material obtained from DuPont is shown in Figure 1. We guided the design of the test protocol and made arrangements with co-sponsoring member companies for round robin evaluation of the measurement method. The work resulted in a test document "Test Method for Dielectric Permittivity and Loss Tangent of Embedded Passive Materials from $100 \mathrm{MHz}$ to $12 \mathrm{GHz}$," which has received wide industrial acceptance and recommendation as a new standard test method.

This new capability to measure the dielectric properties at high frequencies for a wide range of dielectric permittivity values is also used to quantify dispersion, alignment, and structure in hybrid materials. For example, we demonstrated that composites of organic polymer resins filled with ferroelectric ceramics exhibit a dominant intrinsic high-frequency relaxation behavior. Such dielectric properties are beneficial in enhancing performance of processors and logic devices.

\section{Dielectric Withstanding Voltage}

The dielectric breakdown of thin film materials is also a significant issue in embedded passive devices. Due to a thin film configuration, nonlinear effects may be activated at moderate bias levels and contribute to the dielectric breakdown. A non-linear dielectric measurement methodology was developed and applied for testing passive materials at high electric fields and voltages.

NIST-developed experimental set-up and testing procedures demonstrated the unambiguous determination of the dielectric withstanding voltage of embedded passive materials. In contrast to conventional procedures, the specimen voltage and current are determined as complex quantities from the corresponding time-resolved voltage waves. The new testing procedure represents an extension compatible with the existing standard test method, but is better suited for capacitive and resistive thin film materials.

The specimen impedance and the loss tangent of the material can be determined by performing complex algebra calculations. It was found that thin-film materials for embedded passives do not exhibit a flat impedance characteristic, as is the case for conventional dielectrics, but the impedance sharply decreases with increasing voltage. The voltage withstanding condition may be attributed to a voltage range where the impedance characteristic remains insignificantly affected by the applied voltage. An equivalent impedance comparison indicates that high-k organic composites can withstand only a small fraction of the conventional dielectric withstanding voltage.

The NIST-developed procedure of recording and analyzing waveforms also allows the evaluation of specific characteristics of materials that cannot be readily evaluated with conventional techniques. This measurement procedure is especially suitable for detecting and analyzing non-linear dielectric effects that can result from polarization reversal and rectifying barriers. Such effects may appear at relatively low voltages in nano-sized interfaces, composites, and sub-micron thin dielectric films that are of interest to emerging technologies.

\section{For More Information on This Topic}

A. Anopchenko, K. Kano (Polymers Division, NIST); D. McGregor, G.S. Cox, J. Felten (DuPont); D. Fritz (MacDermid); T. Bergstresser (Gould Electronics); K. Fjeldsted (Electro Scientific Industries); R. Crosswell (Motorola); C. Vanderpan (UL); R. Whitehouse (Sanmina-SCI)

J. Obrzut and A. Anopchenko, "Input Impedance of a Coaxial Line Terminated with a Complex Gap Capacitance - Numerical and Experimental Analysis," IEEE Transactions on Instrumentation and Measurement, 53 (4), August (2004).

J. Obrzut and K. Kano, "Measurement of Complex Impedance at High AC Voltages using Waveforms," IEEE, IMTC/2004 3, 1994-1997 (2004).

J. Obrzut, A. Anopchenko, K. Kano, and H. Wang, "High Frequency Loss Mechanism in Polymers Filled with Dielectric Modifiers," Mat Res. Symp. Proc. 783, B3.5-8 (2003).

J. Obrzut and A. Anopchenko, "High frequency input impedance characterization of dielectric films for power-ground planes," IEEE Transactions on Instrumentation and Measurement 52, 1120-1124 (2003). 


\section{Friction Scaling Artifact}

Nanofrictional forces are an important factor in the design of nanodevices and assemblies of nanoparticles. Literature data on nanofriction, however, exhibit many discrepancies and have a wide range of scatter. In a series of well-controlled experiments, we have discovered that a significant portion of this discrepancy may actually be attributed to an unintended scratching of the surface by the sharp tips used in making the measurements themselves. Deviation of tip shape from the "ideal" spherical shape is another contributor to the disagreements found among the literature data. This work sheds important insight into the nature of nanoscale friction measurement.

\section{Z. Charles Ying and Stephen M. Hsu}

\begin{abstract}
$\mathrm{A}^{\mathrm{c}}$ ccurate measurement of nanofriction is emerging as one of the critical issues in nanotechnology. Manipulation of molecules, clusters, and nanoparticles across surfaces to form a functional entity relies on the ability to overcome the resistance of the particles to motion across the surface. Further, reliable operation of micro- and nano-devices often depends on an accurate estimate of lateral loading. Literature data on nanofriction, however, have shown wide ranges of values depending on the length scale of the device. Silicon friction, e.g., shows an order of magnitude variation from 0.03 to 0.25 . Such results have led to speculations that there might be an intrinsic scaling effect such that different friction levels would occur across the nano-, micro-, and macro-scales. If proven true, well established friction laws would be invalidated for nanomaterials.
\end{abstract}

Given the broad technological significance of this issue, we have undertaken a detailed investigation of the nature of this discrepancy. Friction measurements between non-adhering surfaces were carried out in both elastic and plastic deformation regions. Diamond tips of various sizes were used to measure nanofriction forces on well-characterized substrates such as single crystal silicon, silicon dioxide, and calcium fluoride. Experiments were performed using a specially designed multiscale friction tester developed jointly by NIST and Hysitron Incorporated based on a capacitance probe force transducer. In each experiment, the environment was carefully controlled, and the tip penetration into the substrate during sliding was continuously measured.

The size and shape of each tip were characterized using a newly developed "replica technique." Tips were imprinted into a soft material, and the imprints were scanned by AFM and digitally inverted to
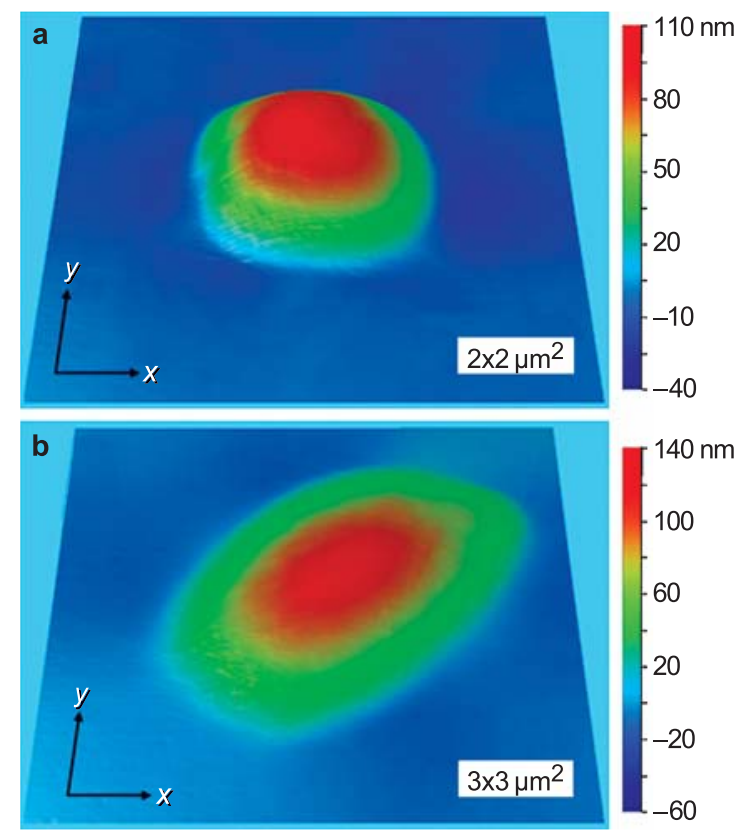

Figure 1: 3D profiles, obtained using the replica technique, of the tips used in the friction measurements.

"reconstruct" the tip in three-dimensional details. Figure 1 shows images of two of the diamond tips used in the experiments. The tip shown in Figure 1(a) is spherical in shape with a $1.2 \mu \mathrm{m}$ tip radius while Figure 1(b) shows an ellipsoidal shape with nominal radius of $4 \mu \mathrm{m}$. The detailed dimensional data are crucial in determining the apparent contact areas and isolating the influence of surface forces.

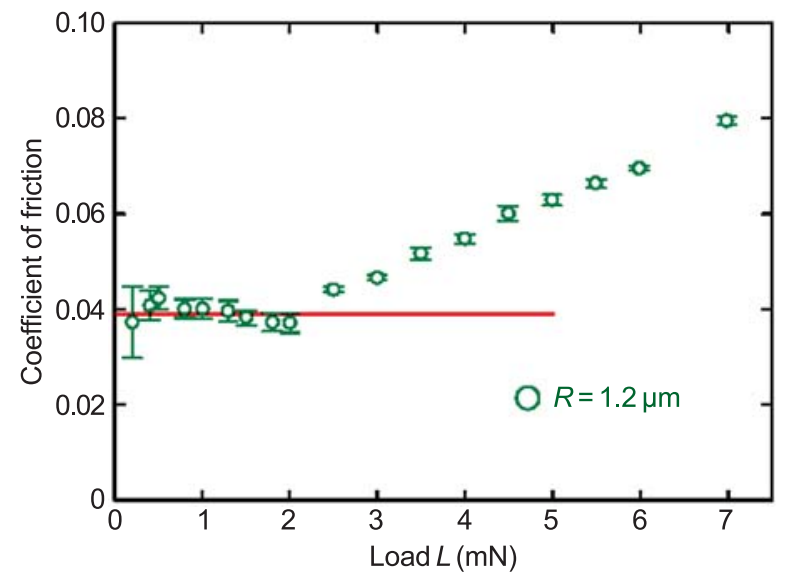

Figure 2: Coefficient of friction between Si (100) surface and a diamond tip with radius $\mathrm{R}=1.2 \mu \mathrm{m}$, as a function of mechanical loading force.

Figure 2 shows the coefficient of friction (COF) between a Si (100) surface and a diamond tip with tip radius $R=1.2 \mu \mathrm{m}$. 


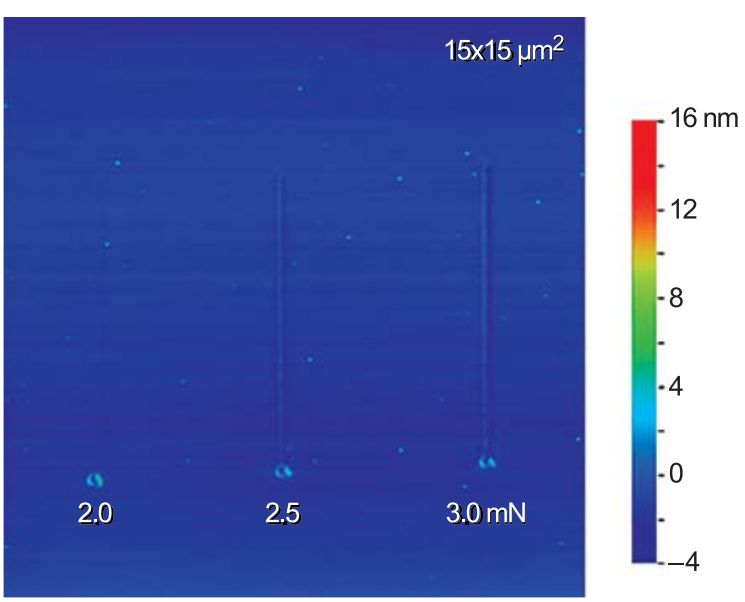

Figure 3: Image of the silicon surface after friction measurements, showing scratches at $2.5 \mathrm{mN}$ and higher load.

Two distinct regions can be seen. Initially, the COF is constant up to a load of $2.0 \mathrm{mN}$. For loads above $2.5 \mathrm{mN}$, the COF increases with load. This friction transition is contrary to Amoton's law. Subsequent investigation revealed that this change in COF is caused by unintended plowing of the tip into the substrate. Atomic force microscopy, using a sharp tip on the surface, suggests that the COF increase coincides with the first appearance of plastic grooving. In the case of silicon, a groove $5 \AA$ deep and $2000 \AA$ wide was detected on the surface at a load of $2.5 \mathrm{mN}$ (Figure 3).

This observation was repeated for different tips and different substrates. At higher loads, deeper and wider grooves were observed. These results suggested that unintended scratching of the surfaces by the sharp tips used in the friction measurements contributed to the discrepancies among friction data in the literature.

The initiation of plastic grooving can be quantified in terms of contact pressure. By determining the apparent contact area using the digitally inverted tip method, the transition contact pressures could be calculated for all of the observed cases. All the results corresponded to $12 \mathrm{GPa}$ which is approximately the hardness of silicon.

\section{Elastic Plowing}

Figure 4 shows the friction data for three tips in the elastic region (no plastic grooving). The data for the two spherical tips with radii $R=0.5 \mu \mathrm{m}$ and $1.2 \mu \mathrm{m}$ exhibit a common value for the COF. However, a higher friction value was observed for the $4 \mu \mathrm{m}$ radius tip.

To explain this discrepancy, we tested the various contact mechanics models which require the accurate determination of the real contact areas. Considering a tip plowing through an elastic substrate, the real area of contact is the projected cross-section area of the tip in the direction of sliding. This can be determined from the tip geometry and "penetration depths" across the sliding path. Numerical integration of actual tip size and shape over the sliding path is required. For the hypothesis of "elastic plowing," the calculation shows that the friction force in this regime should be proportional to the projected cross-sectional area in the direction of sliding. Figure 5 confirms this hypothesis for all three tips.

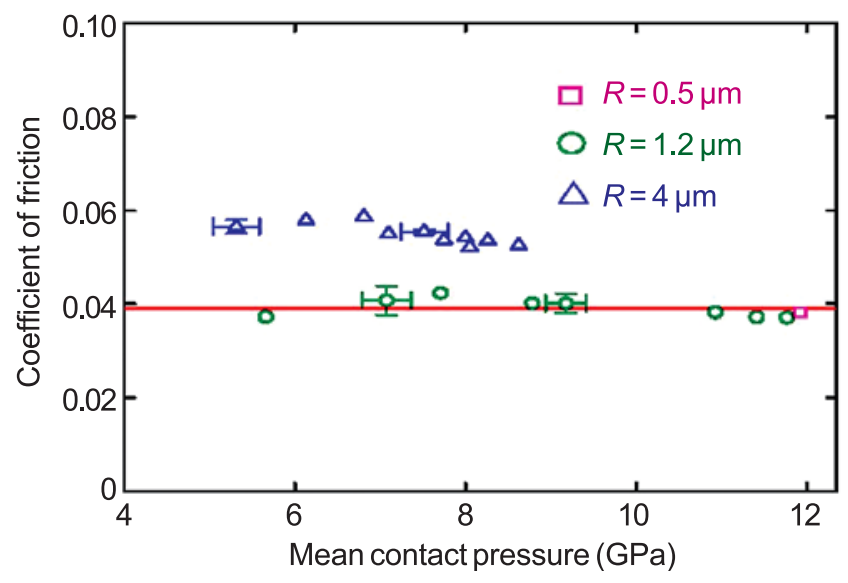

Figure 4: Coefficient of friction between Si (100) surface and three diamond tips used in the experiments, as a function of mean contact pressure.

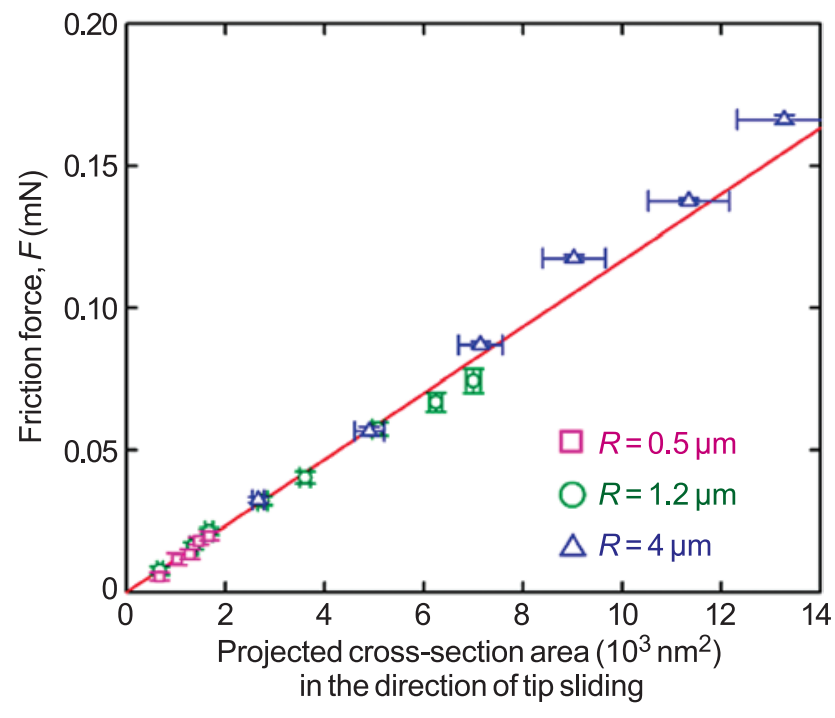

Figure 5: Friction force as a function of projected cross-section area in the direction of tip sliding.

This work shows clearly that nanoscale friction, in practice, is a complex result of both elastic and inelastic strains and deformations, and accurate nanofriction measurement will require careful accounting of all the forces contributing to the phenomenon. The methodology established in this work provides a substantial step towards the data and understanding needed by industry.

\section{For More Information on this Topic}

Z.C. Ying, S.M. Hsu (Ceramics Division, NIST) 


\section{Grand Challenges in Nanomagnetics: High Coercivity FePt Alloys for Future Perpendicular Magnetic Data Storage}

The magnetic data storage industry is seeking to sustain the 30-year trend of exponentially increasing storage density. This must be achieved at the same time as maintaining the stability of the recorded data. The Metallurgy Division at NIST is collaborating with Seagate Technology to develop the processes required to produce patterned media of high-magnetic coercivity that will meet this challenge.

Jonathan J. Mallett, Thomas P. Moffat, and William F. Egelhoff, Jr.

$\mathrm{C}$ onventional magnetic data storage media are based on granular films in which many fine grains are used to define each magnetic bit. The minimum number of grains per bit is limited by the irregularity of the shape and spacing of the grains, which produces irregular boundaries between bits. This irregularity results in noise in the read signal and can only be constrained at an acceptable level by maintaining a fixed minimum number of grains per bit. The natural approach to increasing data storage density has been to reduce the grain size, while maintaining the same number of grains per bit. The limit to this approach occurs when the magnetic energy barrier to the reversal of magnetization of each grain approaches the energy of random thermal fluctuations. At this point, the medium is no longer stable against spontaneous magnetization reversal and consequential data loss.

The grain size at which this limit occurs depends on the magnetocrystalline anisotropy energy of the

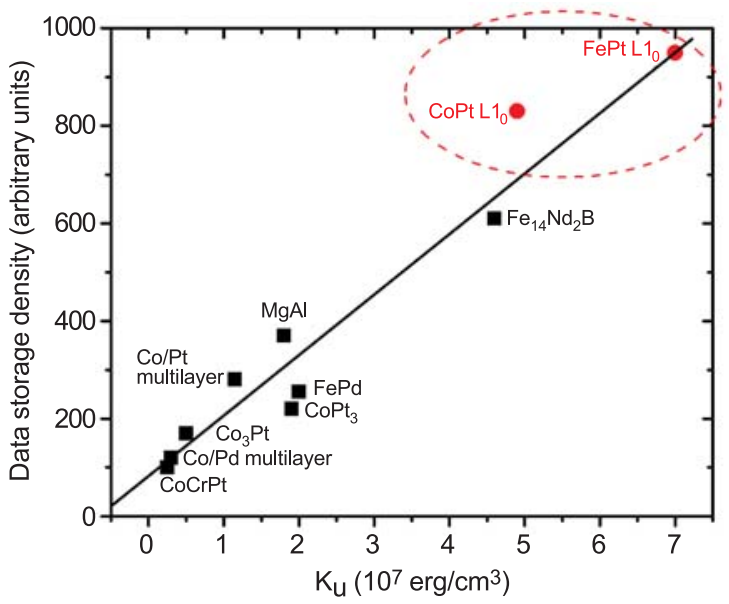

Figure 1: The dependence of storage density on magnetocrystalline anisotropy energy. The most promising materials are FePt and CoPt. magnetic material. FePt in its $\mathrm{L}_{0}$ phase has a sufficiently large anisotropy energy to allow magnetically stable grains of $5 \mathrm{~nm}$ diameter (Figure 1). Furthermore, FePt deposited by a variety of means typically forms grains approaching this size. Unfortunately, the annealing treatment required to transform the as-deposited A1-structured FePt to the desirable $\mathrm{L} 1_{0}$ phase is widely found to result in an increase in grain size to $100 \mathrm{~nm}$. Current efforts at NIST focus on developing a method of electrochemically depositing FePt into a regular patterned template, circumventing the problem of grain growth. Simultaneously, the imposed regularity of the magnetic cells opens the possibility of addressing single cells (i.e., of using one "grain" per bit). The estimated achievable density from such an approach is 7 Tbits per square inch, which is 100 times higher than current storage densities. Electrodeposition is an obvious choice for deposition into high aspect ratio templates, since it does not suffer from the shadowing effect that characterizes vacuum deposition techniques.

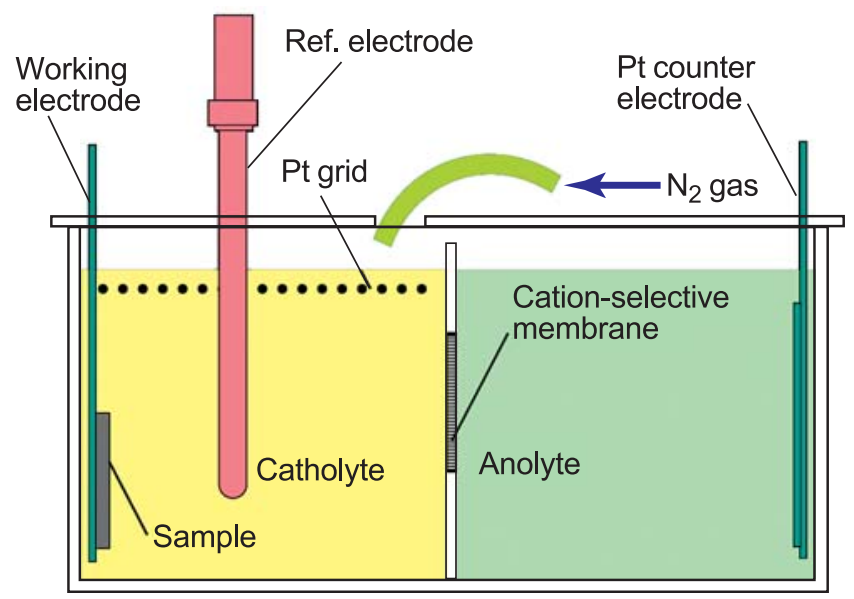

Figure 2: The double cell, designed to minimize the concentration of $\mathrm{Fe}^{3+}$ and dissolved $\mathrm{O}_{2}$ in the FePt plating solution.

Electrodeposition of FePt from aqueous solutions presents many challenges. The double cell shown in Figure 2 has been developed to address the problem of the instability of $\mathrm{Fe}^{2+}$ solutions, which readily oxidize to produce insoluble $\mathrm{Fe}(\mathrm{OH})_{3}$. The plating solution, containing $\mathrm{FeCl}_{2}$ and $\mathrm{PtCl}_{4}$ (left) is separated from the anode by a cation selective membrane, preventing the oxidation of $\mathrm{Fe}^{2+}$ to $\mathrm{Fe}^{3+}$ that would otherwise occur at the anode. $\mathrm{Fe}^{3+}$ formation by oxidation with air is also minimized by blanketing the cell in nitrogen, and residual $\mathrm{Fe}^{3+}$ is reduced to $\mathrm{Fe}^{2+}$ by an auxiliary platinum grid electrode. 


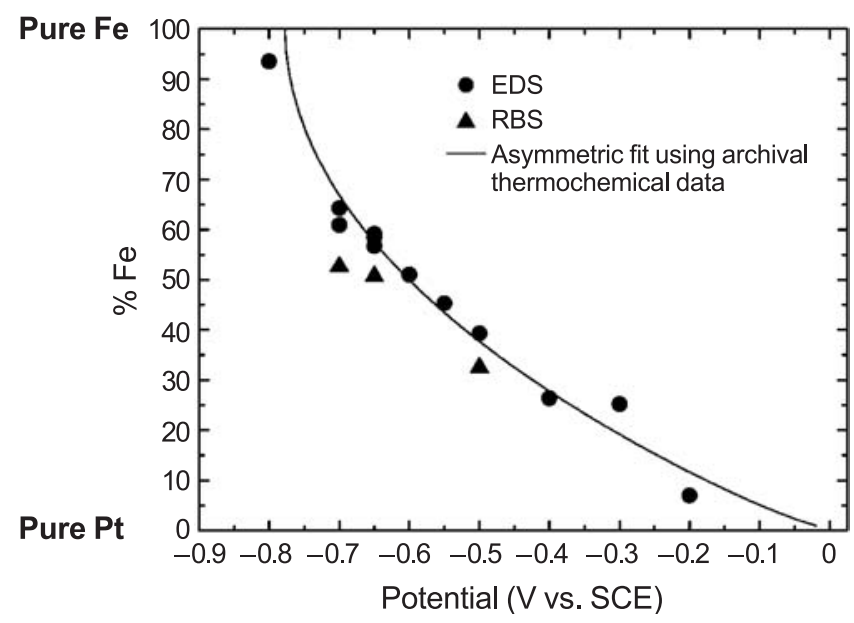

Figure 3: The theoretical and experimental dependence of composition on the applied potential.

The alloy composition of $300 \mathrm{~nm}$ thick films deposited from this solution was found to depend mainly on the applied potential and to be insensitive to the concentration of the solution components. A thermodynamic regular solution model was used to describe the dependence of film composition on applied potential. The comparison of theory to experimental data can be seen in Figure 3.

The deposited films were found to be remarkably smooth, with RMS roughness values less than $5 \mathrm{~nm}$ for micron-thick deposits. This was surprising given the large platinum overpotential (supersaturation), which usually results in growth instabilities and consequent roughening.

The control of crystal orientation is an essential consideration for high-density recording applications. A transition in the recording industry is currently in progress to perpendicular media, in which the bits are magnetized perpendicular to the plane of the medium. Maximum advantage can be derived from $\mathrm{L} 1_{0} \mathrm{FePt}$ as a perpendicular medium when it is correctly aligned with its magnetic easy-axis perpendicular to the substrate plane. A careful choice of substrate and annealing parameters is required to recrystallize the as-deposited random fcc alloy to appropriately oriented $\mathrm{L} 1_{0}$.
(001)

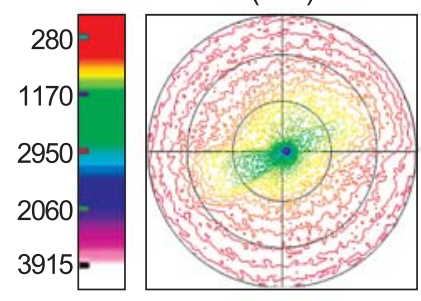

(110)

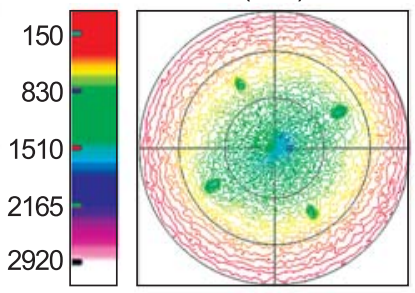

Figure 4: X-ray pole figures showing favorably oriented FePt $L 1_{0}$.
FePt electrodeposited onto a $\mathrm{Cu}(001)$ substrate has recently shown great promise. X-ray diffraction revealed the transition to $\mathrm{L} 1_{0}$ upon annealing, and $\mathrm{X}$-ray pole figures indicated favorable orientations. Figure 4 shows FePt $\mathrm{L}_{0}$ (001) and (110) pole figures. The (001) figure indicates perpendicular orientation of the magnetic easy-axis (the c-axis), while the (110) figure indicates an in-plane texture. The in-plane texture is an added advantage, as it results in a narrower switching field distribution.

Copper additions to the alloy have been found to lower the $\mathrm{A} 1 / \mathrm{L1}_{0}$ phase transition temperature by up to $90{ }^{\circ} \mathrm{C}$. It may be speculated that interdiffusion of copper from the substrate during the anneal allowed the recrystallization to proceed from the interface with the substrate. It is likely that this would allow the film to replicate the orientation of the substrate.

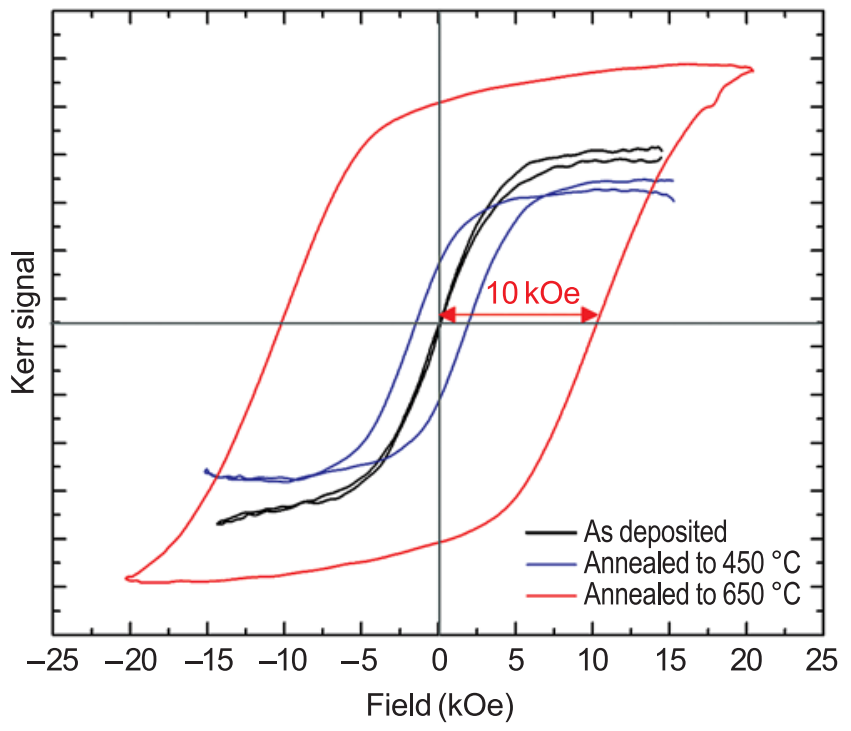

Figure 5: Magnetic hysteresis measurements showing a $10 \mathrm{kOe}$ coercivity.

Magnetic hysteresis measurements performed using a Kerr magnetometer are shown in Figure 5. The magnetic coercivity of $10 \mathrm{kOe}$ is comparable to figures quoted in the literature for vacuum-deposited FePt. It is believed to be the highest value reported for an electrodeposited film.

Current efforts focus on controlling the interdiffusion between substrate and film to minimize loss of magnetization in the alloy, and on moving from planar films to through-mask deposited arrays of FePt pillars.

\section{For More Information on this Topic}

W.F. Egelhoff, Jr. (Metallurgy Division, NIST); E.B. Svedberg (Seagate Research, Pittsburgh) 


\section{Breakthroughs in Magnetic Refrigeration}

In the past 10 to 15 years, there has been a substantial effort to find alternative cooling technologies to vapor compression systems. This has been driven largely by the need to reduce the high energy costs of present systems along with their use of harmful CFC refrigerants. Magnetic refrigeration technology is one such alternative. In principal, because it is a reversible process, magnetic cooling should be more efficient than the competing irreversible vapor compression systems. However, magnetic cooling technology has historically been relegated to cryogenic, high-magnetic field applications since efficiency was so poor at higher temperatures. In 2004, we made progress in identifying materials with greatly improved performance close to room temperature. For U.S. industry to take advantage of these new materials, their behavior needs to be understood and methods of proper measurement determined. We are providing the metrology to do that.

\section{Robert Shull, Virgil Provenzano, and Alexander Shapiro}

\footnotetext{
I
} n 1997 a large ("giant") magnetocaloric effect was reported in the $\mathrm{Gd}_{5} \mathrm{Ge}_{2} \mathrm{Si}_{2}$ compound between $270 \mathrm{~K}$ and $300 \mathrm{~K}$, thereby generating a great deal of interest as a potential near-room temperature magnetic refrigerant. Unfortunately, large hysteretic losses occur in the same temperature range where this compound exhibits its large magnetocaloric effect, making the magnetic refrigeration with the material inefficient.

In Nature [Vol. 429, p. 853 (2004)], we reported on the discovery of an effective and straightforward method for greatly reducing (by more than 90 percent) the large hysteresis losses in $\mathrm{Gd}_{5} \mathrm{Ge}_{2} \mathrm{Si}_{2}$. This is accomplished by alloying the compound with a small amount of iron, which has the additional benefit of shifting the magnetic entropy change $\left(\Delta \mathrm{S}_{\mathrm{m}}\right)$ peak to higher temperature, from $275 \mathrm{~K}$ to $305 \mathrm{~K}$, and broadening its width. Although the addition of Fe yields comparable refrigerant capacity (RC) values, a greater resultant value is obtained for the Fe-containing alloy when the hysteresis losses are subtracted. The Fe-containing alloy is clearly better for near-room temperature magnetic refrigeration applications.

Figure 1 shows the magnetization (M) $v s$. applied magnetic field $(\mathrm{H})$ data measured in the important temperature range of peak magnetocaloric effects

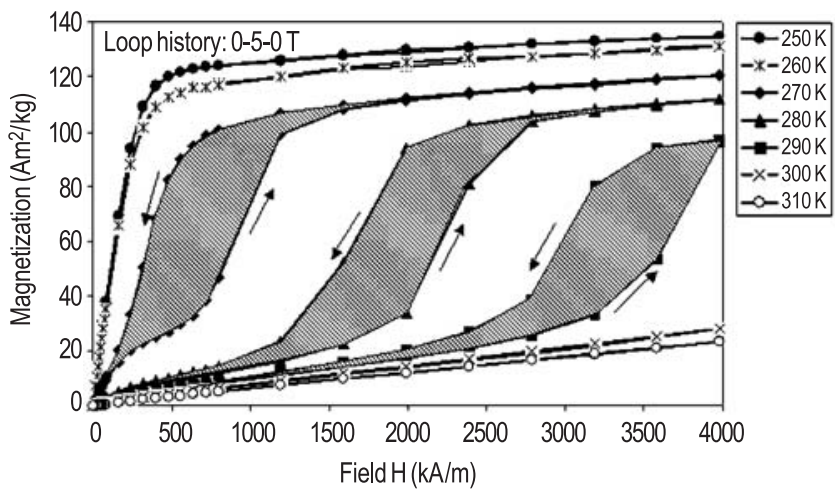

Figure 1: Magnetic hysteresis measurements showing a field-induced ferromagnetic transition and large hysteresis in the "giant magnetocaloric effect" $\mathrm{Gd}_{5} \mathrm{Ge}_{2} \mathrm{Si}_{2}$ compound.

for the $\mathrm{Gd}_{5} \mathrm{Ge}_{2} \mathrm{Si}_{2}$ compound. In this material, the enhanced values of the magnetocaloric effect have been ascribed to the existence of a field-induced crystallographic phase change in the material from a low-field paramagnetic structure to a high-field ferromagnetic crystal structure. As a consequence of this field-induced phase change, there is hysteresis in its transformation, shown by the shaded areas enclosed within the field cycles, corresponding to the cost in energy to make one cycle. The magnitude must be assessed when determining whether a particular refrigerant is useful in a given application.

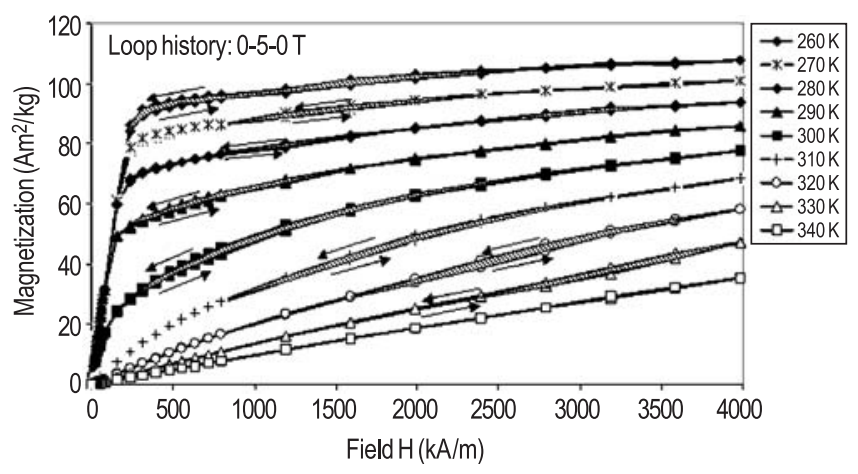

Figure 2: Magnetic hysteresis measurements showing no field-induced ferromagnetic transition and nearly zero hysteresis in the $\mathrm{Gd}_{5} \mathrm{Ge}_{1.9} \mathrm{Si}_{2} \mathrm{Fe}_{0.1}$ alloy.

We have found that by adding a small amount of Fe to the $\mathrm{Gd}_{5} \mathrm{Ge}_{2} \mathrm{Si}_{2}$ compound, the hysteresis may be significantly reduced. This effect is shown in the $\mathrm{M} v s . \mathrm{H}$ data for this alloy in the important temperature range of potential use in Figure 2, where the shaded hysteresis regions are nearly zero. 


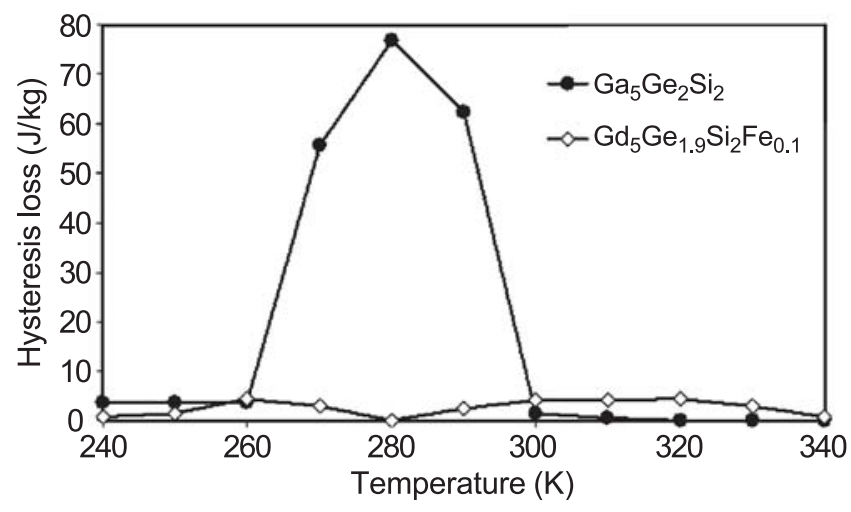

Figure 3: Hysteresis energy loss as a function of temperature for the $\mathrm{Gd}_{5} \mathrm{Ge}_{2} \mathrm{Si}_{2}$ and $\mathrm{Gd}_{5} \mathrm{Ge}_{1.9} \mathrm{Si}_{2} \mathrm{Fe}_{0.1}$ alloys.

A magnetic field is alternately applied and removed from a refrigerant during a single cooling cycle in a magnetic refrigerator, thus any magnetic hysteresis will reduce the usefulness of a material. Figure 3 shows exactly how much of a difference in hysteresis there is between the $\mathrm{Gd}_{5} \mathrm{Ge}_{2} \mathrm{Si}_{2}$ and $\mathrm{Gd}_{5} \mathrm{Ge}_{1.9} \mathrm{Si}_{2} \mathrm{Fe}_{0.1}$ alloys.

The reduced hysteresis in the quaternary alloy is due to a lack of field-induced paramagnetic-to-ferromagnetic crystallographic phase change. This change in behavior is caused by the Fe combining with primarily the $\mathrm{Si}$ in the ternary compound to form an Fe-rich secondary phase, thereby reducing the Si content in the majority Gd-Ge-Si phase. As a consequence, the thermodynamics of the Gd-Ge-Si alloy were changed from that of the precursor $\mathrm{Gd}_{5} \mathrm{Ge}_{2} \mathrm{Si}_{2}$ compound.

The magnetic entropy change $\left(\Delta \mathrm{S}_{\mathrm{m}}\right)$ for a $5 \mathrm{~T}$ applied field as a function of temperature for both alloys is shown in Figure 4. The computed refrigerant capacities (RC) are indicated on the figures and shown by the shaded areas. Compared to $\mathrm{Gd}_{5} \mathrm{Ge}_{2} \mathrm{Si}_{2}$, the iron-containing compound exhibits a smaller, but broader $\Delta \mathrm{S}_{\mathrm{m}}$ peak, and a $\Delta \mathrm{S}_{\mathrm{m}}$ peak at a higher temperature. Purely on this basis, $\mathrm{Gd}_{5} \mathrm{Ge}_{2} \mathrm{Si}_{2}$ is the superior material, but the Fe-containing alloy might be attractive for higher temperature applications due to its higher temperature peak.

One method to account for the hysteresis losses of each alloy is to simply subtract it from the corresponding refrigerant capacity value. Subtraction of the average hysteresis in the temperature range of the $\Delta \mathrm{S}_{\mathrm{m}}$ peak for each alloy $(\sim 65 \mathrm{~J} / \mathrm{kg}$ and $\sim 4 \mathrm{~J} / \mathrm{kg}$, respectively, for the ternary and quaternary alloys) from the corresponding refrigerant capacity values yielded approximate values of $240 \mathrm{~J} / \mathrm{kg}$ for the Fe-free alloy and $355 \mathrm{~J} / \mathrm{kg}$ for the Fe-containing alloy. Consequently, when the hysteresis losses are taken into account, it is clear that the $\mathrm{Gd}_{5} \mathrm{Ge}_{1.9} \mathrm{Si}_{2} \mathrm{Fe}_{0.1}$ alloy is a much
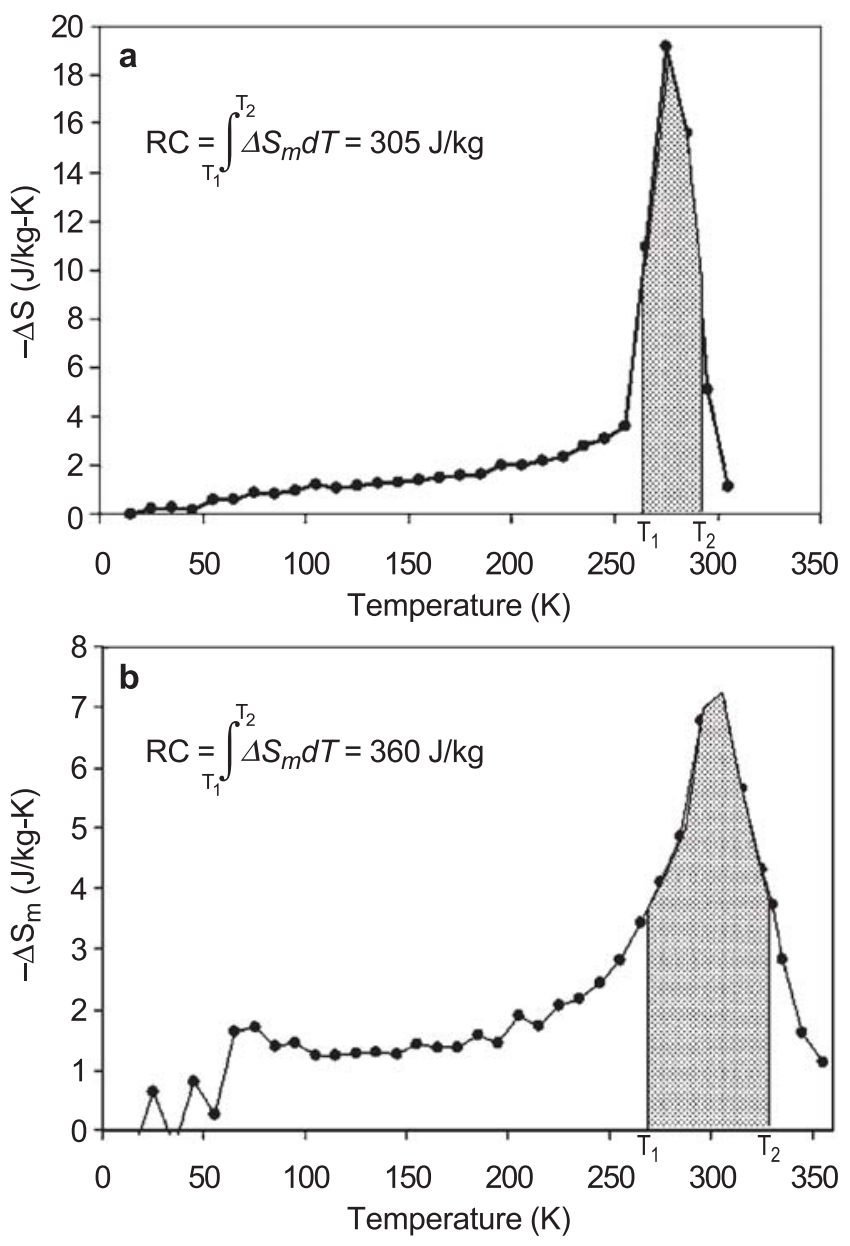

Figure 4: Computed $\Delta S_{m}[$ for $\Delta H=3980 \mathrm{kA} / \mathrm{m}(5 \mathrm{~T})]$, normalized with respect to sample mass, of the $\mathrm{Gd}_{5} \mathrm{Ge}_{2} \mathrm{Si}_{2}$ compound (top) and of the $\mathrm{Gd}_{5} \mathrm{Ge}_{1.9} \mathrm{Si}_{2} \mathrm{Fe}_{0.1}$ alloy (bottom), plotted as a function of temperature showing the presence of peaks centered near $270 \mathrm{~K}$ and $305 \mathrm{~K}$ respectively for $\mathrm{Gd}_{5} \mathrm{Ge}_{2} \mathrm{Si}_{2}$ and $\mathrm{Gd}_{5} \mathrm{Ge}_{1.9} \mathrm{Si}_{2} \mathrm{Fe}_{0.1}$.

better magnetic refrigerant than the $\mathrm{Gd}_{5} \mathrm{Ge}_{2} \mathrm{Si}_{2}$ compound despite its lower $\Delta \mathrm{S}_{\mathrm{m}}$ peak value. Most prior evaluations of magnetic refrigerants have disregarded the material hysteresis losses, and in some materials these losses can be quite large; this example shows the weakness in that approach.

"The results are pretty amazing," states Michael DiPirro [Science News 165, 405 (2004)], a cryogenic engineer at the NASA Goddard Space Flight Center in Greenbelt, Maryland.

\section{For More Information on this Topic}

Virgil Provenzano, Robert D. Shull, and Alexander J. Shapiro, "Reduction of hysteresis losses in the magnetic refrigerant $\mathrm{Gd}_{5} \mathrm{Ge}_{2} \mathrm{Si}_{2}$ by the addition of Iron," Nature 429, 853 (2004). 


\section{Quantitative Nanomechanical Properties}

We are developing AFM-based metrology for rapid, nondestructive measurement of mechanical properties with true nanoscale spatial resolution. Atomic force acoustic microscopy methods enable elastic-modulus measurements at either a single point or as a map of local property variations. Complementary information obtained with scanning electron microscopy provides insight into structure-property relations and helps to interpret nanoscale contact-mechanics behavior. The information obtained furthers our understanding of the nanomechanical properties of surfaces, thin films, and nanoscale structures.

\section{Donna C. Hurley}

$\mathrm{N}$ ew measurement solutions are required to address the rapidly burgeoning field of nanotechnology. In particular, information about mechanical properties on the nanoscale is needed. Knowledge of properties like elastic modulus and interfacial quality (defects, strain, adhesion, etc.) is critical to successful development of new films and nanoscale assemblies. Such information could also assess integrity or reliability in applications from microelectronics to biotechnology. Existing methods for mechanical-property measurements have drawbacks: they are destructive, limited to specialized test specimens, or not quantitative. Instrumented or "nano-" indentation (NI), a current industry workhorse, will have limited value as scales shrink well below $1 \mu \mathrm{m}$, and softer materials are more frequently used.

To meet this need, we are developing tools that exploit the spatial resolution of atomic force microscopy (AFM). Our approach, called atomic force acoustic microscopy (AFAM), involves the vibrational resonance of an AFM cantilever when its tip is in contact with a sample. By comparing the cantilever's contact-resonance frequencies for an unknown material to those for a reference sample with known properties, the indentation modulus $M$ of the unknown material can be determined. [For an isotropic material $M=E /\left(1-v^{2}\right)$, where $E$ is Young's modulus and $v$ is Poisson's ratio.] The small tip radius $(\sim 5-50 \mathrm{~nm})$ means that we can obtain in-situ elastic stiffness images with nanoscale spatial resolution.

In FY04, we extended our quantitative AFAM techniques in a variety of ways. In one effort, the effect of film thickness on measurement accuracy was investigated. We measured $M$ for three nickel (Ni) films approximately 50, 200 and $800 \mathrm{~nm}$ thick. The values of $M$ ranged from $220 \mathrm{GPa}$ to $223 \mathrm{GPa}$, significantly lower than that expected for bulk polycrystalline Ni. Scanning electron microscopy (SEM) revealed that the films were nanocrystalline (grain diameter $<30 \mathrm{~nm}$ ). The observed reduction in $M$ may be attributed to an increased volume fraction of grain boundaries in the nanocrystalline films. More importantly, the average values of $M$ for all three films were the same within measurement uncertainty $(\sim 10 \%)$. Thus the AFAM results were not influenced by the elastic properties of the silicon substrate, even for a $50 \mathrm{~nm}$ film. This behavior is due to the fact that the AFAM stress field extends less than $100 \mathrm{~nm}$ into the sample and decreases rapidly with depth due to the small applied static loads $(0.3-3 \mu \mathrm{N})$ and small radius of contact $(5-25 \mathrm{~nm})$. Our result contrasts sharply with nanoindentation, in which substrate properties must be included to accurately measure submicrometer films.

The elastic properties of the $800 \mathrm{~nm}$ Ni film were also measured using NI, microtensile testing, and surface acoustic wave spectroscopy (SAWS). Both AFAM and NI measure the film's out-of-plane indentation modulus. The results were in excellent agreement, validating AFAM as a quantitative method in spite of its relative newness. Microtensile testing values for the in-plane Young's modulus of the film were not consistent with the AFAM and NI results if the film was assumed to be elastically isotropic. The apparently contradictory results were reconciled by use of a transversely anisotropic model for the film's elastic properties. This model is consistent with the strong $<111>$ film texture observed by $x$-ray diffraction. When analyzed with the same model, the SAWS results indicated that the film density was only slightly lower $(<5 \%)$ than the bulk value. These results illustrate a relatively straightforward way to interpret mechanicalproperty measurements of thin films that is based on a more physically realistic model than the simple assumption of elastic isotropy.

Another effort investigated the effects of relative humidity (RH) on AFAM measurements. AFAM contact

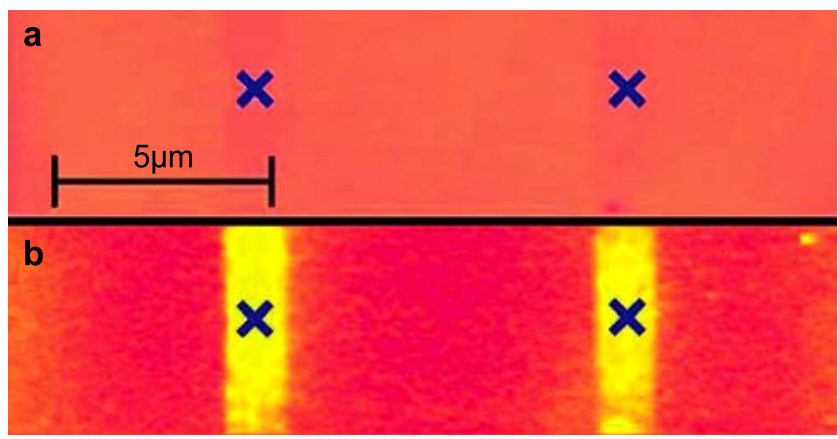

Figure 1: a) AFM tapping-mode topography and b) AFAM relative-stiffness images of $S i$ with $n$-octyldimethylchlorosilane SAM. 
stiffness measurements may be affected by a variable water layer between the tip and sample, at least in some cases. By refining our data analysis methods to include the effects of such a layer, apparent correlations between the measured values of $M$ and the ambient RH were eliminated. This year we also developed a RH-controlled AFM chamber in order to systematically study these effects. Samples for the experiments were provided by the Polymers Division and consisted of patterned self-assembled monolayers (SAM) of n-octyldimethylchloro-silane on Si substrates.

Through controlled ultraviolet-ozone exposure, the relative hydrophobic/hydrophilic nature of the SAM was adjusted in different sample regions. Figure 1 shows an AFM topography image and an AFAM relative-stiffness image for a $\mathrm{SAM} / \mathrm{Si}$ sample. The SAM stripes are virtually invisible in the topography image, even at very high resolution (10 $\mathrm{nm}$ full scale height). However, the AFAM image clearly reveals the hydrophobic SAM stripes. The regions covered by the SAM appear more compliant (lower contact stiffness) due to AFAM's sensitivity to local variations in the tip-sample adhesion. We are currently performing further experiments on similar samples in order to quantify how humidity and adhesion effects can be distinguished from true mechanical-property variations.

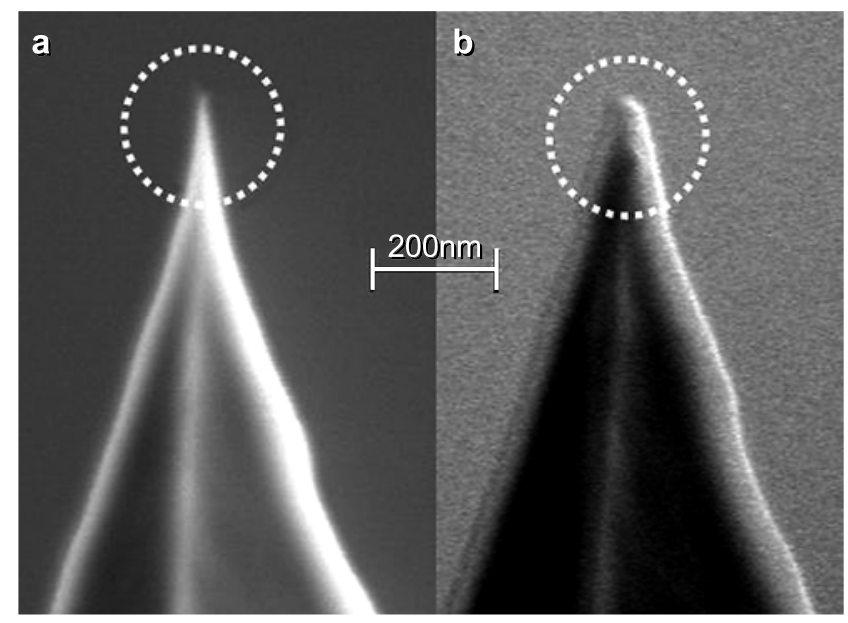

Figure 2: SEM images of AFM cantilever tip a) before use and b) after repeated AFAM contact experiments. The circled regions indicate the tip wear that occurred through use.

AFAM contact-resonance frequencies depend not only on elastic properties, but also on the value of the tip radius $R$. Thus knowledge of $R$ and how it changes over time is essential for accurate measurements of elastic properties. To address this issue, we performed AFAM experiments with different AFM cantilevers on a sample with known elastic properties. The first measurements were done at relatively low static loads; the load was then successively increased up to several micronewtons to try to break and/or plastically deform the tip. High-magnification SEM images were obtained before and after each AFAM measurement. As can be seen in Figure 2, $R$ increased with use, indicating tip wear. Values of $R$ measured from the SEM images were compared to the values obtained from AFAM data using a Hertzian contact-mechanics model. The AFAM values of $R$ were consistently smaller than the SEM values. Further data analysis and additional experiments are planned to clarify this issue. The knowledge gained in this way will help us to refine our understanding of AFAM contact mechanics, beyond the Hertz approximation, in order to improve measurement accuracy and repeatability.

In related work, we are performing finite-element studies of the AFM cantilever. The finite-element mesh is based on actual cantilever dimensions from SEM images and includes elastic anisotropy. The predicted free-space resonant frequencies are in excellent agreement with those observed experimentally. Work is underway to predict the vibrational behavior in contact. These results will allow us to refine our data analysis models and thus improve measurement accuracy.

The research described above involves either quantitative single-point measurements, or qualitative imaging of relative stiffness. In FY04, we worked to realize our ultimate goal of quantitative nanomechanical mapping. Critical to our success is a new frequency tracking circuit that can determine the contact-resonance frequencies at each image pixel. The circuit is based on digital signal processing architecture that enables rapid data acquisition (typically $<30 \mathrm{~min}$. for a $256 \times 256$ image). We have begun to obtain resonance-frequency images for a variety of materials. Recent enhancements to our AFM mean that we can acquire images from not only the flexural modes, but also the torsional modes of the cantilever. By combining information from flexural and torsional images, it may be possible to determine simultaneously both Young's modulus and Poisson's ratio for an isotropic material. Work in upcoming months will focus on issues related to quantitative image interpretation such as calibration procedures, cantilever selection, tip wear, and choice of contact-mechanics model. Each of these elements plays a role in attaining our goal of truly quantitative nanomechanical imaging.

\section{For More Information on this Topic}
D.C. Hurley (Materials Reliability Division, NIST) 


\section{Data and Standards Resources}

Advances in technology and the vitality of basic research have an unequivocal dependence on reliable data. Indeed, the technology transfer between research and application is facilitated and sometimes enabled by the development and accessibility of carefully designed and evaluated databases. The MSEL Ceramics Division has excelled in its leadership in data resources for crystallography, phase equilibria diagrams, and materials property data.

\section{Ronald G. Munro, Terrell A. Vanderah, and Vicky L. Karen}

The issue of reliable data has been examined vigorously in numerous studies over the last three decades. Early assessments, such as Critical Surveys of Data Sources (NBS SP 396-1, 1978), voiced concerns over the availability of data, while other studies, such as Economic Effects of Corrosion (NBS SP 511-1\&2, 1978), noted the influential role that data can perform as a component of a sound economy. More recently, defense and security concerns, Materials Research to Meet 21st Century Defense Needs (NMAB-498, 2002), have continued to stress the need for reliable data.

\begin{tabular}{|c|c|c|c|c|c|}
\hline & & $\begin{array}{l}\text { X-Ray } \\
\text { SRMs }\end{array}$ & $\begin{array}{c}\text { Crystal } \\
\text { Data }\end{array}$ & $\begin{array}{l}\text { Materials } \\
\text { Properties }\end{array}$ & $\begin{array}{l}\text { Phase } \\
\text { Equilibria }\end{array}$ \\
\hline \multirow{3}{*}{ Core NIST functions } & Metrology standards & $\bullet$ & & & \\
\hline & Metrology facilities & $\bullet$ & & & \\
\hline & Evaluation methods & & $\bullet$ & $\bullet$ & $\bullet$ \\
\hline Strategic focus areas & Information management & & $\bullet$ & $\bullet$ & $\bullet$ \\
\hline \multirow{2}{*}{ National needs } & Materials data & & $\bullet$ & $\bullet$ & $\bullet$ \\
\hline & Measurement tracebility & $\bullet$ & & & \\
\hline NIST obligation & Standard reference data & & $\bullet$ & $\bullet$ & $\bullet$ \\
\hline
\end{tabular}

Figure 1: Programmatic contributions of the Data and Standards Technology Group to the NIST mission.

The Ceramics Division has made a substantial effort to address the multiple needs for materials data, the scientific fundamentals for data evaluation methodology, and the technological issues involved in optimizing the utility of the data resources. Programmatically, Figure 1, the data and standards technology projects are well attuned to NIST's core mission and its strategic focus efforts, and there is abundant evidence that our objectives are being met with considerable success.

There are several technology transfer mechanisms that can be used for data and standards work, including traditional publications, internet distribution, software products for personal computers, and standard reference materials. The Division's most readily visible mechanism for data technology transfer is provided by the Ceramics WebBook that is featured on the Division's internet

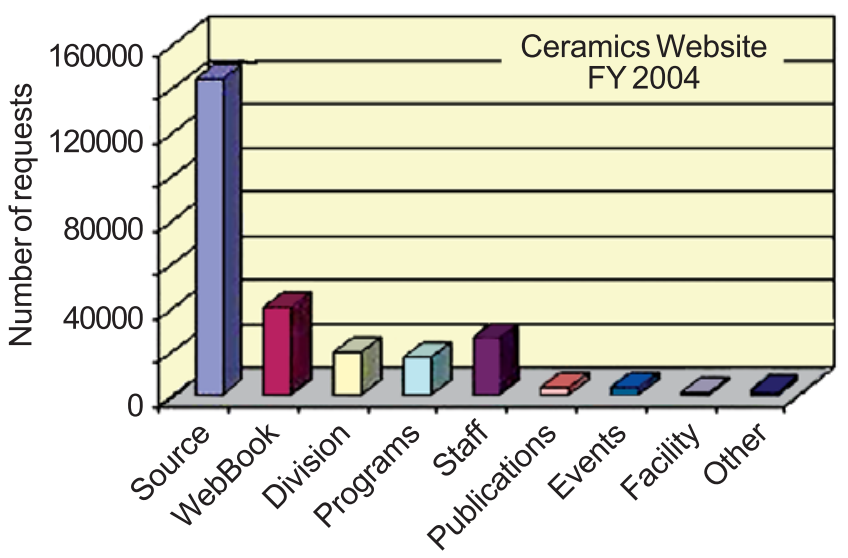

Figure 2: Activity seen by the Ceramics Division's homepage, showing the substantial attraction to property data.

website. This site provides evaluated data for the physical, mechanical, and thermal properties of advanced ceramics, particularly structural ceramics (NIST Standard Reference Database Number 30) and high-temperature superconductors (NIST Standard Reference Database Number 62). The activity on this site, Figure 2, is a clear indicator of both the strong interest and the persistent need for materials data. The site attracts more than 11,000 page requests per month of which approximately $78 \%$ are data downloads (nearly 9000 data tables).

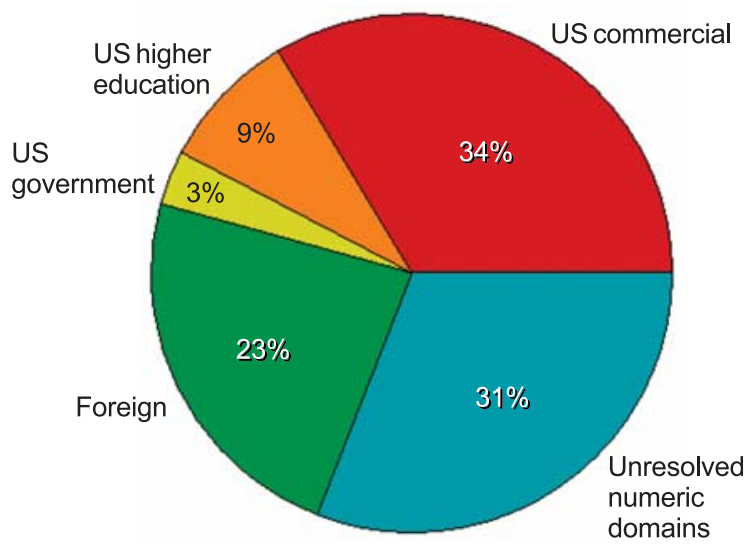

Figure 3: The relative distribution of users downloading data from the Ceramics WebBook.

The distribution of users, Figure 3, shows that all sectors of the ceramics community have a keen interest in the evaluated data provided by NIST.

Another very important mechanism for technology transfer is provided by the Standard Reference Data Act (Public Law 90-3960, 1968). Under the SRD Act, NIST can pursue technology transfer through partnerships that broadly serve the public interest. These partnerships 
typically involve costs in excess of our Congressionally funded base operations and consequently result in small charges for the data products. Two prominent collaborations of this type between the Ceramics Division and private sector nonprofit organizations have brought considerable credit and recognition to NIST.
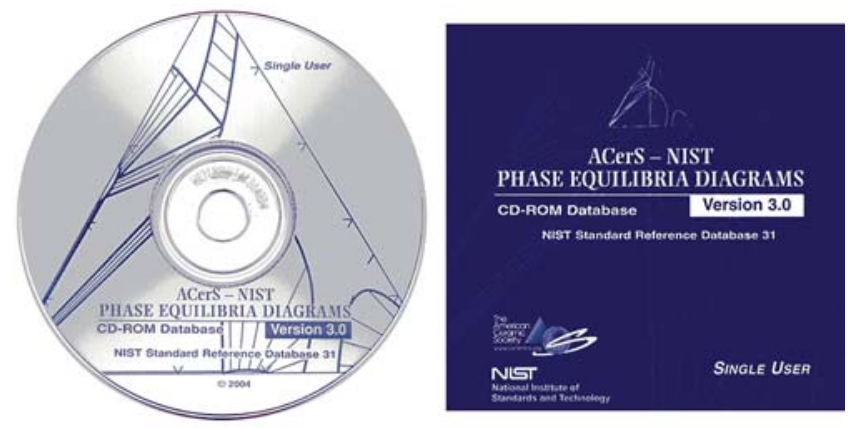

Figure 4: Phase Equilibria Diagrams CD-ROM Database Version 3.0 (NIST Standard Reference Database Number 31), released in March 2004, provides comprehensive coverage of all diagrams and commentaries published to date in the twenty printed volumes of the series.

The longstanding NIST collaboration with the American Ceramic Society (ACerS) on phase diagrams provides a clear example of the remarkable productivity that can result from these partnerships. Now led by Dr. Terrell Vanderah, the ACerS-NIST collaboration achieved one of its most anticipated goals this year, Figure 4, the development of a new computerized version of NIST Standard Reference Database Number 31, Phase Equilibria Diagrams.

Phase equilibria diagrams are used throughout the ceramics industry to understand and control the complex phenomena which increasingly underlie advanced processing and materials performance. These diagrams provide maps of the equilibrium chemical and structural behaviors exhibited by materials and provide critical starting information for the rational design of materials processing schemes, for quality assurance efforts, and for the optimization of the physical and chemical properties of advanced materials.

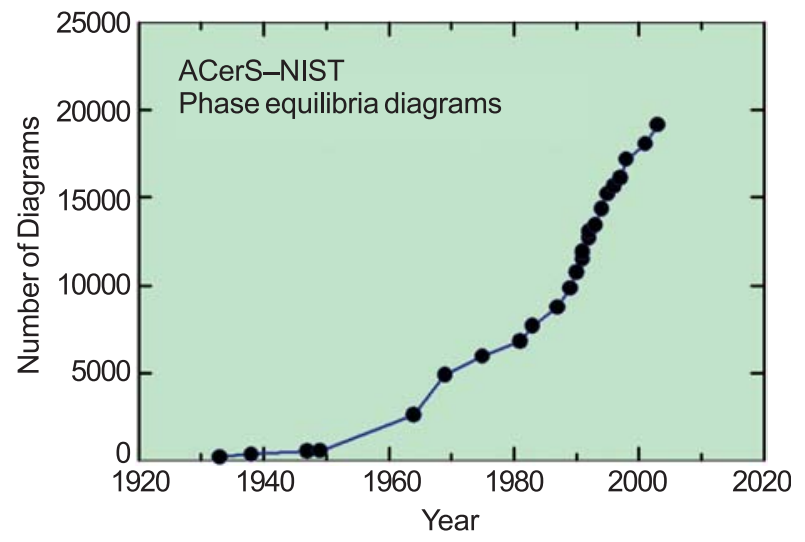

Figure 5: Evolution of the compilation of phase diagrams in the ACerS-NIST collaborative database.
The ACerS-NIST database began with the publication of 178 diagrams in 1933. Since then, the collection has evolved, Figure 5, to contain nearly 20,000 diagrams. Originally, the diagrams were distributed in printed volumes, now amounting to thirteen regular book volumes, four topical volumes, and three annual volumes that serve as supplements to the regular volumes.

Today, both printed and computerized versions are provided and are made available through ACerS.

The newest addition to the series, Figure 6, is the special topical volume, Phase Diagrams for Electronic Ceramics I, edited by Robert S. Roth. This collection concentrates on the technically important oxides of titanium, niobium, and tantalum. Additionally, a newly updated Cumulative Index 2003 was published this year providing comprehensive coverage of all the ACerS-NIST content published to date. All of the new diagrams and commentaries are included in the CD-ROM database.
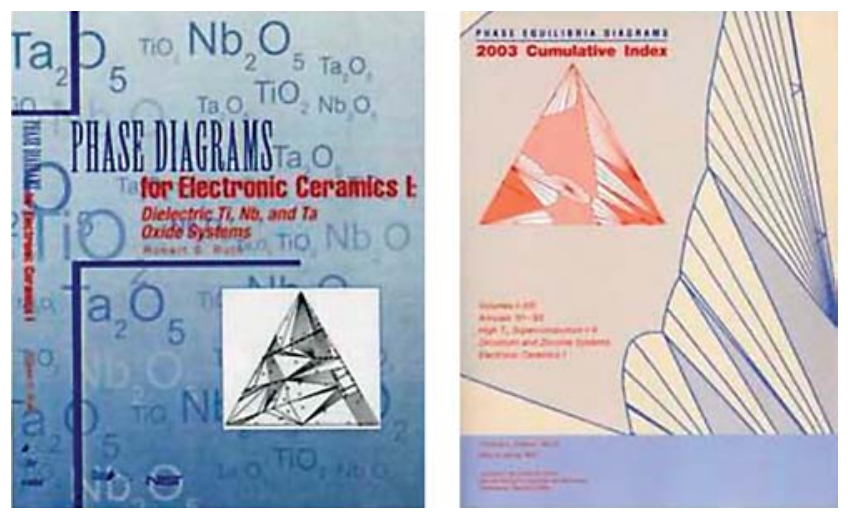

Figure 6: Recent ACerS-NIST printed data products include a topical volume on electronic ceramics and an updated cumulative index for all content published to date.

The mutual commitment of ACerS and NIST to this collaboration was reaffirmed in May of this year with the signing of a new five-year agreement.

The ability to develop partnerships in the public interest also has enabled a successful collaboration, led by Dr. Vicky Karen, between NIST and Fachinformationszentrum Karlsruhe Gesellschaft fur wissenshaftlich-techmische Information $\mathrm{mbH}$ (FIZ). This collaboration, Figure 7, has resulted in the development of NIST Standard Reference Database Number 84, the comprehensive FIZ-NIST Inorganic Crystal Structure Database.

Crystallographic information is critical to efforts to visualize, explain, and predict behaviors of chemicals and materials, as well as to establish the identity of materials in forensic investigations. Literally tens of thousands of structures have been reported. Those reports, however, have varying degrees of reliability and completeness. Consequently, researchers and instrument manufacturers have a 
critical need for evaluated data and the mathematical tools capable of distinguishing good structures from bad structures and compatible data from incompatible data. Through NIST's efforts, the use of standard crystallographic cells, unique reduced cells, and the powerful mathematical matrix methods for the application of group theory have become fundamental to the analysis and identification of crystal structures.

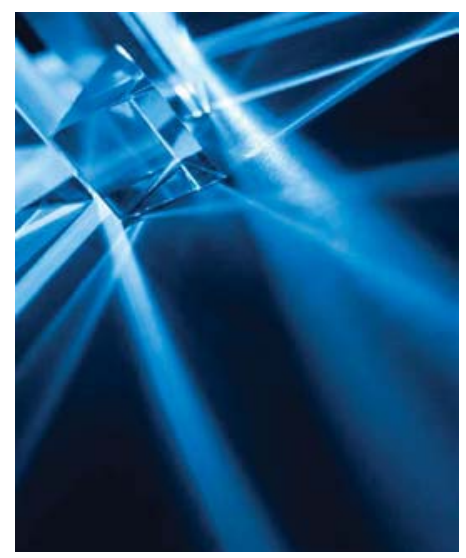

Figure 7: The FIZ-NIST Inorganic Crystal Structure Database contains approximately 70,000 entries providing full structural, three-dimensional crystallographic parameters.

The FIZ-NIST collaboration provides a comprehensive compilation of three-dimensional crystallographic data. The components of a typical entry include data on the conventional cell, the reduced cell, lattice type, space group, lattice parameters, atomic coordinates, d-spacings, chemical name, chemical formula, material class indicators, and literature reference citations.

The collaboration is built on shared and complementary responsibilities. NIST is responsible for the essential scientific and informatic aspects of the work, while FIZ provides data abstracting and related interactions with the International Union of Crystallography, particularly for bibliographic information. Data evaluation is done jointly.

The latest version of the database, released this year, contains more than 70,000 critically evaluated data entries. The database is licensed to research facilities, instrument manufacturers, educational institutions, and individuals. Distributor license agreements with instrument manufacturers are propagated subsequently as user licenses to the instrument buyers. As a result, these data are used every day in defense, aerospace, electronic, petroleum, chemical, and homeland defense related industries, as well as in government and academic research facilities worldwide.

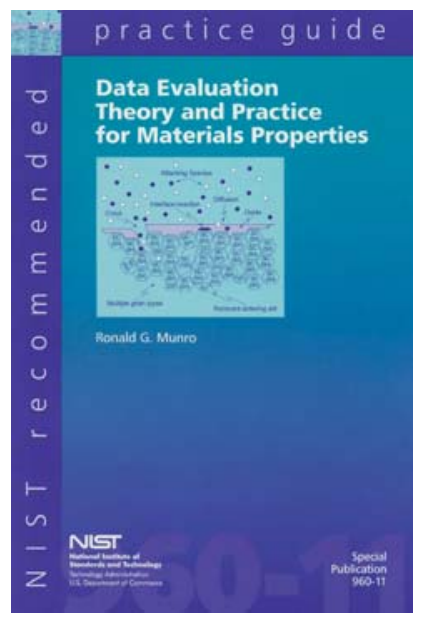

Figure 8: NIST Recommended Practice Guide, SP 960-11, examines pivotal issues in data evaluation.

Additional technology transfer has been achieved through the distribution of NIST Recommended Practice Guide, SP 960-11, Data Evaluation Theory and Practice for Materials Properties, Figure 8.

This Guide presents a unique view of data evaluation as a scientific discipline. A theoretical foundation is developed from the formal underpinnings of materials metrology, and an extensive collection of examples is used to examine the issues of accessibility, reproducibility, consistency, and predictability with distinctions being made among definitive relations, correlations, derived and semiempirical relations, heuristic theories, and value estimates. Critical discussions include the use of properties as parameters in models, the interpretation of ad hoc parameters, and the treatments of procedural properties, response dependent properties, and system dependent data.

Completed in FY2003 and printed in June 2003, more than 1200 hard copies have been issued to interested individuals, and an electronic version in pdf format, available since January 2004, has resulted in the distribution of another 1600 copies.

For a greater appreciation of the full range of data and standards activities in the Ceramics Division, see the tabulation of our products in the section, Databases and Standards, later in this report.

\section{For More Information on this Topic}

P. Schenck, J. Harris, C. Sturrock (Ceramics Division, NIST); A. Belsky (SRD, NIST Technology Services); R. Roth, J. Hastie, M. Harne, A. Mighell (Research Associates, Ceramics Division, NIST); D. Watson (Cambridge Crystallographic DB); M. Mecklenborg, J. You, C. Cedeno, E. Farabaugh, K. Hill, N. Swanson, X.M. Wang (American Ceramic Society); P. Luksch (FIZ); K. Rajan, X. Li, A. Rajagopalan (RPI) 


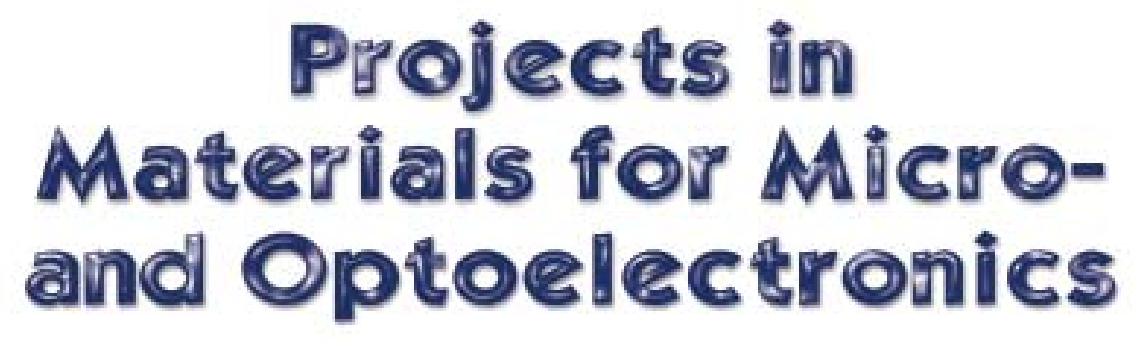




\section{Combinatorial Tools for Materials Science}

Combinatorial materials science has rapidly become a new paradigm for the acceleration of materials research. It is a fast and efficient methodology for materials optimization and discovery, characterized by high throughput, parallel experiments, automated analysis, and massive data sets. NIST's industrial stakeholders are identifying it as the only viable technique for understanding complex materials science systems in a competitive timeframe. Its application to a critical materials science problem in the Si microelectronics industry, the replacement of the gate stack, is expected to drive an inorganic combinatorial materials science program at NIST.

\section{Martin L. Green}

$\mathrm{N}$ IST/MSEL is poised to play several important roles in developing combinatorial materials methodologies, especially as they pertain to industrially important, advanced inorganic materials. Included in this class are materials such as thermoelectrics, dielectric and metal layers for advanced Si CMOS gate stacks, multiferroics, magnetic semiconductors for spintronics, transparent semiconductors, and fuel cell and $\mathrm{H}_{2}$ storage materials. NIST/MSEL has an opportunity to take a lead position in the use of combinatorial methodologies for:

- New, combinatorially-friendly (rapid, local, microscopic measurements) metrologies;

- Materials optimization and development;

- Means of experimentally verifying computed materials properties;

- Tools for determining phase diagrams and other property data; and

- "Data on demand," or "just-in-time-data."

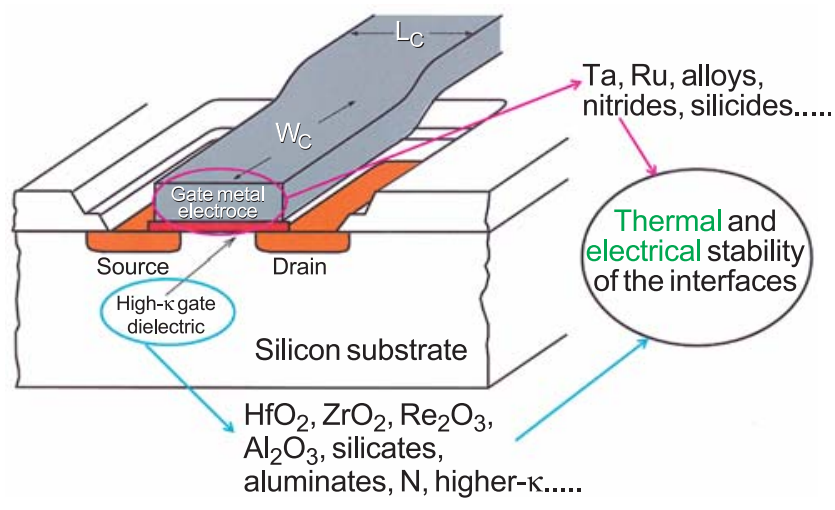

Figure 1: MOSFET device with advanced gate stack.
The advanced gate stack application for Si CMOS is a good example. International Sematech (ISMT) is currently faced with major materials challenges to further miniaturization. Figure 1 schematically illustrates a metal oxide-semiconductor field effect transistor (MOSFET), showing the advanced gate stack which consists of the high- $\kappa(\kappa=$ dielectric constant $)$ gate dielectric and the metal gate electrode. To enable further device scaling, the capacitive equivalent thickness (CET) of the gate stack thickness must be $0.5 \mathrm{~nm}$ to $1.0 \mathrm{~nm}$. This will not be achievable with existing $\mathrm{SiO}_{2}$ /polycrystalline $\mathrm{Si}$ gate stacks. Given the large number of possible choices for these new layers, the only feasible approach to understanding the complex materials interactions that result at the gate dielectric/substrate and gate dielectric/metal gate electrode interfaces is through the application of combinatorial methodologies.

The acquisition of a state-of-the-art combinatorial synthesis tool is expected be one of the cornerstones of the NIST/MSEL effort. In addition, we plan to acquire combinatorially-friendly tools such as a micro-X-ray diffractometer, and to develop new metrologies such as nanocalorimetry for the detection of phase transformations in thin films.

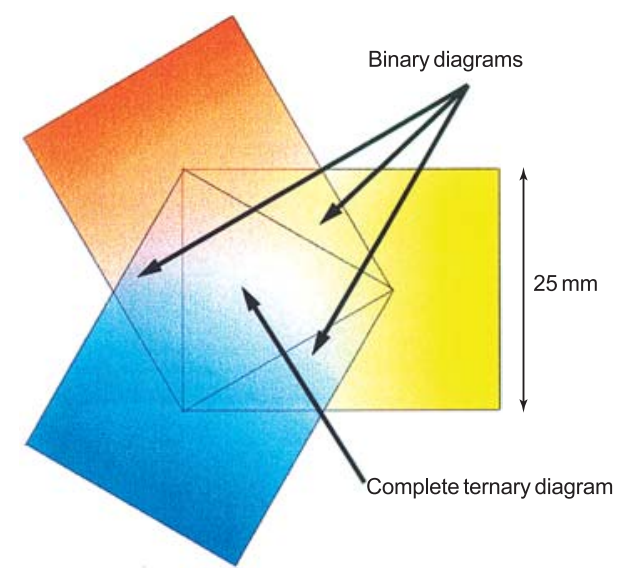

Figure 2: Combinatorial ternary and binary film libraries.

The new combinatorial synthesis tool will be capable of depositing entire ternary and binary libraries in areas as small as $6.5 \mathrm{~cm}^{2}\left(1 \mathrm{in}^{2}\right)$, as is shown in Figure 2.

\section{Contributors and Collaborators}

L. Cook, W. Wong-Ng, P. Schenck (Ceramics Division, NIST); L. Bendersky, A. Davydov (Metallurgy Division, NIST); S. Semancik (CSTL, NIST); J. Suehle, E. Vogel (EEEL, NIST); T. Chikyow (NIMS, Japan); B. Murto (International Sematech); I. Takeuchi (U. of Maryland) 


\section{Spectroscopy, Diffraction, and Imaging of Electronic Materials}

Modern electronic devices have reached the point where fundamental materials properties now limit continued improvement in device performance. Consequently, new materials, their synthesis, their properties, and their interactions with established materials and technologies are in continuous need of development and investigation. The Ceramics Division operates a suite of synchrotron beamlines, at the Advanced Photon Source and at the National Synchrotron Light Source, designed to address critical issues focused on these next-generation materials, including the electronic and structural properties of materials used in advanced electronic applications.

\section{Joseph C. Woicik}

$\mathrm{T}$ he electronics industry is seeking to increase device performance by increasing carrier mobility in silicon by introducing a small tensile strain into the silicon lattice. This is accomplished by the epitaxial growth of a thin silicon layer on a virtual crystalline substrate of relaxed $\mathrm{Si}_{1-\mathrm{x}} \mathrm{Ge}_{\mathrm{x}}$ with $\mathrm{x}$ typically between $10 \%$ and $50 \%$. A critical aspect of this process is the control of defects in the SiGe-alloy layer and the propagation of these defects into the strained-Si film.

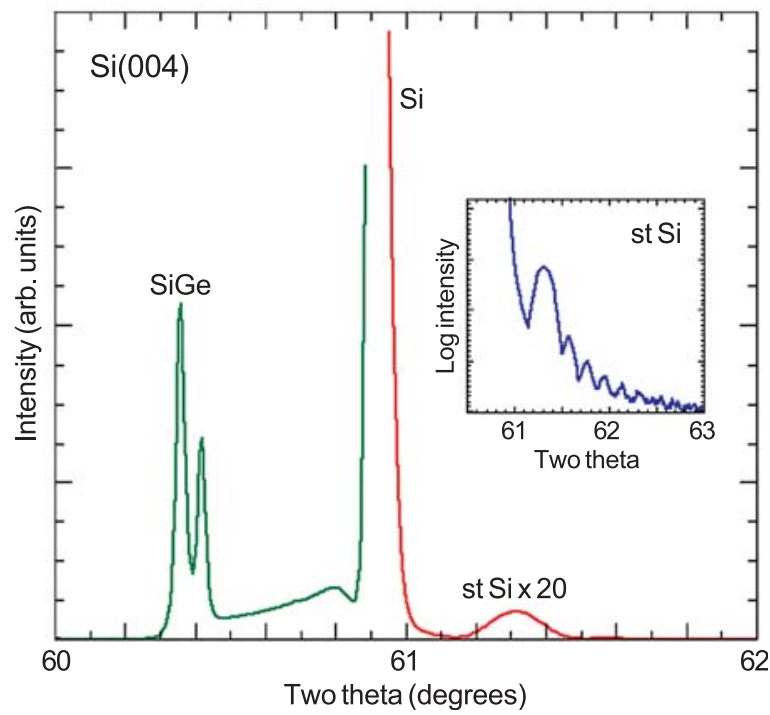

Figure 1: Radial diffraction scan for a strained-Si/Si $i_{x} e_{1-x} / \mathrm{Si}(001)$ semiconductor layered structure.

We have utilized x-ray diffraction and x-ray topography at the UNICAT beamline facility at the Advanced Photon Source to image defects in the SiGe film and to study how these defects propagate into the strained-Si overlayer. Figure 1 shows a high-resolution x-ray diffraction scan around the $\mathrm{Si}(004)$ Bragg condition from a $500 \AA \mathrm{Si}$ film grown on a relaxed $3 \mu \mathrm{m}$ thick SiGe film with a Ge content of $20 \%$. The relaxed $\mathrm{SiGe}$ film was grown on top of a $\mathrm{SiGe}$ layer that was grown on a $\mathrm{Si}(001)$ substrate and graded in composition from $0 \%$ to $20 \% \mathrm{Ge}$ content over a thickness of $2 \mu \mathrm{m}$.

The diffraction from the $\mathrm{SiGe}$ is seen near $2 \theta=60.4^{\circ}$; it appears as a doublet due to a slight composition variation due to the intermediate planarization process. Diffraction from the grade is also apparent. The sharp peak near $2 \theta=60.9^{\circ}$ is diffraction from the Si substrate, and the much weaker peak near $2 \theta=61.3^{\circ}$ is diffraction from the strained-Si film. The Si film has a smaller perpendicular lattice constant than the crystalline Si substrate because it is under in-plane tensile strain due to its epitaxy with the SiGe layer that possesses a larger cubic lattice constant.

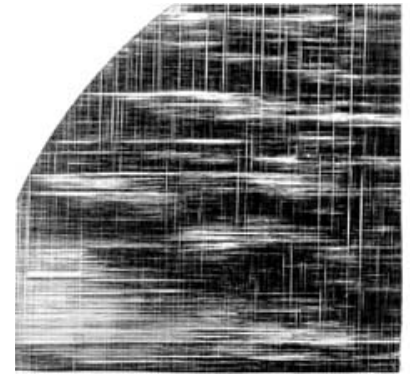

SiGe virtual substrate

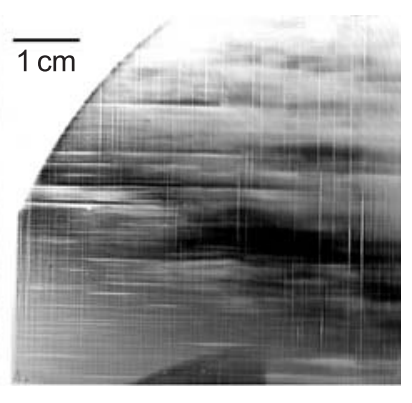

$50 \mathrm{~nm}$ strained silicon film
Figure 2: (113) x-ray topographs of the SiGe film and the strained-Si overlayer.

The differences in lattice constants between the strained-Si film, SiGe layer, and Si substrate make it possible to image the diffraction from each component separately. Figure 2 shows the (113) x-ray topographs recorded with an x-ray energy of $\approx 8.9 \mathrm{keV}$. This diffraction geometry was chosen because it is within Si total external reflection and, therefore, limits the $\mathrm{x}$-ray penetration depth into the sample.

The microstructure of the substrate and film are nearly identical, demonstrating that the misfit-dislocation structure responsible for the relaxation of the $\mathrm{SiGe}$ layer acts as a template for the microstructure of the strained-Si film. The crystallographic nature of these defects and their contribution to the ultimate relaxation of the strained-Si film are currently under investigation.

\section{Contributors and Collaborators}

D. Black, I. Levin (Ceramics Division, NIST); M. Erdtmann, T.A. Langdo (AmberWave) 


\section{Phase Equilibria and Properties of Dielectric Ceramics}

Ceramic compounds with exploitable dielectric properties are widely used in technical applications such as actuators, transducers, capacitors, and resonators or filters for microwave communications. Phase equilibria determination integrated with systematic chemistry-structure-property studies contribute toward the fundamental understanding and rational design of these technologically important materials with improved properties and/or reduced processing costs.

\section{Terrell A. Vanderah, Igor Levin, and Michael W. Lufaso}

Cost and performance are the primary and secondary drivers, respectively, for today's commercial needs in the area of dielectric ceramics. For example, component suppliers and builders of $2 \mathrm{GHz}$ cellular infrastructure are critically impacted by the high cost of tantalum-containing ceramics needed for dielectric resonators: only a single ceramic material, perovskite-like $\mathrm{Ba}_{3} \mathrm{ZnTa}_{2} \mathrm{O}_{9}$ (BZT), is available with the needed properties. Interest is keen to reduce its processing costs or to find an alternative, less-expensive substitute. The results of an experimental phase equilibria study of the system (Figure 1) revealed that BZT ceramics must be processed so that $\mathrm{ZnO}$ volatilization occurs along the two-phase join between $\mathrm{BZT}$ and $\mathrm{Ba}_{8} \mathrm{ZnTa}_{6} \mathrm{O}_{24}$ (8L) to prevent the formation of deleterious air-sensitive compounds high in $\mathrm{BaO}$ content, or dielectrically poor "TTB” type phases.

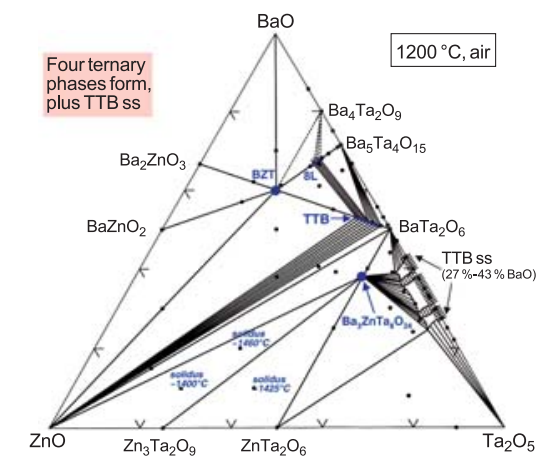

Figure 1: Phase equilibria diagram determined for the system containing the important commercial ceramic $\mathrm{Ba}_{3} \mathrm{ZnTa}_{2} \mathrm{O}_{9}(\mathrm{BZT})$.

A potential replacement for BZT is the analogous compound with less costly niobium, $\mathrm{Ba}_{3} \mathrm{ZnNb}_{2} \mathrm{O}_{9}$. The phase equilibrium diagram determined for this system (Figure 2) provides processing information for this ceramic and clearly indicates that its dielectric loss cannot be improved by liquid-phase sintering in the presence of low-melting, electrically acceptable $\mathrm{ZnNb}_{2} \mathrm{O}_{6}$, because the two compounds do not occur in equilibrium with each other. Such mixtures, instead, will form dielectrically poor impurity phases.

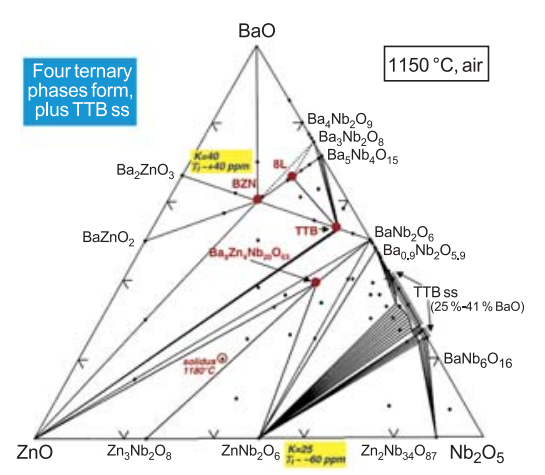

Figure 2: Phase equilibria diagram determined for the system containing $\mathrm{Ba}_{3} \mathrm{ZnNb}_{2} \mathrm{O}_{9}(\mathrm{BZN})$, a possible BZT alternative.

Systematic studies of the $\mathrm{Ba}_{3} \mathrm{MNb}_{2-\mathrm{x}} \mathrm{Sb}_{\mathrm{x}} \mathrm{O}_{9}$ $(\mathrm{M}=\mathrm{Mg}, \mathrm{Ni}, \mathrm{Zn})$, system were carried out to manipulate the crystal chemistry and high-frequency $(>2 \mathrm{GHz})$ dielectric properties by progressive substitution of $\mathrm{Sb}^{5+}$ $\left(d^{10}\right)$ for $\mathrm{Nb}^{5+}\left(d^{0}\right)$. The results showed that control of permittivity and tuning of the temperature coefficient to zero was possible with appropriate substitution. Optimal dielectric losses were obtained for specimens with low $\mathrm{x}$-values and 2:1-type ordered perovskite structures.

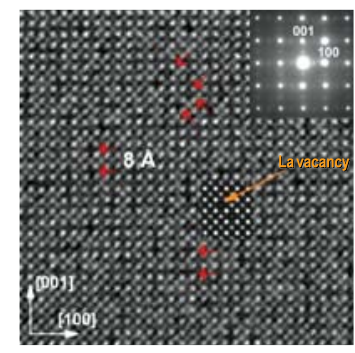

Figure 3: HRTEM image of 50:50 $\mathrm{LaMg}_{1 / 2} \mathrm{Ti}_{1 / 2} \mathrm{O}_{6}-\mathrm{La}_{2 / 3} \mathrm{TiO}_{3}$. Dark crosses denote La vacancies, deduced from image simulations (insert). Short-range order of La vacancies is revealed as pairs of dark crosses in both the [001] and [100] directions and causes diffuse superlattice reflections in the diffraction pattern.

Detailed structural studies of the $\mathrm{LaMg}_{1 / 2} \mathrm{Ti}_{1 / 2} \mathrm{O}_{6^{-}}$ $\mathrm{La}_{2 / 3} \mathrm{TiO}_{3}$ solid solution were carried out to elucidate the origin of the anomalous changes in permittivity and temperature coefficient near 50:50. The results showed that the abrupt changes in properties were accompanied by the disappearance of one octahedral tilting mode and the onset of short-range ordering of La vacancies.

\section{Contributors and Collaborators}

W. Wong-Ng, R.S. Roth, B. Burton, E. Cockayne (Ceramics Division, NIST); J.E. Maslar (Process Measurements Division, NIST); R. Geyer (Radio Frequency Technology Division, NIST); S. Bell (TCI Ceramics) 


\section{Theory and Modeling of Dielectric Materials}

Dielectric ceramics are widely used in applications such as semiconductor gate dielectrics, actuators, capacitors, and resonators or filters for microwave communications. For simple systems, first-principles (FP) methods allow direct calculations of low-temperature dielectric constants. For complex systems, and for rational new materials design, larger scale modeling is required. Multiscale effective Hamiltonian (EH) models derived from FP calculations retain high accuracy and allow phenomena caused by chemical ordering, defects, and changes in temperature to be explored. The EH provides a bridge from FP calculations $(\leq 100$ atoms) to the needed computations with hundreds of thousands of atoms. We are developing a methodology to enable automatic EH generation for real three-dimensional systems.

\section{Eric Cockayne and Benjamin P. Burton}

M aterials that are optimized for industrial applications are generally solid solutions in which the configuration of ions on mixed ion sites strongly affects the physical properties. To model these phenomena, detailed FP calculations are performed on a variety of perfectly ordered systems. For example, $\mathrm{PbMg}_{1 / 3} \mathrm{Nb}_{2 / 3} \mathrm{O}_{3}$ (PMN) is the main component of recently discovered materials with ultrahigh piezoelectric constants. PMN presents a singular challenge, because the details of $\mathrm{Mg}-\mathrm{Nb}$ ordering are not fully known. To better understand PMN, we studied various PMN composition supercells with 15 to 30 atoms. A comparison of fully relaxed structures predicts a ground state ordering different from that previously assumed. The two lowest-energy structures found are most compatible with the "random site model" of PMN deduced from experiment. Calculated infrared spectra (directly related to the dielectric function) also show that the two lowest energy structures are most similar to experiment.

For the $\left(\mathrm{CaAl}_{1 / 2} \mathrm{Nb}_{1 / 2} \mathrm{O}_{3}\right)_{1-\mathrm{x}}-\left(\mathrm{CaTiO}_{3}\right)_{\mathrm{x}}(\mathrm{CAN}-\mathrm{CT})$ system of microwave dielectric interest, a cluster expansion EH model for the low temperature dielectric constant was developed to calculate the dielectric constant for an arbitrary CAN-CT configuration as a function of $\mathrm{x}$ and Ti-Al-Nb ordering. Work is in progress to parameterize similar models for other solid solution systems.

Temperature dependent dielectric properties can be modeled with Monte Carlo and molecular dynamics simulations based on EHs. The EH for a heterovalent solid solution includes the random local electric fields generated by mixing differently charged ions in an alloy. We compiled a catalog of the possible local field directions in the nearest-neighbor approximation. By including local fields in an $\mathrm{EH}$ for $\mathrm{PbSc}_{1 / 2} \mathrm{Nb}_{1 / 2} \mathrm{O}_{3}$ (PSN), we can reproduce the experimental observation that the dielectric peak of PSN, as a function of temperature, is broadened when nanoscale ordered domains are present.

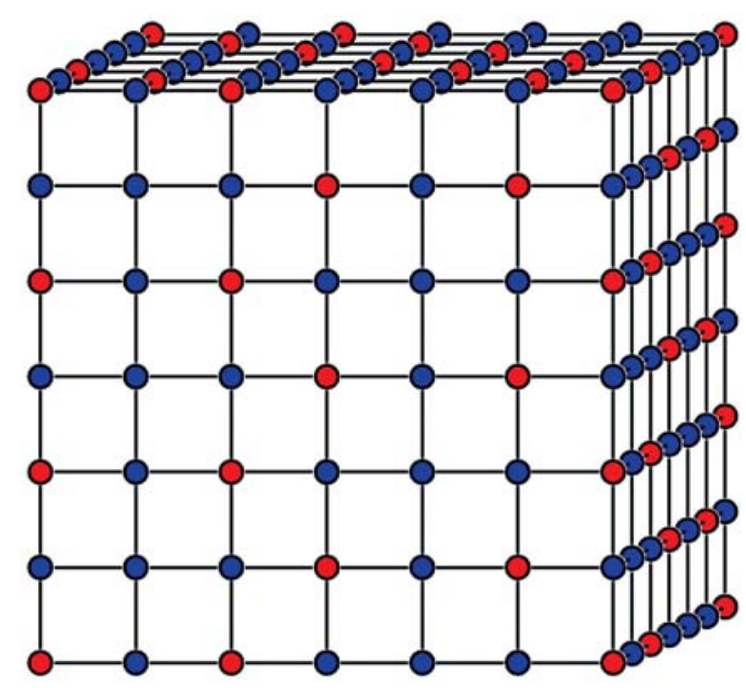

Figure 1: Predicted ground-state ordering of $\mathrm{Mg}$ (red) and $\mathrm{Nb}$ (blue) in $\mathrm{PbMg}_{1 / 3} \mathrm{Nb}_{2 / 3} \mathrm{O}_{3}$.

Defects also affect physical properties. We computed the dipole moment of a $\mathrm{Pb}-\mathrm{O}$ divacancy in $\mathrm{PbTiO}_{3}$ from FP. Its moment is 2.28 e times the distance between the original $\mathrm{Pb}$ and $\mathrm{O}$ sites. The large electric fields produced by such defects have been incorporated into our EH for PSN.

Effective Hamiltonians for dielectrics and ferroelectrics are based on an efficient "lattice Wannier function" (LWF) description of the lattice dynamics of these systems. We have found a principle for automatically generating LWF and have demonstrated that it works on one-dimensional model systems. The method works equally well for chemically ordered systems, disordered systems, and systems with defects. This is a highly promising step towards automatic generation of effective Hamiltonians for real three-dimensional systems, which will make these EH methods accessible to non-experts.

\section{Contributors and Collaborators}

I. Levin, M. Lufaso, S. Tinte (Ceramics Division, NIST); P. Gehrig (NIST Center for Neutron Research, NIST); A. van de Walle (Northwestern U.); S.A. Prosandeev (Rostov State U.); U.V. Waghmare (JNCASR) 


\section{Polymer Photoresists for Next-Generation Nanolithography}

\begin{abstract}
Photolithography, the process used to fabricate integrated circuits, is the key enabler and driver for the microelectronics industry. As lithographic feature sizes decrease to the sub-100 $\mathrm{nm}$ length scale, significant challenges arise because both the image resolution and the thickness of the imaging layer approach the macromolecular dimensions characteristic of the polymers used in the photoresist film. Unique high-spatial resolution measurements are developed to reveal limits on materials and processes that challenge the development of photoresists for next-generation sub-100 $\mathrm{nm}$ lithography.
\end{abstract}

\section{Vivek M. Prabhu}

$\mathrm{P}$ hotolithography is the driving technology used by the microelectronics industry to fabricate integrated circuits with ever decreasing sizes. In addition, this fabrication technology is rapidly being adopted in emerging areas such as optoelectronics and biotechnology requiring the rapid creation of nanoscale structures. In this process, a designed pattern is transferred to the silicon substrate by altering the solubility of areas of a polymer-based photoresist thin film through an acid catalyzed deprotection reaction after exposure to radiation through a mask. To fabricate smaller features, next generation photolithography will be processed with shorter wavelengths of light requiring photoresist films less than $100 \mathrm{~nm}$ thick and dimensional control to within $2 \mathrm{~nm}$.

To advance this key fabrication technology, we work closely with industrial collaborators to develop and apply high-spatial resolution and chemically specific measurements to understand changes in material properties, interfacial behavior, and process kinetics at nanometer scales that can significantly affect the patterning process.

This year, we have continued to apply and advance unique measurement methods to provide structural measurement of fabricated nanoscale structures and new insight and detail into the complex physico-chemical processes used in advanced chemically amplified photoresists. These methods include $x$-ray and neutron reflectivity (XR, NR), small angle $\mathrm{x}$-ray and neutron scattering (SAXS, SANS), near-edge $\mathrm{x}$-ray absorption fine structure spectroscopy (NEXAFS), combinatorial methods, solid state nuclear magnetic resonance (NMR), quartz crystal microbalance (QCM), fluorescence correlation spectroscopy (FCS), and atomic force microscopy (AFM).
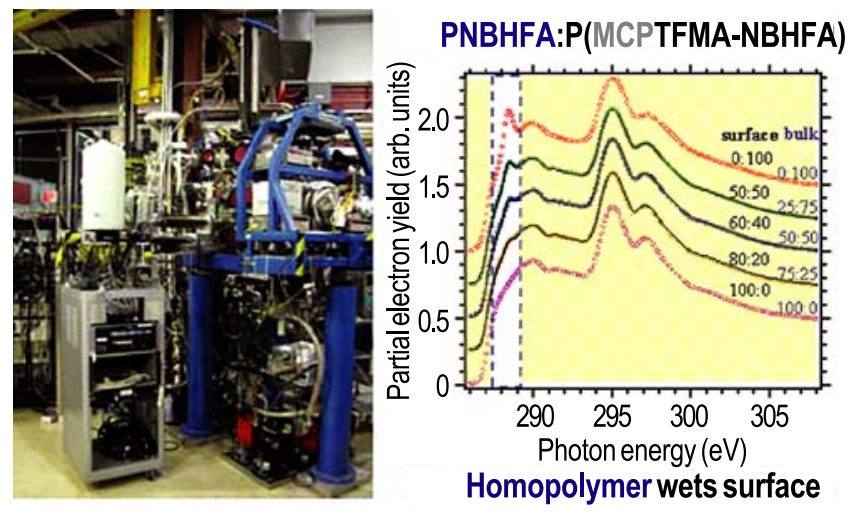

Figure 1: NEXAFS instrument and spectra illustrating a homopolymer enrichment at the film surface for a model $157 \mathrm{~nm}$ photoresist blend. The mismatch in surface versus bulk composition illustrate interfacial limitations for sub $100 \mathrm{~nm}$ structures.

Accomplishments for this past year include: advancement of photoresist-liquid interfaces for immersion lithography and developer distribution in ultrathin films (see highlight); photoresist component segregation (see Figure 1); quantification of the post-exposure bake time on the reaction-diffusion of photoacid 3D deprotection volume; first measurement of immersion and exposure dependence of the surface composition of base additives in $193 \mathrm{~nm}$ resist films; identification of key anti-reflective coating (ARC) components responsible for profile control problems and residual layer formation at the ARC-resist interface; quantification of environmental sensitivity by in-situ processing using NEXAFS; and quantification of the effects of developer and additives on the final resolution of lithographic features using a model reaction-front bilayer geometry.

International SEMATECH has selected NIST in an effort to apply these fundamental measurements to identify materials sources of the fabrication limits of advanced photoresists.

\section{Contributors and Collaborators}

B. Vogt, E. Jablonski, C. Soles, W. Wu, C. Wang, R. Jones, T. Hu, M. Wang, D. VanderHart, C. Chiang, E. Lin (Polymers Division, NIST); D. Fischer, S. Sambasivan (Ceramics Division, NIST); S. Satija (NCNR, NIST); D. Goldfarb, A. Mahorowala, M. Angelopoulos (IBM T.J. Watson Research Center); H. Ito (IBM Almaden Research Center); C. Willson (University of Texas at Austin); R. Puligadda, C. Devadoss (Brewer Science); R. Dammel, F. Houlihan (Clariant Corporation) 


\section{Nanoimprint Lithography}

Nanoimprint lithography (NIL) has emerged as a viable next generation lithography (NGL) capable of transferring physical patterns smaller than $5 \mathrm{~nm}$ into a polymeric film.[1] The production of nanoscale structures enabled by NIL raises new metrology challenges, as the ability to pattern now exceeds the ability to measure and/or evaluate material properties. The objective of this project is to develop high-resolution metrologies that facilitate the development of NIL.

\section{Christopher L. Soles and Ronald L. Jones}

VIL has recently emerged as one of the leading 1 NGL candidates for the semiconductor industry. The 2003 update of the ITRS Semiconductor Roadmap identifies NIL as a strong candidate lithography for the $45 \mathrm{~nm}$ technology node. Silicon Strategies included Molecular Imprints, Inc. (Austin, TX) and Nanonex (Princeton, NJ), two major NIL tool companies, in their "60 Emerging Start-ups" list for 2004. Going beyond CMOS, MIT's Technology Review selected NIL as one of the "10 Emerging Technologies That Will Change the World."[2] The cost efficient and high-resolution nanopattering of NIL will also be beneficial in emerging technologies such as optical communications, data storage, bio devices, nano-electromechanical systems (NEMS), micro-electromechanical systems (MEMS), and sensors, by enabling low-cost nanofabrication.

The NIL concept is elegantly simple. A master pattern with sub- $100 \mathrm{~nm}$ features is fabricated into a hard material ( $\mathrm{Si}$, quartz, Ni, etc.) using high-resolution (but slow) patterning, such as e-beam lithography. The patterns are then transferred into a resist by stamping the hard master mold into a softer polymer or monomer film. The pattern is "set" into the resist film with either heat, to facilitate flow into the mold features, or by using a monomeric (liquid-like) film that cross-links in the mold. These imprints can be repeated multiple times using a single master, greatly reducing the cost of ownership.

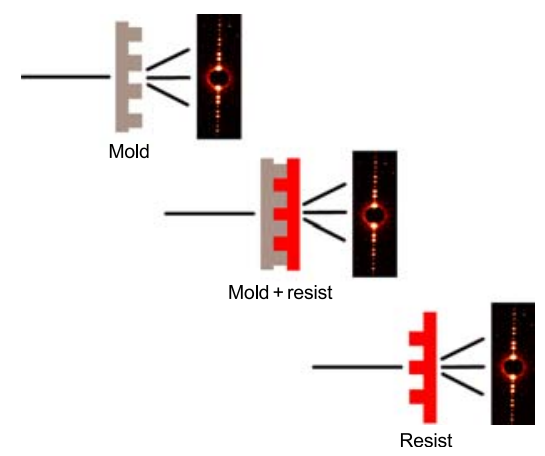

Figure 1: CD-SAXS quantification of the nanoimprint process.
One of the greatest challenges facing NIL is to quantify the fidelity of pattern transfer; traditional shape metrologies struggle when feature sizes approach $5 \mathrm{~nm}$. We are developing critical dimension small angle $X$-ray scattering (CD-SAXS) to quantify 3-D pattern shape, with sub-nm resolution. Since CD-SAXS uses high-energy X-rays that penetrate Si and most mold materials, we can quantify pattern shape in the master mold, on the mold in contact with the resist, and in the final pattern (i.e., fidelity of pattern transfer), as depicted in Figure 1.

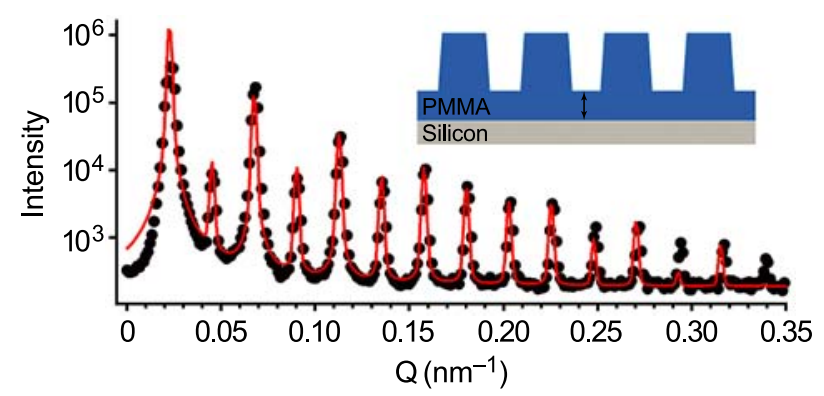

Figure 2: CD-SAXS data (intensity versus scattering vector) for parallel lines and spaces imprinted into a PMMA film.

Figure 2 illustrates CD-SAXS data for parallel lines and spaces imprinted into a poly(methyl methacrylate) (PMMA) film. The data are in black, while the red line is a fit to a trapezoidal cross section. By tilting the sample and fitting several data sets, we determine that the pattern pitch is $(253 \pm 1) \mathrm{nm}$, the average line width is $(146 \pm 1) \mathrm{nm}$, the trapezoid side-wall angle is $(4.2 \pm 0.5)^{\circ}$, and line height is $(180 \pm 1) \mathrm{nm}$.

By providing such accurate structural information, we enable the quantitative evaluation and improvement of imprint processes. Furthermore, we are also developing complementary mechanical and physical property measurements for imprinted polymeric nanostructures. Understanding the unique properties that arise in these nanostructures is crucial to engineering functional and robust polymeric devices at the nanoscale.

\section{References}

1. M.D. Austin, et al., Appl. Phys. Lett. 84, 5299 (2004).

2. Technology Review, Feb, 42-44 (2003).

\section{Contributors and Collaborators}

W. Wu, T. Hu (Polymers Division, NIST); S. Hooker, C. Flannery (Materials Reliability Division, NIST); S. Pang (University of Michigan); C.G. Willson (UT Austin); L. Koecher (Nanonex); D. Resnick, K. Nordquist (Motorola) 


\section{Characterization of Porous Low-k Dielectric Constant Thin Films}

\begin{abstract}
NIST provides the semiconductor industry with unique on-wafer measurements of the physical and structural properties of nanoporous thin films. Several complementary experimental techniques are used to measure the pore and matrix morphology of candidate materials. The data are used by industry to select candidate low-k materials. Measurement methods that may be transferred to industrial laboratories, such as $x$-ray porosimetry, are developed. New methods are being developed to measure patterned low- $k$ samples and to assess the extent of porous structure modification caused by plasma etch.
\end{abstract}

\section{Eric K. Lin and Wen-li Wu}

$\mathrm{T}$ he future generation of integrated circuits requires porous low-k interlayer dielectric materials to address issues with power consumption, signal propagation delays, and crosstalk that decrease device performance. The introduction of nanometer scale pores into a solid film lowers its effective dielectric constant. However, increasing porosity adversely affects other important quantities such as physical strength and barrier properties. These effects pose severe challenges to the integration of porous dielectrics into the device structure.

There is a need for nondestructive, on-wafer characterization of nanoporous thin films. Parameters such as the pore size distribution, wall density, porosity, film uniformity, elemental composition, coefficient of thermal expansion, and film density are needed to evaluate candidate low-k materials. NIST continues to develop low-k characterization methods using a combination of complementary measurement methods including small angle neutron and x-ray scattering (SANS, SAXS), high resolution X-ray reflectivity (HRXR), x-ray porosimetry (XRP), SANS porosimetry, and ion scattering. To facilitate the transfer of measurement expertise, a recommended practice guide for XRP was completed and will be available for industrial customers.

In collaboration with industrial and university partners, we have applied existing methods to new low-k materials and developed new methods to address upcoming integration challenges. A materials database developed in collaboration with International SEMATECH is used extensively by SEMATECH and its member companies to help select candidate materials and to optimize integration processing conditions. This year, we also addressed the effects of the ashing/plasma etch process on the low-k material during pattern transfer. Often, surfaces exposed to ashing/plasma densify and lose terminal groups (hydrogen or organic moiety) resulting in increased moisture adsorption and, thus, increased dielectric constant. HRXR measurements enable quantification of the surface densification or pore collapse in ashing-treated and/or plasma-treated blanket films.
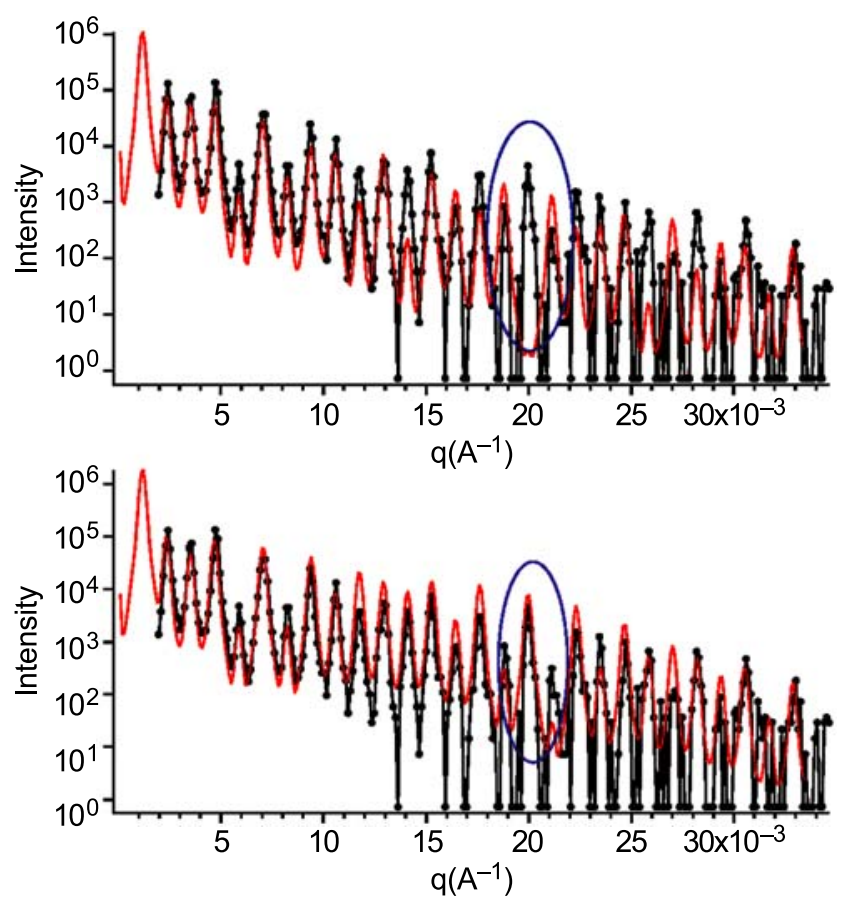

Figure 1: SAXS data of a test line grating created in a porous low-k film. Experimental data - solid symbol; model fit - red line. The top data was fit with a uniform cross-section whereas the bottom data was fit with a dense skin layer. Regions outlined by blue circle highlight the difference between these two models.

A new method using SAXS was also developed to investigate the effect of plasma etch on patterned low-k films. Any densification of the sidewall may be observable by $x$-ray scattering from the cross-section of a patterned nanostructure. Preliminary SAXS work was carried out at Argonne National Laboratory using line gratings of low-k material. The feasibility of this approach is shown in Figure 1 where a surface layer densification is needed to fit the SAXS data.

\section{Contributors and Collaborators}

H. Lee, C. Soles, R. Hedden, M. Silverstein, T. Hu, R. Jones, D. Liu, B. Vogt, B. Bauer (Polymers Division, NIST); C. Glinka (NIST Center for Neutron Research); Y. Liu (International SEMATECH); Q. Lin, A. Grill, H. Kim (IBM); M. Ko (LG Chem.); H. Fu (Novellus); J. Quintana, D. Casa (Argonne National Laboratory); K. Char, D. Yoon (Seoul National University); J. Watkins (University of Massachusetts - Amherst) 


\section{Physical Properties of Thin Films and Nanostructures: Nanoporous Low- $\kappa$ Dielectric Films}

\begin{abstract}
Nanoporous low-dielectric-constant (low-א) films present considerable implementation challenges for the microelectronics industry and also raise new scientific questions. We apply optical methods to these films to evaluate such critical properties as density/porosity, Young's modulus, and Poisson's ratio. Our techniques allow measurement of properties that are otherwise difficult to determine and shed light on how the mechanical properties depend on porosity.
\end{abstract}

\section{Colm Flannery and Donna C. Hurley}

$\mathrm{C}$ urrent miniaturization trends in microelectronics require faster device switching. This need can be at least partially met by lowering the resistance-capacitance factor of the dielectric materials. One promising solution is to introduce nanometer-sized pores that reduce the dielectric constant $\kappa$. Unfortunately, introduction of porosity may lead to a drastic reduction in stiffness (Young's modulus), adversely affecting the material's chances of surviving the fabrication process. In addition to predicting and ensuring process reliability, accurate values of a film's mechanical properties are needed to model the mechanical behavior of the resulting microelectronics device. New tools are needed to characterize these relevant thin-film properties (e.g., Young's modulus and Poisson's ratio v), as well as to better define their dependence on porosity.

In FY04, we evaluated Poisson's ratio $v$ in methylsilsesquioxane (MSSQ) polymer films of varying porosity with Brillouin light scattering. In this technique, a Fabry-Perot interferometer detects frequency-shifted photons scattered by ambient thermal phonons in the material. The frequency shift of the photons is characteristic of the acoustic phonon modes in the film. Values of $v$ were determined from measurements of both longitudinal and surface acoustic wave modes. Figure 1 reveals that $v$ decreases as porosity increases. The results

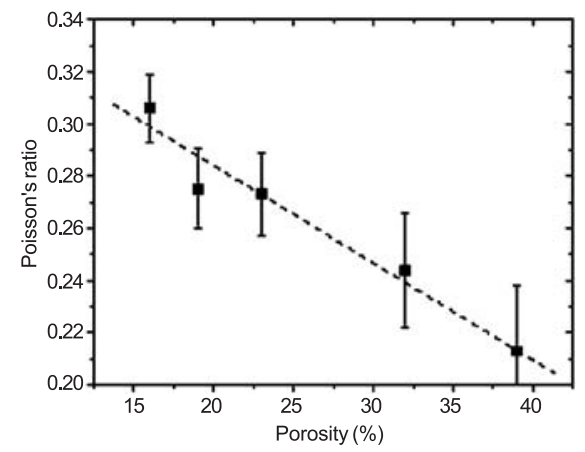

Figure 1: Measured Poisson's ratio vs. porosity for MSSQ films.

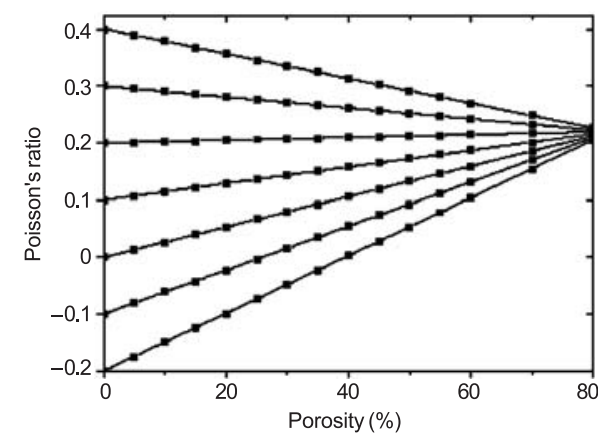

Figure 2: Predicted dependence of Poisson's ratio on porosity.

of an existing finite-element model, shown in Figure 2, predict that $v$ will either decrease or increase depending on the initial $v$ of the matrix material. In addition, $v$ tends towards a constant value of 0.2 for all materials at high porosity. Our measurements are consistent with the model. However, they show a much larger rate of decrease in $v$, probably because a percolation threshold had been reached. Quantitative results like these will prove valuable for modeling of structures involving low- $\kappa$ films. The results also yield insight into the porosity dependence of $v$, about which very little is known.

A second method to determine thin-film properties involves the frequency-dependent dispersion of lasergenerated surface acoustic waves (SAWs). In FY04, efforts concentrated mainly on the development of a standard SAW data analysis procedure. With careful signal processing that minimizes sensitivity to noise and maximizes the frequency range of the measured signals, we can extend the frequency range of our measurements by $20 \%$. The result is a significant reduction in the uncertainty of the extracted film properties. The method lends itself to automatation and has allowed us to inspect films less than $300 \mathrm{~nm}$ thick. (Previously, films less than $500 \mathrm{~nm}$ thick were challenging.)

The improved measurement procedure, combined with new multilayer Green's function analysis software, has allowed us to inspect multilayer structures.

With these capabilities, we have extracted properties of thin $(50-100 \mathrm{~nm})$ capping layers of stiffer materials on top of more compliant functional films. In addition, the Young's modulus, density, and thickness of the underlying dielectric films were evaluated.

\section{Contributors and Collaborators}

S. Kim, V. Tewary (Materials Reliability Division, NIST); Y. Liu (International Sematech); J. Wetzel (Tokyo Electron America) 


\section{Micrometer-Scale Reliability: Chip-Level Interconnects}

Semiconductor manufacturers require increasingly demanding thermal and electrical performance from chip-level interconnects, as feature sizes continue to shrink. Reliability of such small structures then becomes compromised by failure modes unseen by earlier generation interconnect systems.

Our studies address thermal and electrical behaviors of interconnects under conditions of extreme stressing. In particular, we concentrate on the roles of localized stress and variations in micro nanostructure in limiting interconnect performance and lifetime. We performed basic studies on aluminum-based structures undergoing $A C$ stressing at high current density $\left(>10 \mathrm{MA} / \mathrm{cm}^{2}\right)$ as well as measurements of electrical behavior in advanced sub-100 $\mathrm{nm}$ copper and silver wires.

\section{Robert R. Keller, Roy H. Geiss, and Yi-Wen Cheng}

$A^{C}$ stressing at high-current density and low frequencies is used to simulate the thermomechanical fatigue that can occur during a microelectronic device's operational lifetime, due for example to power cycling, energy saving modes, and to application-specific thermal fluctuations. Cyclic Joule heating takes place, leading to differential thermal expansion between metallic interconnects and surrounding substrates or passivation layers. The resulting damage is due to fatigue mechanisms known to operate in bulk metals, but with the constraints associated with thin films imposed. This year, we performed detailed transmission electron microscope (TEM) studies of the deformation mechanisms, supplementing last year's electron backscatter diffraction studies.

Figure 1 shows a pair of TEM images, bright field (above) and weak beam dark field (below), taken from the same region of a cross section of a severely stressed aluminum interconnect. The image shows a high density of dislocation loops, as indicated by arrows. Analysis of diffraction contrast suggests these to be prismatic loops, where the Burgers vector lies normal to the plane of the loop. Such dislocations can be formed by the coalescence of lattice vacancies into a platelike arrangement. Probable sources of vacancies in thermomechanically deformed metal films include grown-in vacancies or those formed during cross-cutting of screw dislocations.

The existence of prismatic loops suggests a possible means for estimating the local temperature rise during a heating cycle. Such loops are known to disappear after prolonged exposure to high temperatures, as vacancies

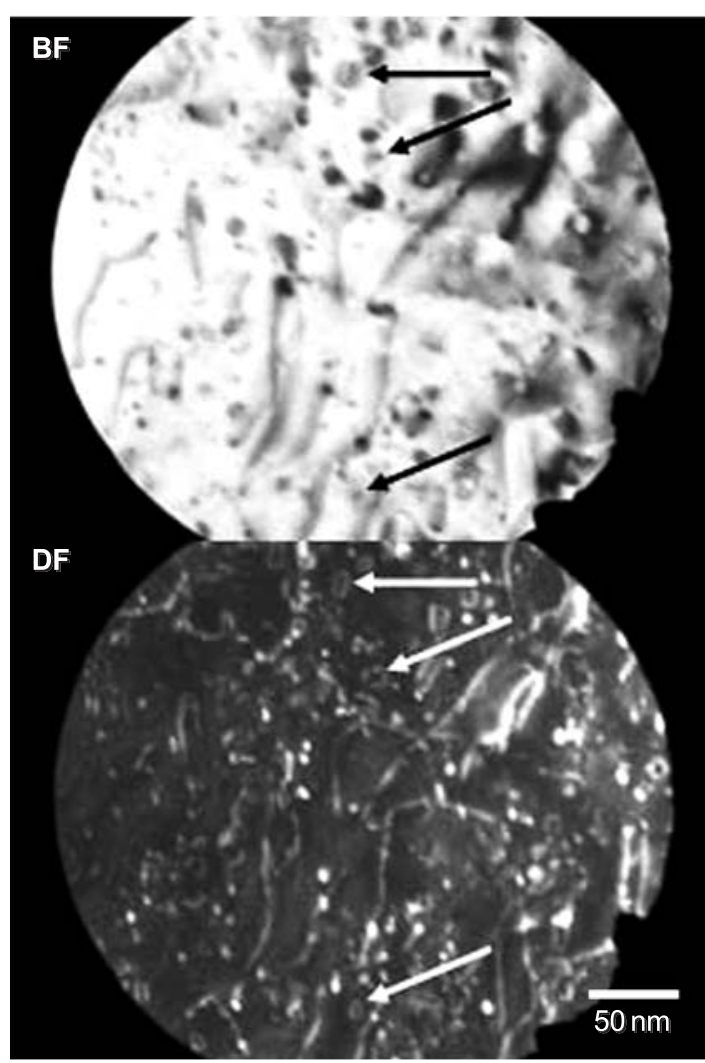

Figure 1: TEM images showing dislocations induced during high-current density AC stressing in aluminum.

diffuse out of the film. The observation of these loops after two hours of testing suggests that there was little sustained exposure above approximately $150{ }^{\circ} \mathrm{C}$, which is consistent with our estimates based on electrical resistance measurements. Both a conference proceedings and an archival manuscript are in progress.

We also completed, in collaboration with Metallurgy Division, a study of linewidth effects on resistivity in narrow $(<50 \mathrm{~nm})$ silver interconnects fabricated by patterned electrodeposition. We found that resistivity increases could be attributed to electron scattering from interconnect surfaces. More details are provided in the Metallurgy Division Annual Report for fiscal year 2004. A manuscript is now in press for Journal of Applied Physics.

\section{Contributors and Collaborators}

D. Josell (Metallurgy Division, NIST); C. Burkhard (Clarkson University); C. Witt (Cookson ElectronicsEnthone) 


\section{Electrical Properties of On-Chip Interconnections}

Copper wiring in on-chip electrical conductors has reached dimensions so small that electrical resistivity is no longer constant. The higher resistivity induces greater power dissipation, aggravating chip cooling problems. We have quantified the microstructural factors behind this increase for silver wires, where silver has the highest electrical conductivity of any metal.

\section{Daniel Josell and Thomas P. Moffat}

$\mathrm{A}^{\mathrm{s}}$ dimensions of transistors in integrated circuits shrink, the dimensions of the metal wires connecting them shrink as well. Dimensions of the wires, and internal grains, are now at approximately $100 \mathrm{~nm}$, similar to the intrinsic mean-free-path lengths of the conduction electrons. Scattering from wire surfaces and grain boundaries is significantly reducing electrical conductivity in such small conductors.

Upcoming dimensional requirements, as specified in the International Technology Roadmap for Semiconductors, require determination of the origin and magnitude of the resistivity increase in order to direct research that might mitigate these size effects.

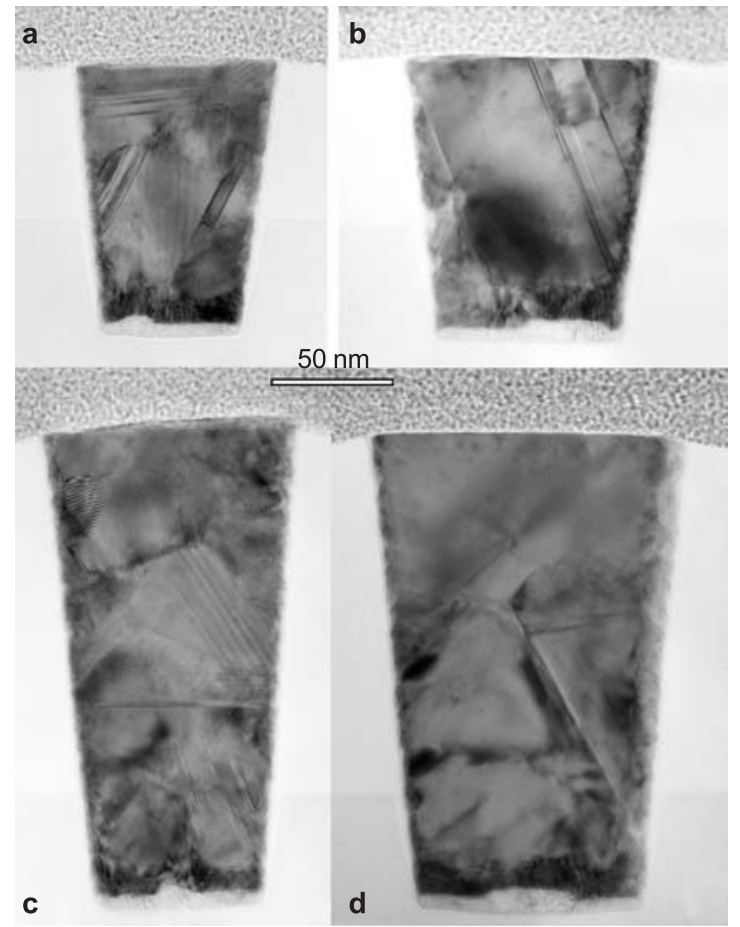

Figure 1: Transmission electron microscope images of silver wires cross-sectioned after electrical measurement. The higher aspect ratio (height/width) wires contain multiple grains while the lower aspect ratio wires contain only twin boundaries.
To address these issues, we have measured size-dependent resistivity of silver wires $100 \mathrm{~nm}$ to $300 \mathrm{~nm}$ tall for widths ranging from approximately $50 \mathrm{~nm}$ to $840 \mathrm{~nm}$, produced by electrodeposition on patterned wafers followed by a combined chemical mechanical planarization and oblique ion-polishing process. Silver was selected because of its high electrical conductivity and our recent development of a bottom-up "superfill”" process for creating void-free and seam-free wires (Figure 1).

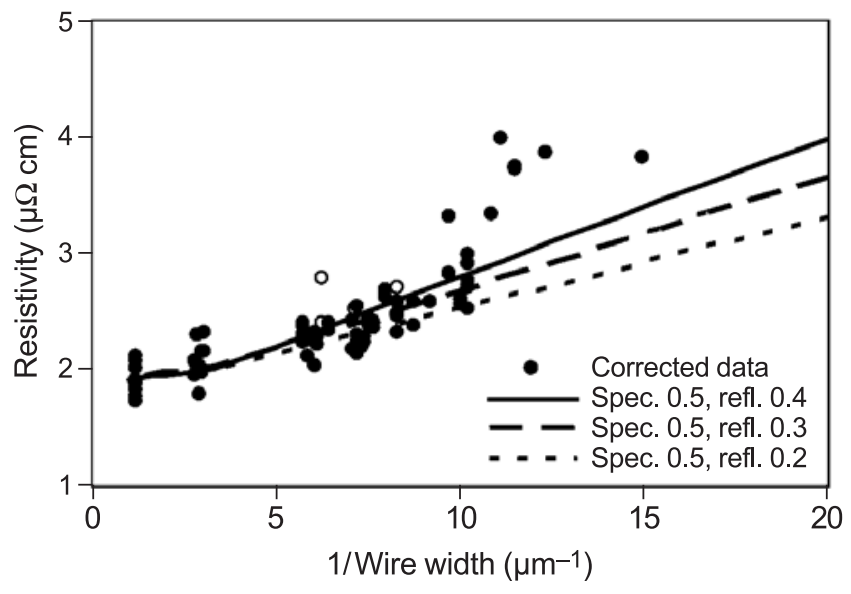

Figure 2: Electrical properties of $300 \mathrm{~nm}$ tall silver wires as a function of the wire width. Curves are the result of calculations including intrinsic, surface and grain boundary scattering of electrons. The sharp increase at $\approx 100 \mathrm{~nm}$ is associated with defects.

To interpret the resistivity data, the Fuchs-Sondheimer formalism (diffuse scattering of electrons on the wire surfaces) was extended to permit a nonzero specular component for surface scattering. The analysis used an existing formalism to account for grain boundary scattering. The resulting equations permit quantitative comparison to experiment (Figure 2).

Significantly, the analysis shows that surface scattering alone is not capable of explaining the observed behavior. Grain boundary scattering is found to contribute a similar, or larger, amount to the overall increase. As a result, the potential payoff of increasing the grain size to decrease grain boundary scattering is recognized to be at least as large as that which might be obtained by smoothing surfaces to increase specularity.

\section{Contributors and Collaborators}

G. McFadden (Mathematical and Computational Sciences Division, NIST); R.R. Keller, Y.-W. Cheng (Materials Reliability Division, NIST); C. Witt (CooksonEnthone); C. Burkhard, Y. Li (Clarkson University) 


\section{Electronic Packaging and Components: Packaging Reliability}

\begin{abstract}
We are developing methods to examine materials and interfaces in electronic packaging applications and elucidate the damage mechanisms. Our current focus is on advanced packaging structure, embedded passive materials, and thin metal films by use of thermal microscopy to measure heat flow and thermal properties on increasingly smaller size scales.
\end{abstract}

\section{Andrew J. Slifka}

\section{Technical Description}

$\mathrm{T}_{\mathrm{h}}^{\mathrm{h}}$ he microelectronics industry is moving toward components of higher density and smaller size that use less expensive materials. One result of this is that reliability becomes an increasing concern due to coefficient of thermal expansion (CTE) mismatch and increased heat flow. As size decreases and functionality increases, thermomechanical fatigue becomes a factor in more areas of the microelectronics industry, including lead-free solders and interconnects.

We are investigating the structural damage induced from CTE mismatches between the various component materials in electronic packages to determine initiation of damage and the ultimate failure mechanisms. Thermal microscopy is used to measure changes in interfacial thermal resistance in order to detect the onset and continuation of thermomechanical damage, leading to electrical failure at interfaces.

We are developing new measurement methods using scanned-probe microscopy (SPM) in order to characterize packages and measure thermal conductivity of thin films at increasingly smaller size scales.

\section{Accomplishments}

We have completed measurements on industrial embedded resistors (see Figure 1), making comparative measurements between the SPM thermal system and the IR microscope.

We have made measurements of films by use of thermal SPM in a mode that uses the probe tip simultaneously as a point-source heater and a resistive element in a Wheatstone bridge circuit. For example, we have made preliminary measurements using various thicknesses of gold films on different substrates, and have compared a new theory, developed by our collaborators, to our measurements. Figure 2 shows data from a suite of these measurements. We will add interfacial modifications to the samples and to the theory in order to

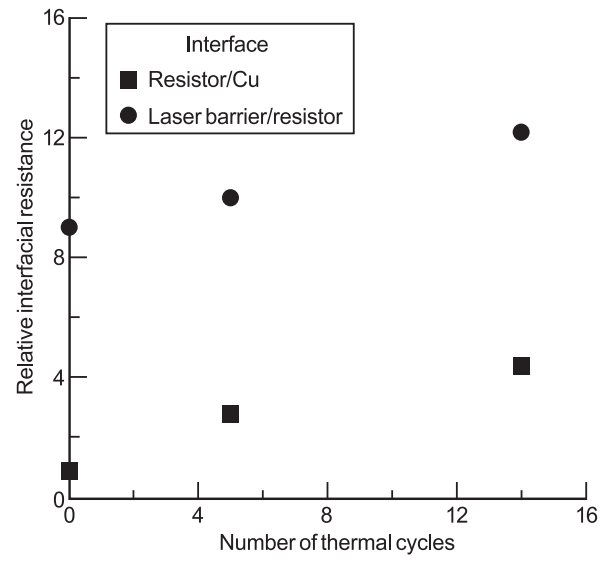

Figure 1: Results of thermal SPM measurements on embedded resistor materials.

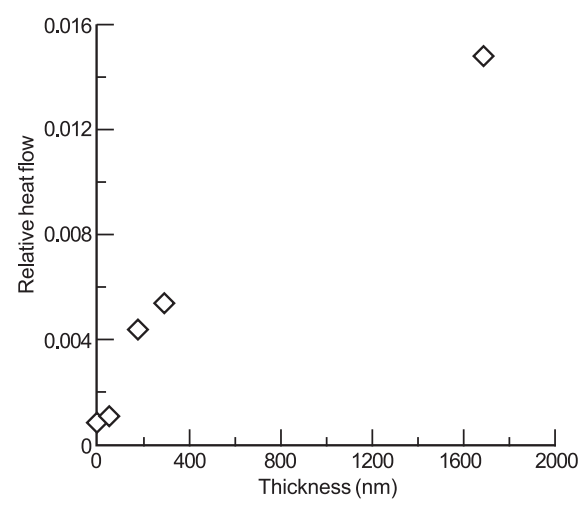

Figure 2: Thermal SPM measurement results on various thicknesses of gold films on glass substrates.

make the measurement method applicable to films used in industry. In addition, we have been using this measurement method on diamond-like-carbon (DLC) films. These industrial films are made from a polymer precursor, which allows easy and inexpensive coating onto various substrates. The thermal conductivity of these films, and how they compare to conventionally processed DLC films, is being investigated.

Finally, we are also measuring thermal conductivity of lead-free solder balls and interfacial thermal resistance between alloys and intermetallics.

\section{Contributors and Collaborators}

J. Felten (DuPont Technologies, Research Triangle Park, NC); R. Snogren (SAS Circuits, Inc., Littleton, $\mathrm{CO}$ ); K. Cole (University of Nebraska, Lincoln, NE); S. Joray (Cenymer Corp., Longmont, CO); F. Hua (Intel Corp., Santa Clara, CA) 


\section{Electronic Packaging and Components: Acoustic Characterization}

\begin{abstract}
Bulk acoustic techniques have been developed and applied to the characterization of a variety of materials, with particular emphasis on piezoelectric crystals for electronic oscillator and filter applications. The research includes metrology of mode-selective noncontacting resonant spectroscopy, characterization of physical mechanisms that degrade performance of piezoelectric compounds with the structure of langasite, and determination of elastic constants of body-centered-cubic Ti and the shape-memory alloy NiTi.
\end{abstract}

\section{Ward Johnson, Sudook Kim, and Carlos Martino}

$\mathrm{O}$ ver the past several decades, the Materials Reliability Division has established special capabilities in a variety of resonant acoustic techniques, including resonant ultrasound spectroscopy (RUS), timedomain resonance methods, noncontacting transduction, Marx-oscillator measurements, and torsional-pendulum measurements. The combined capability of these resonance systems enables elastic constants and anelastic damping to be measured from $4 \mathrm{~K}$ to $1100 \mathrm{~K}$. The division also has implemented several conventional pulse-echo systems, including immersion scanning. During FY04, research was focused primarily on noncontacting mode selective metrology, analysis of anelastic damping in piezoelectrics, measurements of the temperature dependence of elastic constants of a shape-memory alloy, and determination of elastic constants of Ti in its high-temperature body-centered-cubic phase.

Langasite $\left(\mathrm{La}_{3} \mathrm{Ga}_{5} \mathrm{SiO}_{14}\right)$ and several of its isomorphs, including langatate $\left(\mathrm{La}_{3} \mathrm{Ga}_{5.5} \mathrm{Ta}_{0.5} \mathrm{O}_{14}\right)$, have attracted significant attention in recent years as piezoelectric materials for improved electronic oscillators and filters. Research in our lab over the past year has sought to identify the dominant anelastic mechanisms that degrade the performance of these materials. An algorithm has been developed for fitting the measured temperature and frequency dependences of the mechanical $Q$ to a superposition of loss mechanisms. For langasite, three point-defect relaxations are shown to be superimposed on a contribution that increased monotonically with temperature. In langatate, very similar anelastic effects appear, with distributions of activation energies that apparently arise from variations in residual stress.

To enable robust measurement of elastic constants of trigonal piezoelectric crystals, such as langasite,

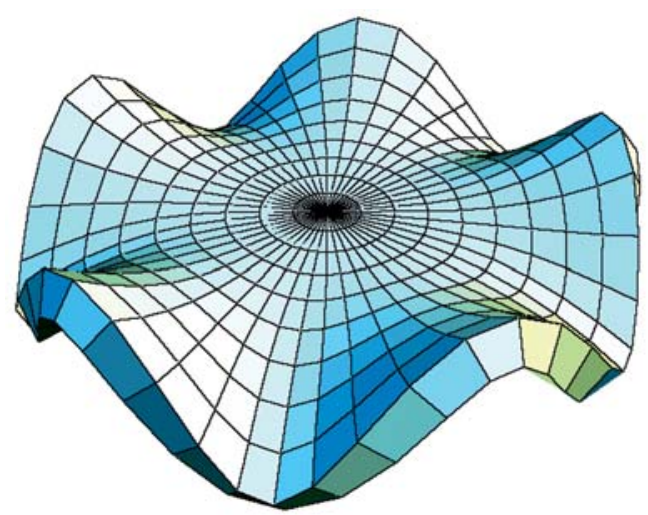

Figure 1: Vibrational mode of a sapphire disk.

methods of selectively measuring and analyzing resonant frequencies of modes with specified symmetry have been developed. This approach overcomes errors in analysis that can arise from misidentification of modes. The research has included measurements and analysis of quartz cylinders and sapphire disks. Noncontacting piezoelectric coupling with switchable electrode configurations and Ritz inversion algorithms including full symmetry information have been developed.

In collaboration with Los Alamos National Lab, analysis of resonance measurements on polycrystalline $\mathrm{Ti}$ at $1270 \mathrm{~K}$ were performed. Polycrystalline elastic constants were determined using the Ritz method. Then, the Kröner model was used to estimate monocrystalline constants from these polycrystalline constants.

Measurements of the temperature dependence of the elastic constants of the shape-memory alloy NiTi have been pursued in collaboration with the Mechanical Engineering Department of the University of Colorado. This study determined the effects of metallurgical treatments on the martensitic transition temperature and elastic constants. Pulse-echo measurements of elastic constants were performed on cold-drawn and hot-rolled specimens from $155 \mathrm{~K}$ to $300 \mathrm{~K}$. Large Poisson ratios $(>0.4)$ reflect the material's superelasticity. Information was obtained on the hysteresis in elastic constants arising from the phase transition, which is a subject of controversy.

\section{Contributors and Collaborators}

P. Heyliger (Colorado State Univ.); M. Dunn, K. Gall (Univ. of Colorado, Mechanical Engineering Dept.); H. Ogi (Osaka Univ., Japan); H. Ledbetter (Los Alamos National Lab); A.Teklu (Univ. of Mississippi, Oxford) 


\section{Lead-Free Surface Finishes: Sn Whisker Growth}

As the microelectronics industry moves towards Pb-free assemblies, reliability has become a major concern. Pb-free coatings of nearly pure tin, used as a protective layer to maintain solderability on $\mathrm{Cu}$ leadframes and connectors, tend to grow "whiskers" which can cause shorts across component leads. Copper additions to the Sn protective layer have been considered by industry since the $\mathrm{Sn}-\mathrm{Cu}-\mathrm{Ag}$ is likely to be the Pb-free bulk solder of choice for industrial applications. However, measurements made at NIST indicate that the addition of $\mathrm{Cu}$ tends to promote whisker growth. Our goal is to better understand how copper and other solutes impact the growth of whiskers on electrodeposited Sn.

\section{Gery R. Stafford, William J. Boettinger, and Kil-Won Moon}

W hiskers are generally believed to grow to relieve residual stress in tin and other coatings. However, the origin of this stress has not been definitively determined. The addition of certain solutes, such as $\mathrm{Bi}$ and $\mathrm{Pb}$, are known to retard whisker growth in electrodeposited tin coatings, while solutes, such as $\mathrm{Zn}$ are known to promote growth. Previous work at NIST has shown that low levels of $\mathrm{Pb}$ reduce the residual compressive stress in $\mathrm{Sn}-\mathrm{Pb}$ electrodeposits, while the addition of $\mathrm{Cu}$ increases the residual compressive stress. Current research focuses on the influence of solute type and concentration on residual film stress, stress relaxation, and whisker formation.

Metallographic analysis of $\mathrm{Sn}-\mathrm{Cu}$ electrodeposits indicates that the $\mathrm{Cu}$ is present as the $\mathrm{Cu}_{6} \mathrm{Sn}_{5}$ intermetallic compound (IMC) which is observed both in the grains and along the grain boundaries. It is unlikely that significant amounts of this complex IMC are formed directly during deposition. Rather, we believe that the as-deposited alloy is a supersaturated solid solution of $\mathrm{Cu}$ in $\mathrm{Sn}$ that transforms to an equilibrium mixture of $\mathrm{Sn}$ and $\mathrm{Cu}_{6} \mathrm{Sn}_{5}$ either during or soon after deposition.

In solid solution, $\mathrm{Cu}$ is presumed to occupy interstitial sites within relatively large square channels that exist in the body-centered tetragonal (bct) Sn structure. This gives rise to the anomalously fast diffusion of $\mathrm{Cu}$ in the c-direction of the $\mathrm{Sn}$ lattice. In this configuration, it is assumed that the $\mathrm{Cu}$ does not alter the lattice volume of the $\mathrm{Sn}$. It is further presumed that as $\mathrm{Cu}_{6} \mathrm{Sn}_{5}$ precipitates within the deposit, the $\mathrm{Cu}$ exits the interstitial sites in the Sn phase and occupies volume more typical of its atomic size; thus, the volume of the entire deposit increases.
We estimate the volumetric strain for $\mathrm{Cu}_{6} \mathrm{Sn}_{5}$ precipitation from a $1 \% \mathrm{Cu}$ solid solution to be about +0.004 . The resultant stress generated in the electrodeposit as a result of the IMC precipitation is then on the order of $-95 \mathrm{MPa}$ (compressive). This, of course, assumes that $\mathrm{Cu}_{6} \mathrm{Sn}_{5}$ forms from the solid solution after the deposit has been formed.

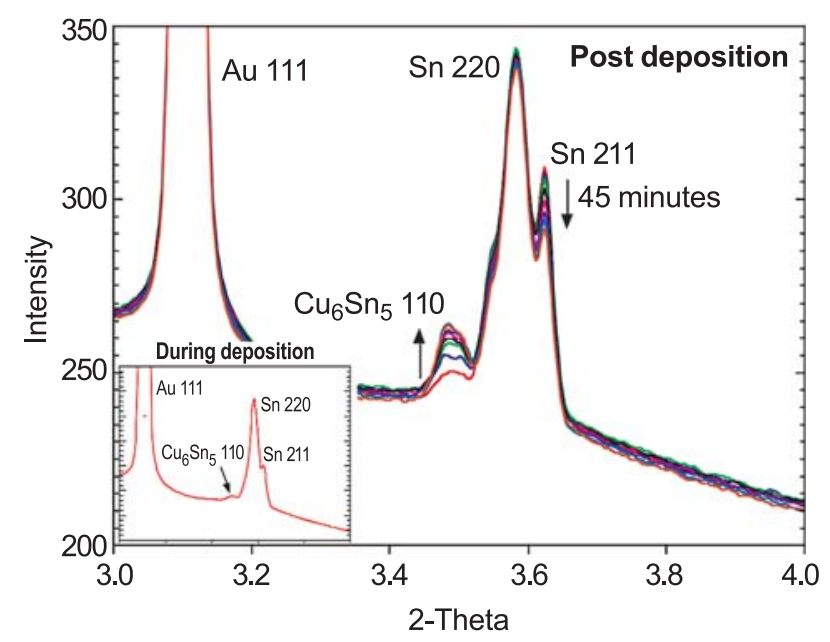

Figure 1: In situ $x$-ray diffraction $(\lambda=0.127 \AA)$ showing growth of the $\mathrm{Cu}_{6} \mathrm{Sn}_{5} 110$ reflection in the 45 minutes following deposition of bright $\mathrm{Sn}$-Cu alloy from commercial methanesulfonate electrolyte.

Figure 1 shows a series of $\mathrm{x}$-ray diffraction patterns that were taken at the Advanced Photon Source, using synchrotron radiation, during and after the deposition of a $\mathrm{Sn}-\mathrm{Cu}$ alloy onto a tungsten substrate. The inset pattern was taken during the 2-minute deposition while the remaining patterns were taken 3 minutes apart after deposition was complete. The data show that although a small amount of $\mathrm{Cu}_{6} \mathrm{Sn}_{5}$ is present when deposition is stopped (inset), the bulk of the IMC forms in the 45 minutes following deposition. Although the $\mathrm{Sn}$ reflections decrease as the IMC forms, the peaks do not change position indicating that the lattice volume of the $\mathrm{Sn}$ is constant. This supports the assumption that interstitial $\mathrm{Cu}$ does not alter the lattice volume of bet $\mathrm{Sn}$. These results indicate that IMC formation occurs after deposition is complete and is a likely cause of the higher compressive stress observed in $\mathrm{Sn}-\mathrm{Cu}$ alloy deposits.

\section{Contributors and Collaborators}

J.E. Guyer, C.E. Johnson, M.E. Williams (Metallurgy Division, NIST); M.D. Vaudin (Ceramics Division, NIST); D.R. Robinson, D. Wermeille (Advanced Photon Source, ANL) 


\section{Lead-Free Solders and Solderability}

Solders and solderability are increasingly tenuous links in the assembly of microelectronics as a consequence of ever-shrinking chip and package dimensions and the international movement toward environmentally friendly lead-free solders. In collaboration with the NEMI Pb-Free Assembly Project, we are providing the microelectronics industry with measurement tools, data, and analyses that address national needs in the implementation of lead-free solders.

\section{Ursula Kattner and Carol Handwerker}

Since 1999, NIST has served a major role in National SElectronics Manufacturing Initiative (NEMI) projects to assist the microelectronics industry implement $\mathrm{Pb}$-free solders. NEMI is an industry-led consortium of approximately 65 electronics manufacturers, suppliers and related organizations brought together to facilitate leadership of the North American electronics manufacturing supply chain. This move toward $\mathrm{Pb}$-free solders is a direct result of the European Union ban, starting in 2006, on Pb-containing solders in electronic products.

NEMI and NIST have worked together to respond to the identified needs by:

1) Identifying and providing the most important lead-free solder data for the microelectronics community (including the definitive database on thermodynamic properties of $\mathrm{Pb}$-free alloys), providing an analysis of the mechanical behavior of $\mathrm{Sn}-\mathrm{Ag}-\mathrm{Cu}$ alloys, and disseminating such data on the NIST website (see "Delivery of Thermodynamics and Kinetic Data" in this volume).

2) Developing and widely disseminating a Recommended Practice Guide on Test Procedures for Developing Solder Data.

3) Providing a list of literature references on alloys, processing, reliability, environmental issues, and components for the implementation of lead-free solders.

4) Completing the microstructure-based failure analysis on all thermally cycled assemblies as part of the NEMI project's full-scale reliability trials.

5) Providing chapters on the materials science of $\mathrm{Pb}$-free solder alloys for inclusion in four separate books on $\mathrm{Pb}$-free solder implementation.

Although the NEMI Pb-Free Assembly Project ended in 2003, in 2004 we continued to serve as an information resource to the microelectronics industry on issues related to the effects of alloy composition, reflow temperature and furnace profiles for best melting and solidification behavior of solders when circuit boards are assembled, and to the effects of $\mathrm{Pb}$ contamination on alloy melting and assembly reliability. This activity culminated in a Circuits Assembly article by Alan Rae, VP of Technology for Cookson Electronics, and Carol Handwerker of NIST on the range of acceptable alloy compositions in the $\mathrm{Sn}-\mathrm{Ag}-\mathrm{Cu}$ system. The following excerpt gives the NEMI position, and effectively the U.S. position, on the acceptable composition ranges for the new international standard $\mathrm{Pb}$-free alloy and allows both the U.S. and the Japanese preferred alloy compositions based on analyses of NIST thermodynamic data:
Although the composition of some of the alloys being commercialized varies slightly from the NEMI composition, the NEMI alloy is representative of the acceptable range of lead-free solders. Tin-silver- copper formulations with silver content between 3.0 [mass] $\%$ and $4.1 \%$ and copper between $0.5 \%$ and $1.0 \%$ are virtually indistinguishable in terms of melting point and process features. The NEMI alloy provides a model system for industry that is well characterized, and several NEMI members currently are using the alloy in production. The focus on a single lead-free alloy has helped to accelerate industry convergence on standard solder formulations, manufacturing processes and, ultimately, the timely and cost-effective conversion to lead-free assembly.

"The NIST group performed a significant service to industry by being the focal point for development of a reliable technology base to support the choice of a new lead-free alloy. They also led the way for further work on issues such as tin whiskers, which emerged from the initial lead-free work. NIST provided not only a strong technical basis but, by verifying reliability and comparing alternatives, enabled industry to choose an alloy based on extensive investigation."

\section{Dr. Robert C. Pfahl, Jr., Director International and Environmental R\&D, Motorola, (retired), and Vice President Operations, NEMI, (current)}

\section{Contributors and Collaborators}

W.J. Boettinger, K.W. Moon, T. Siewert, D. McCowan, L.C. Smith, S.W. Claggett, M.E. Williams (NIST); J.P. Clech (EPSI, Inc.); J. Bath (Solectron); R. Gedney (NEMI); E. Bradley (Motorola); J.E. Sohn (NEMI, formerly Lucent); A. Rau (Cookson Electronics); E. Benedetto (HP); R. Charbonneau (StorageTek) 


\section{Micrometer-Scale Reliability: Solder Reliability}

The electronics industry is replacing lead-tin eutectic solders with lead-free solders because of the environmental hazards of lead. In doing so, they have created a need for material property data of the new lead-free solder compositions. A test method has been developed to measure the mechanical properties using specimens on the same size scale as the solder structures used in industry.

\section{Timothy P. Quinn}

\section{Background}

Tndustry groups have pointed out the need for material property data for the new lead-free compositions of solder; this includes pooling the data that already exist and filling the critical holes in the data, especially at low-strain rates.

Most of the data that are available for all solders come from specimens that are very large (on the order of millimeters) compared to the solder structures themselves (on the order of hundreds of micrometers). As the solder structures (solder balls for flip chip packages, for example) become smaller $(\sim 150 \mu \mathrm{m})$, their dimensions approach the dimensions of the phases in the solder itself. The assumption of a homogeneous material used to analyze the stresses in the structure is challenged. We have therefore started testing samples that are on the same size scale as current solder structures to study this interaction.

\section{Accomplishments}

A test method that used "miniature" specimens was developed to mimic the size of typical soldered joints. The purpose was to examine the effects of the size of the specimens (and hence the typical structures found in industry) and to fill in the gaps in the data for the lead-free solders. Solder was cast in a Ti mold between two copper blocks, and $300 \mu \mathrm{m}$ specimens were cut from the blocks. The usable gauge length of the specimens was about $300 \mu \mathrm{m}$. We can consistently make specimens with a known thermal cycle and maintain well-defined microstructure. Because the samples were sliced off a relatively large "loaf," a large number of samples could be made in a short time.

After determining the geometry of the gage section using an optical microscope, the specimens were imaged in an ultrasonic microscope with a beam diameter of $45 \mu \mathrm{m}$ at an operating frequency of $100 \mathrm{MHz}$.

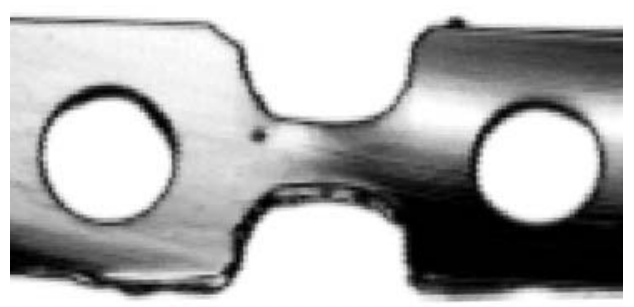

Figure 1: An ultrasound scan of a solder tensile specimen. The image was made by mapping the amplitude of the reflection off the front interface (white= larger amplitude).

Measuring the time-of-flight from the front to the rear of the sample allowed us to calculate Young's modulus knowing the density and Poisson's ratio (Figure 1).

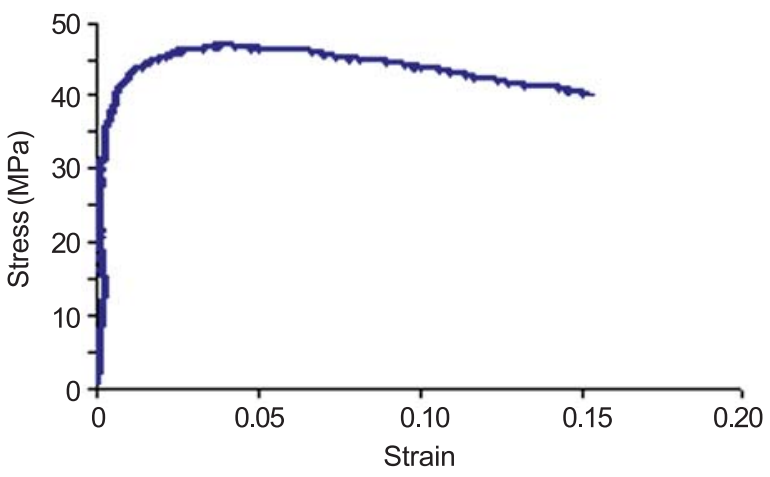

Figure 2: The measured stress-strain curve for a $\mathrm{Sn}-3.8 \mathrm{Ag}-0.7 \mathrm{Cu}$ solder.

The samples were then tested in uniaxial tension using pin-style grips. The specimens were first painted white, and then a dark line was painted on the specimen over the gauge section. A video-microscope recorded images of the gauge section at about $0.75 \mu \mathrm{m} / \mathrm{pixel}$. The edges of the gauge section were then tracked with an automated algorithm and used to calculate strain. The stress-strain curve was then plotted (for example see Figure 2). Note that because of the small displacements in the elastic region, the camera does not have enough resolution to adequately predict Young's modulus. The ultimate tensile strength, yield strength and percent elongation were all obtained using the camera data. Tests have been conducted using Sn-3.8Ag-0.7Cu, Sn-3.7Ag, Sn-0.7Cu, Sn-5Sb, and $\mathrm{Sn}-37 \mathrm{~Pb}$.

\section{Contributors and Collaborators}

I. Makover, T.P. Quinn, K.R. Waters (Materials Reliability Division, NIST); Y. Rosenthal (Ben-Gurion University, Israel) 


\section{Micrometer-Scale Reliability: Mechanical Behavior of Thin Films}

\begin{abstract}
Measurement methods and materials data are needed for the design of interconnect structures in high-performance integrated circuits. These micro- and nanometer-scale thin films are formed by physical vapor deposition; their microstructures, and hence their mechanical properties, are quite different from those of bulk materials of the same chemical composition. The ultimate goals of this project are to characterize the exact materials used in IC fabrication, at their proper size scale, and to understand the relevant deformation and failure mechanisms.
\end{abstract}

\section{David T. Read}

$\mathrm{I}^{\mathrm{n}}$ nterconnect structures in advanced integrated circuits carry power, signals, and heat from the transistors to the outside environment. These structures consist of multiple layers of thin films of conductors and dielectrics with barrier and adhesive layers. These thin films are an essential component of all advanced electronic devices, and similar materials are used in a variety of other applications, such as reflective coatings. Industry is aggressively pressing new materials into service, reducing the size of the structures, and requiring more functionality, including mechanical functionality, from all components of their structures. Design of reliable structures relies on quantitative numerical modeling, which requires accurate material property data. Because the films are formed by physical vapor deposition, their microstructures, and hence their mechanical properties, are quite different from those of bulk materials of the same chemical composition.

The objectives of this project are:

- To develop experimental techniques to measure the mechanical properties of thin films, in specimens fabricated and sized like materials used in actual commercial devices;

- To relate thin-film mechanical behavior to microstructure;

- To extend test techniques from their present level (1 $\mu \mathrm{m}$ thick, $10 \mu \mathrm{m}$ wide) to smaller specimens that are similar in size to the conductive traces used in contemporary VLSI circuits (widths of 0.1 to $1 \mu \mathrm{m}$ ).

In previous years we reported properties of aluminum, polyimide, and polysilicon films at room temperature. We have now measured the tensile properties of aluminum films from a commercial source, and lab-made electrodeposited copper films from room temperatures up to $150{ }^{\circ} \mathrm{C}$. The mechanical properties decline only slightly with increasing temperature, in a pattern normal for face-centered-cubic metals.

Microtensile Young's (elastic) modulus data for nickel were contributed to a comparison study. The microtensile value was $177 \pm 8 \mathrm{GPa}$ (most probable uncertainty). Details are given elsewhere, but the conclusion from the full set of measurements is that both the modulus and the density of this sputtered nickel film are lower than bulk values. Microtensile modulus measurements, in particular, have been suspect because the results are often lower, by $10 \%$ or more, than bulk values. See the Molecular Dynamics page of this report for more on this.

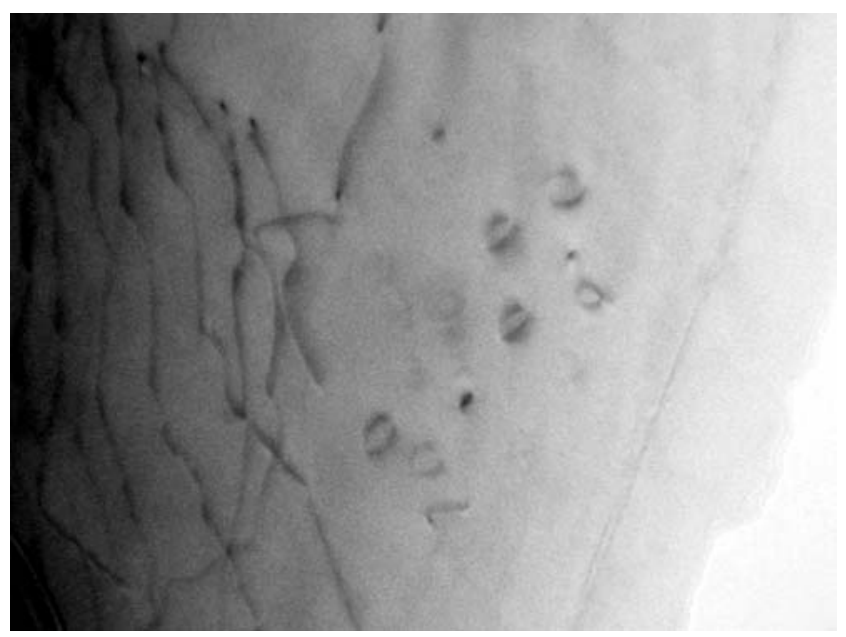

Figure 1: TEM of fractured tether of aluminum contact metal microtensile specimen. The dark lines on the left are dislocations; note their absence toward the right, near the fracture edge.

The transmission electron microscope (TEM) micrograph in Figure 1 shows two different deformation mechanisms for a thin aluminum film. Prismatic vacancy loops, seen as circular features about $20 \mathrm{~nm}$ in diameter on the right, are formed in the absence of dislocations in the thinner part of the deformation zone near the fracture edge (far right, lower). Dislocations are seen only in the thicker regions, at the left in the image. The size and density of the loops seems to be dependent on the speed of the deformation, with smaller loops $5-10 \mathrm{~nm}$ in diameter seen in more slowly deformed material.

\section{Contributors and Collaborators}

Y-W. Cheng, J.D. McColskey, R.R. Keller, R. Geiss, J. Wright (Materials Reliability Division, NIST); MOSIS Service (specimen fabrication) 


\section{Physical Properties of Thin Films and Nanostructures: Grain Size Effects on Actuator Fatigue}

\begin{abstract}
Several materials are now produced commercially with particle sizes on the order of 10-100 $\mathrm{nm}$. The availability of ceramic materials in this size classification is particularly important for the electronic component industry. Nanopowders can enable significant decreases in internal layer thickness (for multilayer devices), minimize the size and prevalence of processing defects, and boost the overall performance of components ranging from capacitors to resistive gas sensors. Quantifying these performance enhancements and their dependence on microstructure is a key objective in the Materials Reliability Division.
\end{abstract}

\section{Stephanie A. Hooker}

M any multilayer ceramic devices can benefit from the use of ultra-fine powders, including passive electronic components, gas sensors, and miniature actuators. However, fabrication issues associated with processing these high-surface-area powders have hindered their commercialization. Key challenges have included nanopowder reproducibility, optimization of processing slurries, and control of sintering profiles to produce the desired micro/nanostructure characteristics. Moreover, in many cases, predicting the optimum microstructure features for maximum performance is quite difficult, and extensive experimentation remains necessary.

One specific component that can benefit from the use of nanopowders is the multilayer piezoelectric actuator. These devices generate precise, controlled motion for translating optics, damping vibrations, redirecting air flow, and dispensing fluids. Each component consists of many active layers, the thickness of which determines the device's power requirements. Layer thickness is typically on the order of several hundred microns for conventional actuators and is driven by the need for many individual piezoelectric grains to span the active layer, thereby reducing the chance for electrical failure. Reducing grain sizes from micrometer to submicrometer scales enables dramatic decreases in layer thickness, reducing power needs and increasing applicability.

However, at issue is the piezoelectric response and, in particular, the long-term fatigue resistance. Fatigue is the change in polarization or displacement over time and is a recognized problem for state-of-the-art actuators. Because processing-induced defects tend to be on the scale of the starting powder, nanopowders can improve mechanical durability. However, a corresponding decrease in ferroelectric response is also anticipated as grain size reduces. Fatigue is affected by both the mechanical and electrical responses, making its prediction quite difficult for fine-grained components.

In FY04, we investigated the fatigue behavior of 14 microstructures obtained by controlled sintering of 80-nm PZT-5A powders. The devices tested were 1206 -sized $\left(0.30 \mathrm{~cm} \times 0.15 \mathrm{~cm}\right.$, or $\left.0.12^{\prime \prime} \times 0.06^{\prime \prime}\right)$ chips, each with 10 active layers (50 $\mu \mathrm{m}$ thick). Characterization included dielectric, impedance, and ferroelectric properties, as well as short- $(<1$ million cycles) and long-term (1-100 million cycles) degradation under combined electrical and mechanical influences.

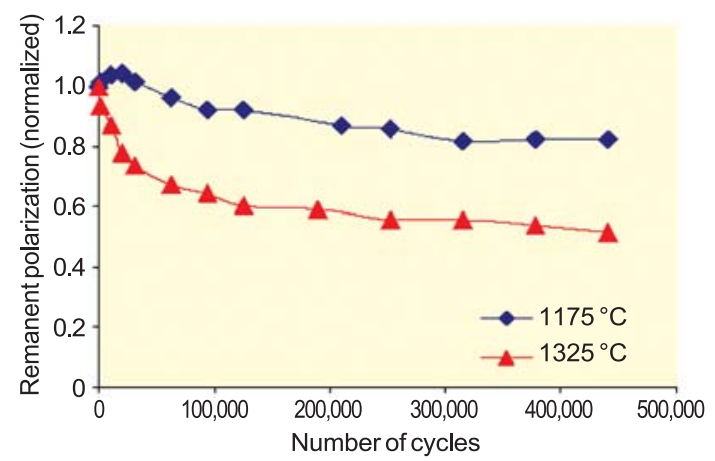

Figure 1: Effect of sintering temperature on ferroelectric fatigue.

The results clearly demonstrate the dependence of fatigue on microstructure. Figure 1 compares changes in remanent (i.e., permanent) polarization over time when devices were cycled using a $35 \mathrm{~Hz}$ sinusoidal wave with an amplitude of $+/-75 \mathrm{~V}$. The average decrease in polarization for the finest-grained materials (sintered at $1175^{\circ} \mathrm{C}$ ) was less than $20 \%$ after 0.5 million cycles, compared to nearly $50 \%$ for larger-grained components.

In FY05, we will expand on this work and examine the corresponding effects on displacement and domain reorientation for devices with even thinner internal layers. The resulting data will then be used to validate the suitability of these components for biomedical and smart structures applications, both of which demand high performance and long operational lifetimes.

\section{Contributors and Collaborators}

R. Geiss (Materials Reliability Division, NIST); C. Kostelecky, D. Deininger, K. Womer (Synkera Technologies) 


\section{Experimental and Theoretical Influence of Thin Film Texture on Ferroelectric Hysteresis}

\begin{abstract}
Commercial ferroelectric thin film devices are rapidly approaching the same nanoscale dimensions as individual grains, yet little is known about the influence of grain boundaries and local texture on ferroelectric properties. Accordingly, several complementary experimental and theoretical techniques are being developed to assess the local ferroelectric response. By combining quantitative piezoelectric force microscopy (PFM), electron backscattering diffraction (EBSD), and two-dimensional object oriented finite-element modeling (OOF), various individual crystallographic distributions with either beneficial or detrimental effects can be identified and eventually predicted.
\end{abstract}

\section{Bryan D. Huey, R. Edwin García and John E. Blendell}

Crystallographic orientation can now be determined with nanometer scale lateral resolution using the newly installed electron backscattering diffraction system (EBSD), allowing the orientation of individual ferroelectric grains to be mapped as indicated in Figure 1a.
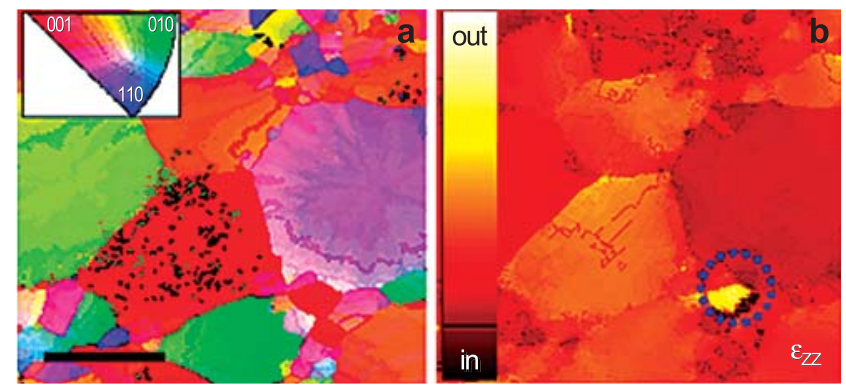

Figure 1: (a) Measured surface orientation of PZT thin film and (b) predicted strain normal to the surface for the same grains when biased with an electrode as indicated. Bar $=20 \mu \mathrm{m}$.

Recent advances in two-dimensional (2-d) object oriented finite element modeling (OOF) have made it possible to predict the piezoelectric response of a polycrystalline thin film with full consideration of the sample texture as determined by EBSD. Figure $1 \mathrm{~b}$ demonstrates the strain normal to the surface for an applied bias of $1 \mathrm{~V}$ across a $100 \mathrm{~nm}$ thick film using a $100 \mathrm{~nm}$ diameter electrode, where the electrode is positioned across multiple grains of differing orientations. The local response is strongly dependent on texture, in some cases oppositely oriented.

Piezoelectric force microscopy (PFM) was employed to determine experimentally the ferroelectric

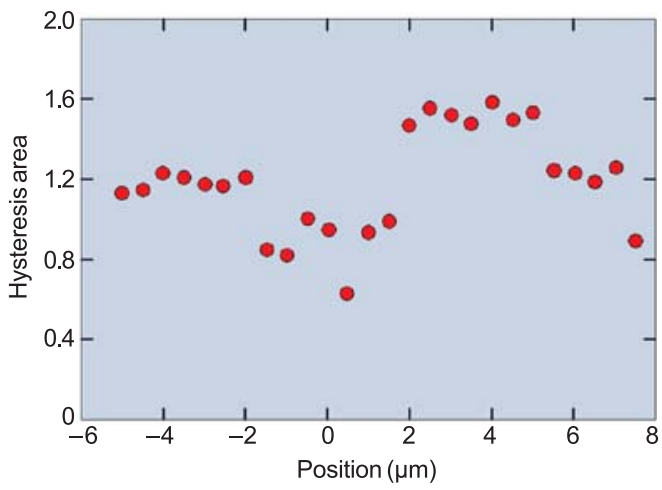

Figure 2: Measured piezoelectric hysteresis areas of several grains/boundaries.

properties of the same grain and grain boundary regions considered by EBSD and OOF. This AFM-based technique is widely used, but can be significantly hindered by several instrumental artifacts that were identified and overcome during this work. The area of piezoelectric hysteresis loops determined using the newly developed quantitative methods is indicated in Figure 2 for several distinct grains and boundaries. The measured hysteresis variations correlate with grains and grain boundaries of differing misorientation as measured by EBSD and correlates with strains calculated by OOF.

Key accomplishments and conclusions include:

1) Novel methods for identifying and overcoming artifacts in piezoelectric force microscopy to allow quantitative local piezoelectric hysteresis measurements;

2) Unique 2-d simulations of converse-piezo actuation with complete incorporation of true thin-film texture;

3) The importance of uniform texture for optimal ferroelectric properties when device dimensions approach the lateral grain size; and

4) The likelihood of domain pinning or even crack initiation at certain grain boundaries and intersections as a result of local orientation.

Results from this work were presented at the SPIE Ferroelectrics conference, American Ceramic Society annual meeting, European Congress on Nanoscale Materials (Nano-04), IEEE Ferroelectrics and Ultrasonics joint meeting, and several invited seminars at NIST and various universities. Publications in the Journal of the American Ceramic Society, Journal of Electroceramics, and Journal of Applied Physics are under review.

\section{Contributors and Collaborators}

M.D. Vaudin, E.R. Fuller, Jr. (Ceramics Division, NIST); S. Hong (Samsung) 


\section{Organic Electronics}

The field of organic electronics has dramatically emerged in recent years as an increasingly important technology encompassing a wide array of devices and applications including embedded passive devices, flexible displays, and sensors. Device performance, stability, and function critically depend upon charge transport and material interaction at the interfaces of disparate materials. We develop and apply non-destructive measurement methods to characterize the electronic and interfacial structure of organic electronics materials with respect to processing methods, processing variables, and materials characteristics.

\section{Eric K. Lin and Jan Obrzut}

$\mathrm{O}$ ganic electronic devices are projected to revolutionize integrated circuits through new applications that take advantage of low-cost, highvolume manufacturing, nontraditional substrates, and designed functionality. The current state of organic electronics is analogous to the early stages of the silicon electronics industry with the concurrent development of multiple material platforms and processes, and a lack of measurement standardization between laboratories. A critical need exists for new diagnostic probes, tools, and methods to address new technological challenges.

Organic electronics presents fundamentally different measurement challenges from those identified for inorganic devices. The adoption of this technology will be advanced by the development of an integrated suite of metrologies to correlate device performance with the structure, properties, and chemistry of materials and interfaces. We are developing measurement methods to provide the data and insight needed for the rational and directed development of emerging materials and processes.

This year, we have addressed three areas covering a spectrum of active organic electronic materials: dielectric measurements for embedded passive devices,
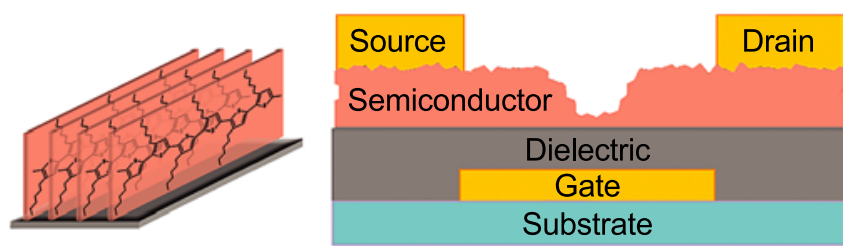

Figure 1: Schematic diagrams of the local structure of poly 3-hexylthiophene, an organic semiconductor, near an interface (left) and the architecture for an organic transistor (right). measurements of moisture permeation rates through barrier films for organic light-emitting diode displays, and measurements of the orientation and structure of organic semiconductors for transistor applications.

To meet the metrology needs for embedded passive devices, two new measurement methods were successfully developed and will find widespread use within industry (see Highlight). A NIST-developed Test Method for Dielectric Permittivity and Loss Tangent of Embedded Passive Materials from $100 \mathrm{MHz}$ to $12 \mathrm{GHz}$ was accepted and recommended as a standard test method. In addition, a new test was developed and applied for testing passive materials at high electric fields and voltages.

For organic light-emitting diode displays, a key measurement challenge is the quantification of the moisture permeation rates through a barrier coating. Any moisture able to penetrate the coating compromises the performance of the device. There is a need to measure moisture permeation rates as low as $10^{-6} \mathrm{~g} / \mathrm{m}^{2} \mathrm{~d}$. In collaboration with Vitex Systems, Inc., neutron and $\mathrm{x}$-ray reflectivity measurements demonstrated the capability of measuring these low permeation rates in addition to providing spatial detail about the transport of water within the barrier material itself.

Finally, near-edge x-ray absorption fine structure (NEXAFS) spectroscopy was applied to several classes of organic electronics materials to investigate the electronic structure, chemistry, and orientation of these molecules near a supporting substrate. NEXAFS provides a powerful and sensitive method to probe the interfacial structure that is critical to the performance of these devices. For example, it was found that several organic semiconductor molecules preferentially align "edge-on" rather than "face-down" on solid substrates.

\section{Contributors and Collaborators}

D. DeLongchamp, O. Anopchenko, K. Kano, B. Vogt, H. Lee, E. Jablonski, W. Wu (Polymers Division, NIST); S. Sambasivan, D. Fischer (Ceramics Division, NIST); L. Richter (CSTL); C. Richter, E. Vogel (EEEL); D. McGregor, G.S. Cox, J. Felten (DuPont); D. Fritz (MacDermid); T. Bergstresser (Gould Electronics); K. Fjeldsted (Electro Scientific Industries); R. Crosswell (Motorola); C. Vanderpan (UL); R. Whitehouse (SanminaSCI); L. Moro, N. Rutherford (Vitex); V. Subramanian (U.C. Berkeley); Z. Bao (Stanford University); B. Ong (Xerox); A. Afzali (IBM); G. Jabbour (Arizona State University); R. Pilston (Plextronics); Y. Jung, D. Yoon (Seoul National University) 


\section{Combinatorial/Phase Diagram Approach for Metallization to Wide-Band-Gap Semiconductors}

\begin{abstract}
The development of wide-band-gap semiconductor optoelectronic and electronic devices is hindered by poor electrical contact performance. Reliability issues for contacts include the requirements to be low-resistance, morphologically smooth and thermally stable. This project develops a strategy to improve electrical and morphological characteristics of Ohmic contacts to GaN thin films by optimizing metallization schemes using an integrated phase diagram/combinatorial approach.
\end{abstract}

\section{Albert V. Davydov and William J. Boettinger}

$\mathrm{T}$ $\mathrm{i} / \mathrm{Al} / \mathrm{Ti} / \mathrm{Au}$ metallizations are the most commonly used electrical contact to n-type GaN-based device structures. However, the overall composition (i.e., thickness ratios) and the thermal processing of metal layers are not yet optimized. A rationale for designing the optimum metallization scheme also has not been developed. This work demonstrates that the phase diagram approach, along with a combinatorial experimentation method, is a useful tool in the design and optimization of electrical contacts to $\mathrm{GaN}$ and other wide-band-gap semiconductors.

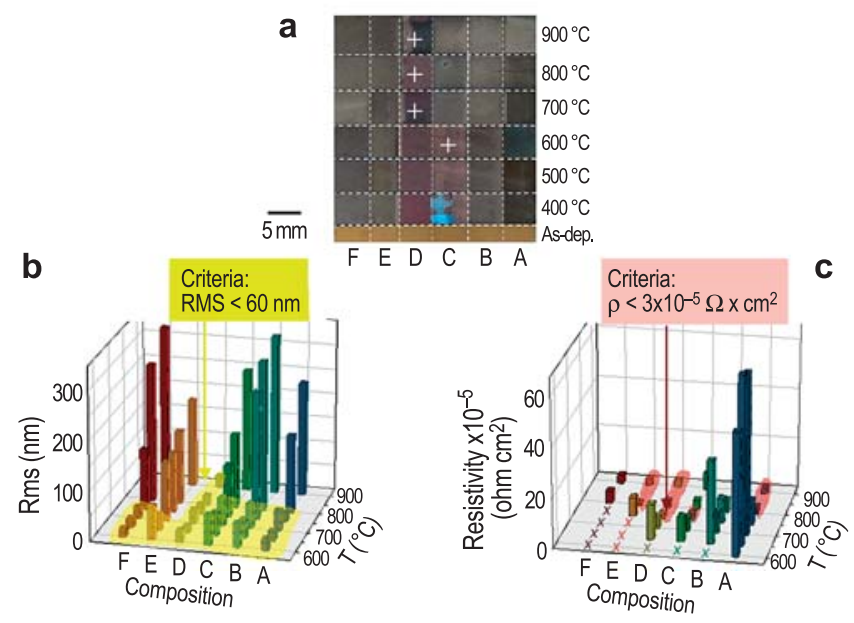

Figure 1: a) 36-element combinatorial library of annealed Ti/Al/Ti/Au contacts on $n$-GaN: metal compositions change along the $x$-axis from $A$ to $F$, annealing temperature changes from $600^{\circ} \mathrm{C}$ to $900^{\circ} \mathrm{C}$ along the $y$-axis; " $\boldsymbol{X}$ "s mark the library elements with the lowest resistivity and smoothest morphology; b) map of surface roughness of the combinatorial array: yellow-shaded area represents the contacts with the smoothest $(\mathrm{rms}<60 \mathrm{~nm})$ morphology; c) map of electrical resistivity of the combinatorial array: red-shaded area represents the contacts with the lowest $\left(\rho<3 \times 10^{-5} \Omega \times \mathrm{cm}^{2}\right)$ resistivity.
A combinatorial library of $\mathrm{Ti} / \mathrm{Al} / \mathrm{Ti} / \mathrm{Au}$ metallization was prepared and characterized electrically and microstructurally in order to establish optimum composition and processing parameters for the realization of high-quality Ohmic contacts. An array of metallic elements with varying $\mathrm{Ti} / \mathrm{Al} / \mathrm{Ti} / \mathrm{Au}$ thicknesses was deposited by combinatorial ion-beam sputtering (CIBS) on $\mathrm{n}-\mathrm{GaN} / \mathrm{c}$-sapphire substrate followed by rapid-thermal annealing (RTA) in the $600{ }^{\circ} \mathrm{C}$ to $900^{\circ} \mathrm{C}$ temperature range. The library design of metal compositions and RTA temperatures was guided using the $\mathrm{Al}-\mathrm{Au}$-Ti phase diagram so that various $\mathrm{Ti} / \mathrm{Al} / \mathrm{Ti} / \mathrm{Au}$ contact compositions represented different regions on the phase diagram.

Four-probe transmission-line-method and Hall electrical measurements were used to assess contact resistivity and $\mathrm{GaN}$ sheet resistance. X-ray diffraction, white-light interferometric microscopy, atomic-force microscopy, field-emission scanning-electron-microscopy, and transmission-electron-microscopy were used to evaluate the microstructure and morphology of contacts and the metal/GaN interfaces. The most Au-rich/Ti-poor metallization (E in Figure 1a), $\operatorname{Ti}(20 \mathrm{~nm}) / \mathrm{Al}(85 \mathrm{~nm}) /$ $\mathrm{Ti}(15 \mathrm{~nm}) / \mathrm{Au}(150 \mathrm{~nm})$ with an RTA of $800{ }^{\circ} \mathrm{C} / 30 \mathrm{~s}$ in argon, produced the lowest $\left(\rho=1.3 \times 10^{-5} \Omega \times \mathrm{cm}^{2}\right)$ contact resistivity with reasonable $(\mathrm{rms}<150 \mathrm{~nm})$ surface morphology. However, the most Al-rich metallization (D in Figure 1a), $\operatorname{Ti}(20 \mathrm{~nm}) / \operatorname{Al}(170 \mathrm{~nm}) / \operatorname{Ti}(5 \mathrm{~nm}) /$ $\mathrm{Au}(50 \mathrm{~nm})$ with RTA of $750^{\circ} \mathrm{C} / 30 \mathrm{~s}$ in argon, produced superior surface morphology $(\mathrm{rms}=20 \mathrm{~nm})$ and comparable resistivity of $\rho=2.2 \times 10^{-5} \Omega \times \mathrm{cm}^{2}$. It is suggested that scheme (E) with the Au-rich contact can be used for operating in oxidizing environments, where thick Au top layer will serve as a protective cap layer, while scheme (D) can be suitable for applications where contact surface roughness is critical. According to XRD results, the superior surface morphology of contacts in the D-series correlates with the absence of phase transformations in the $600{ }^{\circ} \mathrm{C}$ to $900{ }^{\circ} \mathrm{C}$ temperature interval after the initial formation of $\mathrm{Al}_{2} \mathrm{Au}$ and $\mathrm{Al}_{3} \mathrm{Ti}$ phases in metal films.

The advanced metallizations developed in the project will be validated in industrial high-electron mobility transistors (HEMTs) at Northrop-Grumman Corporation.

\section{Contributors and Collaborators}

L.A. Bendersky, D. Josell, U.R. Kattner, A.J. Shapiro (Metallurgy Division, NIST); J.E. Blendell, R.S. Gates, P.K. Schenck, M.D. Vaudin (Ceramics Division, NIST); T. Zheleva (Army Research Laboratory); H.G. Henry (Northrop-Grumman); Q.Z. Xue (Intematix Corp.); A. Motayed (Howard University); I. Takeuchi (Univ. of Maryland) 


\section{Metrology and Standards for Optoelectronic Materials}

The optoelectronics industry needs measurement techniques and accurate materials property data to calibrate their deposition processes and to evaluate both prototype and finished devices. Recent work has focused on the electronic band structure of InGaAsN films (for infrared detectors and solar cells) and calibration of strain in ultrathin $\mathrm{Al}_{x} G a_{1-x} A$ s films and optical properties of $\mathrm{GaN}$ and $\mathrm{ZnO}$ nanowire structures.

\section{Albert J. Paul and Lawrence H. Robins}

$\mathrm{n}_{y} \mathrm{Ga}_{1-y} \mathrm{As}_{1-x} \mathrm{~N}_{x}$ semiconductor alloy films show promise for infrared optoelectronic devices.

Data on the electronic band structure are needed to facilitate device design and simulation. We examined Si-doped $\operatorname{In}_{y} \mathrm{Ga}_{1-y} \mathrm{As}_{1-x} \mathrm{~N}_{x}$ films with $x<0.012$ and $0.052<y<0.075$ by photoreflectance (PR) spectroscopy, supplemented by secondary ion mass spectroscopy (SIMS) and energy dispersive x-ray spectroscopy (EDS, for composition), XRD (strain), and Hall effect (electrical properties). The composition and carrier concentration dependence of the critical point energies, designated $E_{-}$(fundamental gap), $E_{-}+\Delta_{S O}$ (split-off valence band to conduction band), and $E_{+}$(valence band to nitrogen impurity band) was successfully explained by a band anti-crossing and free-carrier band-filling model with a small number of adjustable parameters.
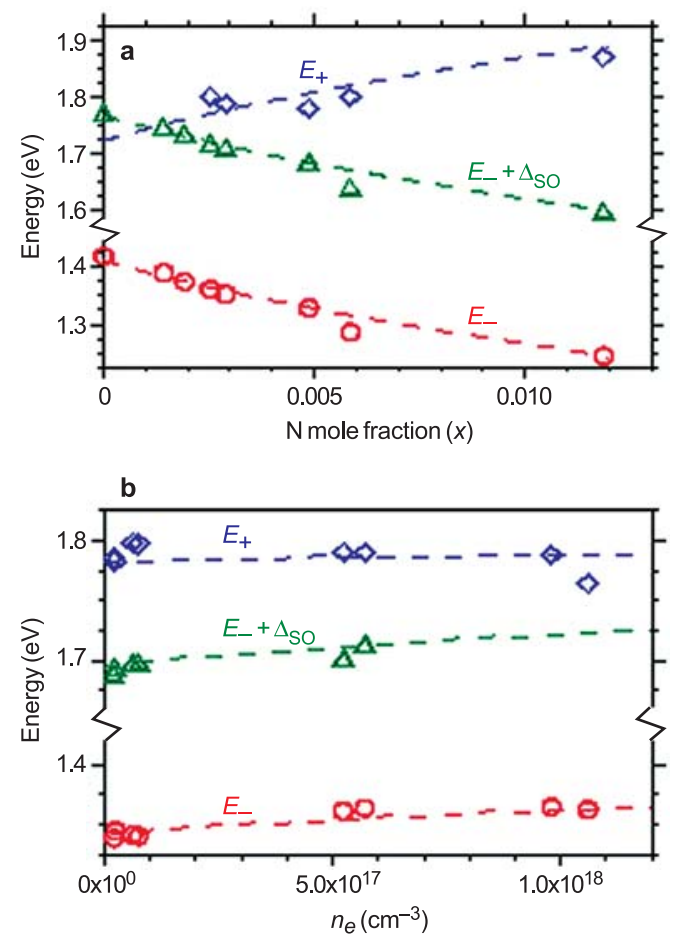

Figure 1: PR critical-point energies: data and modeling.
The measured critical-point energies (symbols) and the best-fit model (dashed curves) are plotted as functions of $\mathrm{N}$ mole fraction $(x)$ in Figure 1(a), and Hall carrier concentration $\left(n_{e}\right)$ in Figure 1(b).

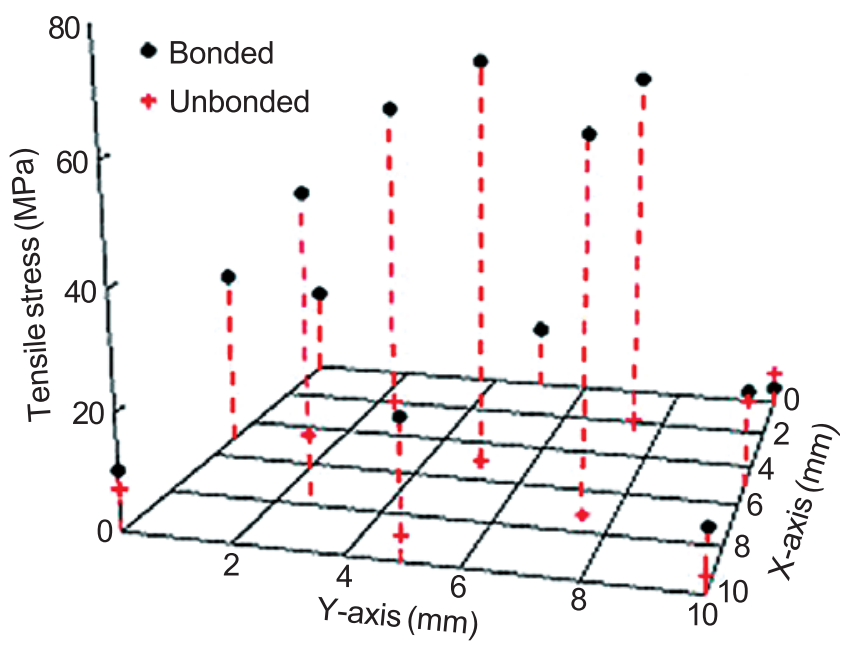

Figure 2: Tensile stress distributions in two $\mathrm{Al}_{0.2} \mathrm{Ga}_{0.8} \mathrm{As}$ structures, obtained from calibrated PL measurements.

We are continuing to develop methods for quantifying optical measurements of stress and strain in III-V semiconductors. By calibrating the shift of the Raman and PL peaks with known applied stress in a load cell, we are able to measure stresses due to differential thermal expansion, film-substrate lattice mismatch, and device packaging. As an example, the stress induced by bonding of a $10 \mathrm{~mm} \times 10 \mathrm{~mm}$ $\mathrm{Al}_{0.2} \mathrm{Ga}_{0.8} \mathrm{As}$-on-GaAs sample (grown as a composition SRM) to a stainless steel disk was measured by this technique. Results from selected locations on the bonded sample and, for comparison, an unbonded piece cut from the same wafer, are shown in Figure 2. A large tensile stress is seen to occur near the center of the bonded piece, but not in the unbonded piece.

Stress measurements of ultrathin ( $5 \mathrm{~nm}$ to $30 \mathrm{~nm}$ ) $\mathrm{AlGaAs}$ films have been initiated to compare spatially resolved measurements of stress near the edges of ultrathin films with the quantitative predictions of Object-Oriented Finite Element (OOF) modeling.

\section{Contributors and Collaborators}

E. Fuller, Y.-S. Kang, G. White (Ceramics Division, NIST); K.A. Bertness, N.A. Sanford (Optoelectronics Division, NIST); A.V. Davydov, A.J. Shapiro (Metallurgy Division, NIST); D. Chandler-Horowitz (Semiconductor Electronics, NIST); M.M.E. Fahmi, S.N. Mohammad (Howard University) 


\section{Nanomagnetodynamics}

In order to pursue the rapid development path set out for hard drives and magnetic memory, industry needs the ability to measure and control magnetization on nanometer length scales and nanosecond time scales. This project focuses on the metrology of dynamics and damping in magnetic thin films, especially on the effects of nano-scale dimensions and defects in ferromagnetic resonance measurements.

\section{Robert D. McMichael}

$\mathrm{W}$ e are developing ferromagnetic resonance (FMR) techniques to measure the static and dynamic properties of thin films and patterned arrays of technologically important ferromagnetic metals and their interfaces with normal metals. The primary results include measurements of surface anisotropy, which becomes increasingly important for nanoscale damping, which governs behavior on the sub-nanosecond time scale, and assessment of the magnetic homogeneity of thin films or patterned arrays of magnetic bits. These results are communicated to the magnetic data storage industry through conference presentations, journal articles and site visits.

For nanostructured materials, surface effects and interfacial effects are important. For example, micromagnetic calculations show that switching behavior of magnetic memory cells will be affected by surface anisotropy on the edges of the lithographically patterned elements, an effect that has not been appreciated until recently. Measuring the anisotropy of films of varying thickness with ferromagnetic resonance enables the determination of surface anisotropy values for Permalloy that will be used in the micromagnetic design of MRAM cells.

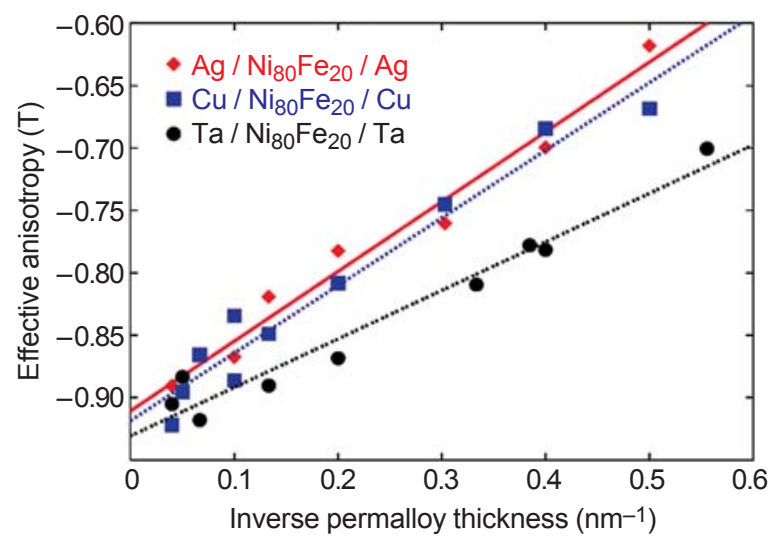

Figure 1: Surface anisotropy measurements for Permalloy films with different normal metal over/underlayers. The slopes of the fit lines correspond to surface anisotropy.
A continuing thrust of this project has been the development of models that describe broadening of the FMR linewidth by defects. These models, which are specifically designed for thin films, account for both inhomogeneity and magnetic interactions. Our earlier models were restricted to "local" defects such as anisotropy. One important application of these models is as a method for measurement of anisotropy variations and axis alignment in perpendicular recording media. This year, we have capped off the model development with a model for the non-local effects of film roughness.

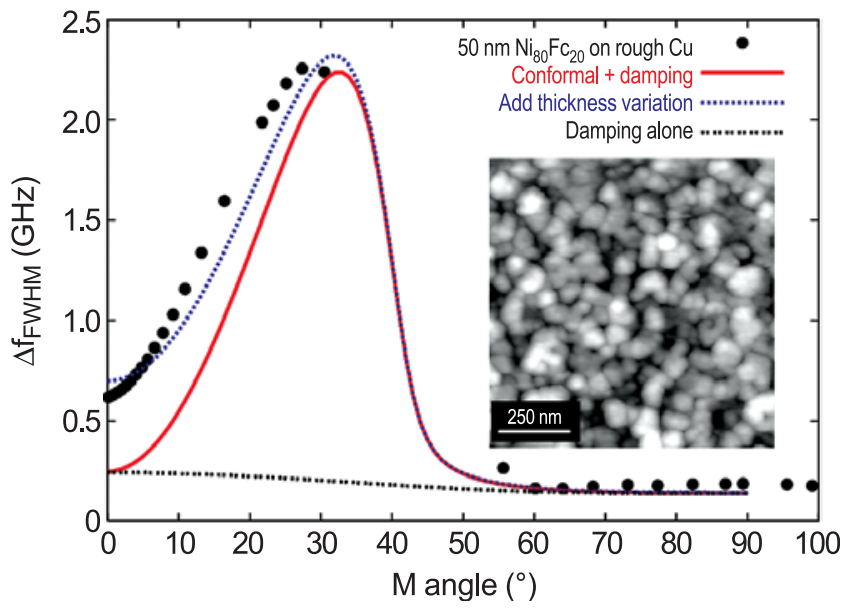

Figure 2: FMR line broadening measured in a Permalloy film and calculated almost entirely from the microstructure of the underlayer shown in the inset.

Testing of the roughness-linewidth model involved making variable roughness substrates, characterizing the roughness by atomic force microscopy (AFM), and measuring nearly conformal Permalloy overlayers in FMR. Using only the AFM microstructural data and the known properties of Permalloy, and assuming conformal roughness, the model predictions agreed extremely well with the measurements. Allowing one free parameter describing thickness variations, the agreement with the experiment is nearly perfect. Validation of this model is the first example of linewidth modeling based entirely on the measured microstructure.

\section{Contributors and Collaborators}

J.O. Rantschler, B.B. Maranville, J. Mallett, T. Moffat, W.F. Egelhoff, Jr., A.P. Chen (Metallurgy Division, NIST); J.A. Borchers, S.K. Satija (NIST Center for Neutron Research); W. Bailey (Columbia U.); A. Arrott (Virginia State University) 


\section{Electrodeposited $\mathrm{Pt}_{1-\mathrm{x}}(\mathrm{Fe}, \mathrm{Co}, \mathrm{Ni})_{x}$ Alloys}

Platinum-iron group alloys have at least two important potential applications: as a medium for magnetic recording and as $\mathrm{CO}$ tolerant catalysts in fuel cells. Alloy formation by electrodeposition presents a convenient and inexpensive alternative to conventional vacuum methods. A simple thermodynamic model has been used to account for the observed dependence of alloy composition on the deposition potential.

\section{Jonathan J. Mallett, William F. Egelhoff, Jr., and Thomas P. Moffat}

$\mathrm{P}$ erhaps the most exciting application of iron-group Pt alloys involves their high magnetic coercivity and anisotropy in the $\mathrm{L} 1_{0}$ phase. This makes them ideal candidates for future magnetic storage media, which will require high coercivity material to sustain the reductions in bit size needed to increase storage density. Controlled alloy composition and microstructure are essential for achieving the $\mathrm{L} 1_{0}$ phase, which requires close to a 50:50 composition.

Iron-group Pt alloys may also find application as poison-resistant hydrogen fuel cell catalysts. A primary concern is the lifetime and efficiency of the catalyst used to oxidize hydrogen gas in the cell. Recent research suggests that replacing the traditional Pt catalyst with a Pt alloy containing up to $15 \%$ of an Fe-group element can dramatically improve the resistance of the catalyst to $\mathrm{CO}$ poisoning. An inexpensive, reliable process for producing such alloys is, therefore, likely to be of considerable utility to fuel cell development.

An electrodeposition method has been developed that allows smooth ( $<5 \mathrm{~nm}$ RMS roughness) alloy films to be deposited to a thickness of between $20 \mathrm{~nm}$

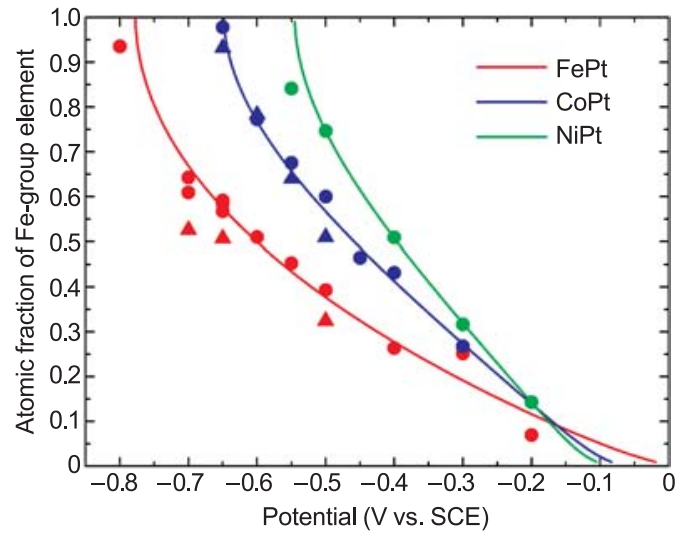

Figure 1: The theoretical (curves) and experimental (data points) dependence of composition on potential. and $3000 \mathrm{~nm}$. Deposition takes place from a bath containing $3 \mathrm{mM} \mathrm{PtCl}_{4}$ and $0.1 \mathrm{M}$ of the reactive metal ions $\left(\mathrm{Fe}^{2+}, \mathrm{Ni}^{2+}\right.$, or $\left.\mathrm{Co}^{2+}\right)$. The deposition potentials were chosen such that deposition of the reactive metal in its bulk form would have been energetically impossible. Platinum on the other hand readily deposits at all of the chosen potentials. The incorporation of the reactive metal occurs due to the highly negative enthalpy of mixing of the alloy. This reduces the activity of the reactive element in the alloy and allows its deposition to occur at more positive potentials than would be possible for the pure element. The reactive ions are in much higher concentration than the noble complexed platinum ions, which allows the former to be reduced in a kinetically facile way that enables equilibrium alloy compositions to be achieved. Under these conditions, the alloy composition and morphology was found to remain remarkably invariant to significant perturbations in constituent concentrations. The strong dependence of composition on potential is shown in Figure 1 for three alloys.

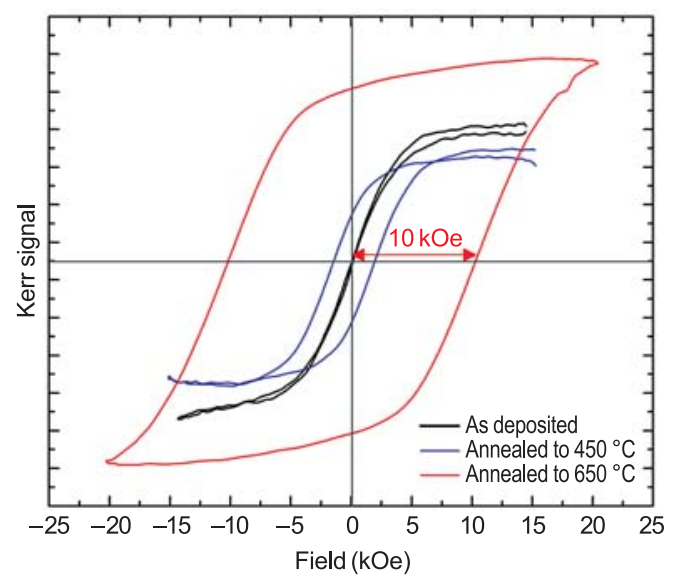

Figure 2: The increase in coercivity on annealing.

Figure 2 shows the effect of annealing an FePt film grown on a $\mathrm{Cu}(001)$ substrate. The transformation to the $\mathrm{L} 1_{0}$ phase results in a dramatic increase in magnetic coercivity. The 10 kOe coercivity produced by the $650{ }^{\circ} \mathrm{C}$ anneal is suitable for next-generation magnetic media.

Current activities are focused on extending the investigation to ternary alloys and expanding the available deposited structures to include nanoparticles (useful as catalysts) and template-formed nanostructures (for future magnetic recording media).

\section{Contributors and Collaborators}

E.B. Svedberg (Seagate Technology) 


\section{Novel Magnetic Materials for Sensors and Ultra-High Density Data Storage}

\begin{abstract}
Magnetic sensors play a central role in many important technologies ranging from health care to homeland security. A common need among these technologies is greater sensitivity and smaller size. In ultra-high density data storage, one of the most pressing needs is for nano-structured media that store data at ever-increasing densities. Improved methods for the magnetic isolation of grains in ultra-thin films are a key need; we have initiated research programs in both areas.
\end{abstract}

\section{William F. Egelhoff, Jr.}

JIST's Magnetic Engineering Research Facility 1 (MERF) is one of the most versatile facilities in the world for the fabrication and analysis of novel magnetic thin films. Two new areas of research, magnetic sensors and magnetic media, illustrate this versatility. The common link is that both require novel magnetic thin films.

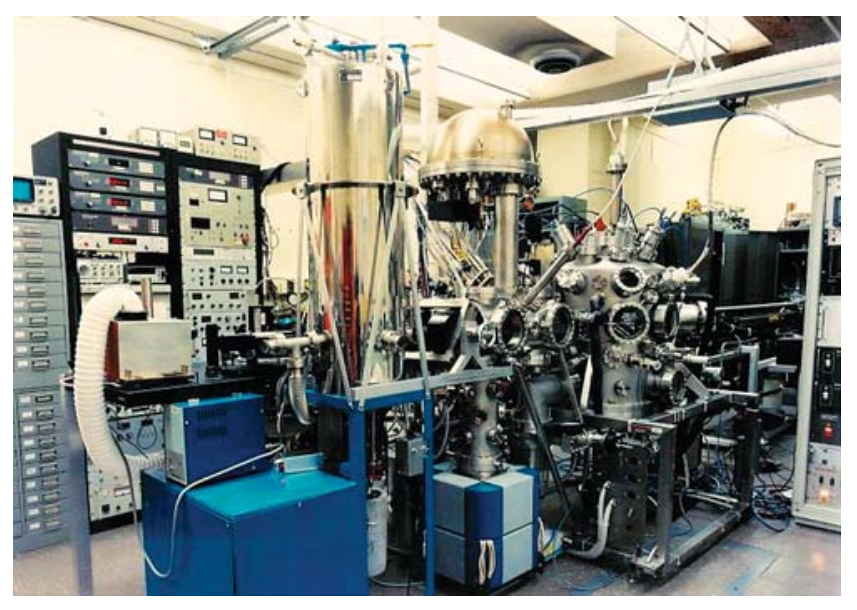

Figure 1: The Magnetic Engineering Research Facility (MERF).

It appears that ultra-sensitive magnetic sensors will require the use of thin films of ultra-soft magnetic alloys. These alloys often have a very complex composition such as $\mathrm{Fe}_{73.5} \mathrm{Si}_{13.5} \mathrm{~B}_{9} \mathrm{Nb}_{3} \mathrm{Cu}_{1}$ and require a very specific annealing process. These materials are well-known in bulk form but have never been produced in thin-film form. The project goal is to carry out the metrology needed to produce these materials as ultra-thin films.

We are presently modifying the MERF to add the capability of ion-beam sputter deposition (IBSD). IBSD, which is based on sputtering a target of the desired composition, is the only reliable method for maintaining the correct composition for complex ultra-thin films. MERF will soon become the only deposition system in the world equipped for ultra-thin film deposition by IBSD, $\mathrm{AC}$ and DC magnetron sputtering, and molecular beam epitaxy. Such versatility will allow MERF to continue to play a leading role in magnetic thin-film research.

In the area of novel magnetic media we have been collaborating closely with Seagate. Seagate is the world's leading manufacturer of hard-disk drives, and for the past two years, they have been sending a Ph.D. physicist from their research labs to work with us, first on ballistic magnetoresistance, and now on novel magnetic media.

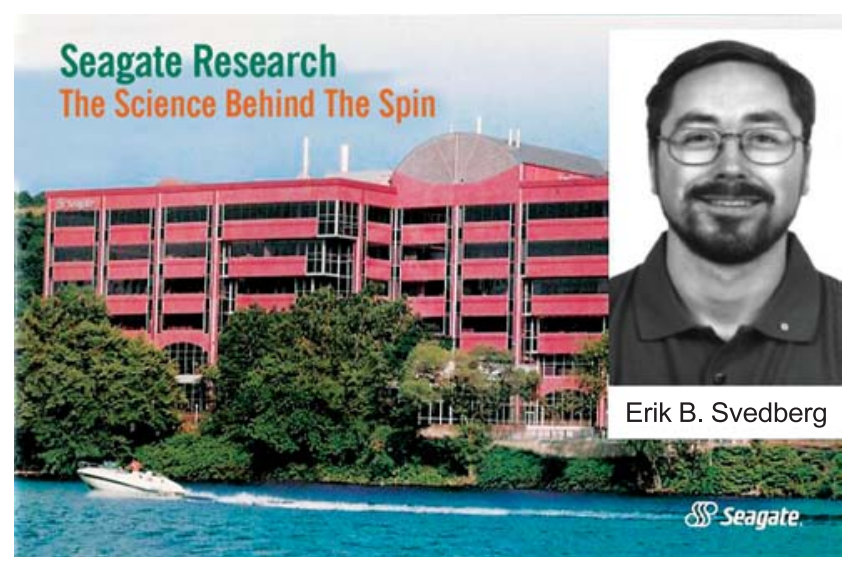

Figure 2: Close collaborations with Seagate have contributed to the success of this project.

Recent work determined a novel method for magnetically decoupling grains in CoPd media. CoPd multilayers are one of the leading candidates for the next generation of magnetic media. We have found that if a Au film is deposited on top of the CoPd and anneal in air, two unexpected phenomena occur as a result of rapid diffusion of atoms along grain boundaries. One is that Co atoms diffuse to the surface, react with oxygen, and remain at the surface. The other is that $\mathrm{Au}$ atoms diffuse into the grain boundaries replacing Co. The net effect is that the grain boundaries are demagnetized, and each grain can magnetically switch independently of its neighbors. This method provides the best magnetic isolation of grains yet found.

\section{Contributors and Collaborators}

E.B. Svedberg, D. Weller (Seagate); R.D. McMichael, T.P. Moffat, J. Mallett (Metallurgy Division, NIST); D.P. Pappas (Electromagnetics Division, NIST) 


\section{Discovery of Spin Density Waves in a Ferromagnet: Fe-Al}

Magnetism is endemic to our society. Ferromagnets are the basis for information storage in computers and recording devices; they are found in all motors, transformers, and generators; and they are used in a host of other products like credit cards, cellular telephones, radios, and televisions. However, the magnetic characteristics of the ferromagnet used in each of these applications varies with the product. Consequently, industry needs to know the interconnection between material characteristics and magnetic properties, and how to control them. A critical element in such an understanding is knowledge of the fundamentals of spin interaction, and how to measure it. NIST provides the metrology needed by industry to do this.

\section{Robert D. Shull and Jeffrey W. Lynn (NCNR)}

Tn 1962, a new type of interaction between magnetic 1 spins was predicted - a band structure effect caused by an instability in the conduction electron gas near the Fermi surface. The instability creates a new low-energy state in which the magnetic spin orientations form a standing wave, called a spin density wave (SDW). Similarly, a wave of charge, called a charge density
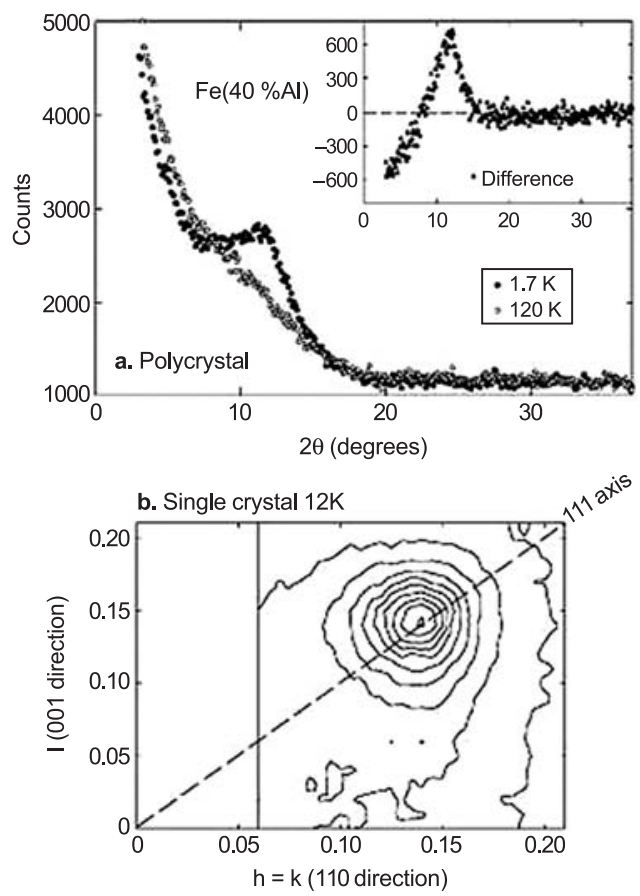

Figure 1: Neutron scattering intensity for a) polycrystalline $\mathrm{Fe}_{60} \mathrm{Al}_{40}$ at $120 \mathrm{~K}$ and $1.7 \mathrm{~K}$ with their difference in the inset and b) single crystal $\mathrm{Fe}_{60} \mathrm{Al}_{40}$ at $12 \mathrm{~K}$ shown as contours about the $(1 / 7,1 / 7,1 / 7)$ peak. wave (CDW), was also predicted. Charge density waves have since been found experimentally, and the SDW concept has been successfully used to explain the unusual incommensurate antiferromagnetism in Cr. However, evidence for SDWs in a ferromagnet has proven elusive.

Using neutron diffraction measurements at NIST, we recently discovered a SDW in a single crystal of $\mathrm{Fe}_{60} \mathrm{Al}_{40}$, a material with ferromagnetically interacting spins. This was shown (Figure 1) by the presence of scattering intensity at locations where there was no atomic reciprocal lattice vector (i.e., at places where there would normally be no coherent atomic scattering). In fact, the data showed there are several SDWs in the material with wave vectors near (110) directions (shown in Figure 2). No such waves were found along either (100) or (111) directions.

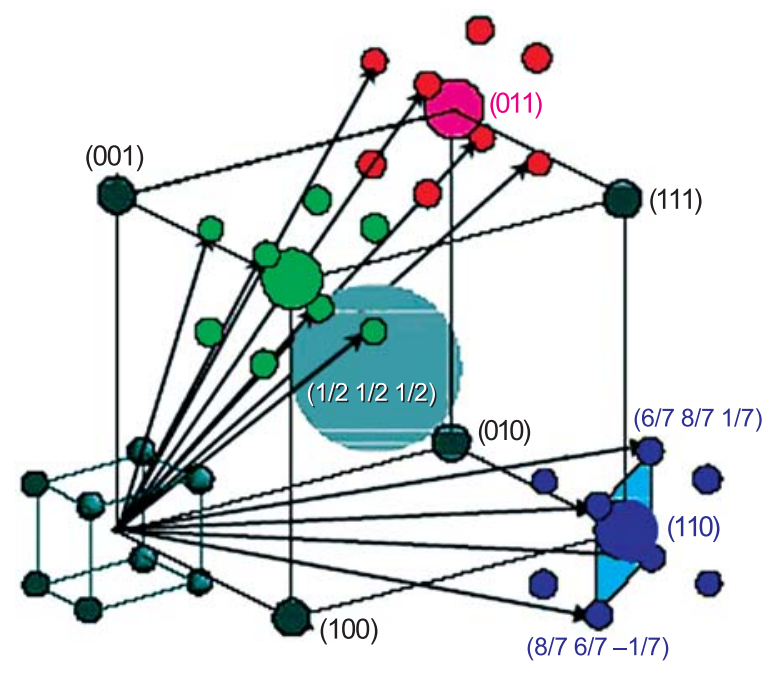

Figure 2: The reciprocal lattice spots in $\mathrm{Fe}_{60} \mathrm{Al}_{40}$, showing the $S D W$ wave vectors (black arrows). (Physical Review Letters 91, 217201-1 (2004).)

The presence of SDWs shows the exchange interactions between magnetic spins in $\mathrm{Fe}-\mathrm{Al}$ is a band structure (i.e., non-local) effect and explains the large magnetism in these materials containing a large content of "non-magnetic" atoms. This discovery should be as important to the understanding of magnetism in metals as the SDWs in Cr. In Cr, the SDW wave vector, close to a (100) direction, makes moments of nearest neighbors in the bcc structure almost oppositely aligned.

\section{Contributors and Collaborators}

A.S. Arrott, D.R. Noakes, M.G. Belk (Virginia State University); S.C. Deevi (Phillip Morris Co.); D. Wu (Dartmouth College) 


\section{Micrometer-Scale Reliability: Dynamic Imaging of Magnetic Domain Walls}

\begin{abstract}
The purpose is the development of high-sensitivity, low-noise magnetic sensors for use as metrological tools in health care, homeland security and general information technology. In health care, non-invasive sensors are needed for medical evaluation in the areas of magneto-cardiography and magnetic bead tracking of blood flow and stem cells. In homeland security, sensors are needed to detect low levels of pathogens. In IT, sensors are needed for ND failure analysis and magnetic data storage, among others.
\end{abstract}

\section{Roy H. Geiss}

$\mathrm{T}$ he goal is to develop multi-level magnetic sensors with a sensitivity better than $1 \mathrm{p}$-tesla $/ \sqrt{ } \mathrm{Hz}$ at $1 \mathrm{~Hz}$ noise level that operate at room temperature and can be easily integrated with standard silicon electronics. This involves a many-faceted program that includes materials development, sensor fabrication, sensor characterization, modeling and nanomagnetodynamic imaging. The component being studied here concerns the dynamic observation of magnetic domain walls using Lorentz microscopy in the transmission electron microscope (TEM). Imaging of static magnetic domain walls in the TEM is not new, but our focus is to study the dynamics of domain wall-defect interactions and changes in spin distributions associated with defects or other discontinuities in nanodevices. The ultimate goal is to associate the dynamical interactions with electrical noise measurements in the same sensor.

The effort to image magnetic domain walls has just started. A $200 \mathrm{kV}$ TEM was obtained in 2003. A highresolution, high-speed CCD camera was installed, and highresolution, dynamic-magnetic imaging was successfully demonstrated. A sample holder with multiple current feed throughs was designed that allows the application of magnetic fields for in-situ testing. Thus far, we have obtained high-resolution images of domain walls at frame rates up to $15 \mathrm{~s}^{-1}$. In particular, we have filmed the formation and movement of Bloch lines and points in cross-tie walls in Ni80Fe20 films $50 \mathrm{~nm}$ thick, and have observed the pinning of cross-tie walls at small defects in a noncrystalline CoFeNiSiB alloy film provided by a U.S. magnetic sensor company. Both these dynamic experiments were performed by slowly varying the small local magnetic field in the neighborhood of the specimens in the TEM. Movement of domain walls was possible since the coercivity of both films was very small, on the order of a few oersteds.

The observation of pinning of the domain walls by small defects in the CoFeNiSiB film demonstrated
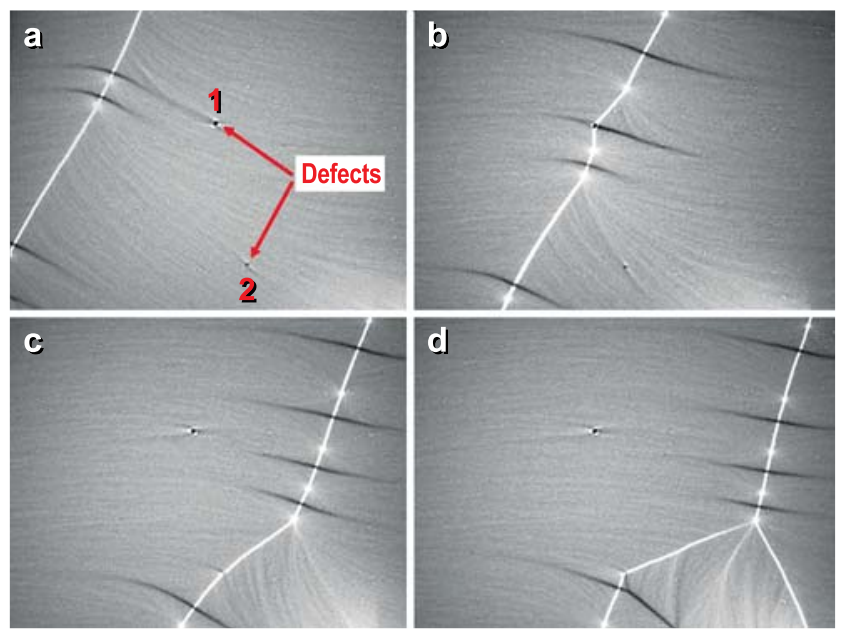

Figure 1: Images a to $\boldsymbol{d}$ are from a video sequence showing domain wall pinning. The cross-tie wall seen at the left-hand side in a slowly moves to the right under the application of a small magnetic field in $\boldsymbol{b}$ through $\boldsymbol{d}$. In $\boldsymbol{b}$ the wall is pinned by defect 1 . With continued applied field, the wall breaks free from defect 1 and moves to defect 2, where it is again pinned, as suggested in $c$ and definitely in $\boldsymbol{d}$. Defects 1 and 2 are $4 \mu \mathrm{m}$ apart.

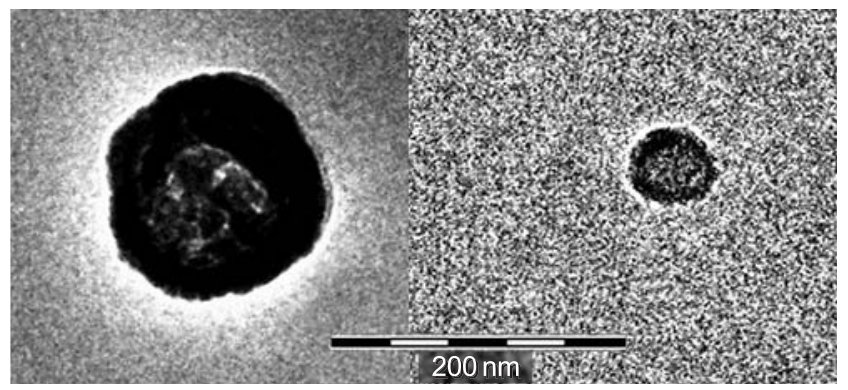

Figure 2: Images of the individual defects 1 and 2.

our capability to view interactions in real time. Further, with frame-by-frame analysis of the video, we determined which defects interacted with the domain walls. A few frames from a video sequence containing domain wall pinning are shown in Figure 1. High magnification images of the noncrystalline defects are given in Figure 2.

An abstract involving this work has been submitted to the "2004 Magnetism and Magnetic Materials" conference. Studies on smaller structures are the next phase.

\section{Contributors and Collaborators}

S. Russek, B. Rippard, D. Pappas (Magnetic Technology Division, NIST) 


\section{Nanotribology and Surface Properties}

\begin{abstract}
Accurate adhesion and friction measurements at the nanoscale are emerging as critical issues in device industries and nanotechnology. Measurements characterizing nanometer-thick molecular assemblies and surface textures to control surface properties and to ensure reliability and durability are also needed. Working with device and magnetic storage industries and other agencies, we have designed and built instruments to meet these needs. These instruments are housed in NIST's new Advanced Measurement Laboratory. The facility features vibration isolation, class 1000 clean room standards, and temperature control.
\end{abstract}

\section{Stephen M. Hsu}

$\mathrm{T}$ he nanotribology and surface properties project was initiated as a part of the MSEL Nanotechnology Initiative. The project aims to develop measurement capability for adhesion, friction, and surface forces at the nanoscale. We interact extensively with many industries and research centers in the U.S. and around the world to promote advances in measurement science and seek consensus standard harmonization in three areas: nanocontacts, nanolubrication, and surface texturing.

\section{Nanocontacts}

Adhesion and friction measurements at nanocontacts require accurate detection of normal and lateral forces at nanonewton levels. Scanning probe microscopes use sensitive cantilevers and laser diodes to detect these forces, but this approach introduces rotation of the tips and crosstalk among the xyz planes. To understand the influence of surface forces, many of which are functions of contact area, attention must be focused on determining the real area of contact.

To resolve some of the instrumental challenges, we have engaged instrument makers, such as Hysitron, Veeco, and others, to solicit their inputs and collaborations. At the same time, we have established our own capability in tip fabrication, cantilever spring constant calibration, and tip characterization. Three new instruments were successfully installed as a result of these collaborations: the NIST-Hysitron multiscale friction tester, the NIST nanoadhesion apparatus, and the first prototype of an interferometer microscope from Veeco. We are continuing to work with our partners to develop next-generation instruments as suggested by the Nanometrology Grand Challenges Workshop (NIST, January 2004).
The overall objective of the nanocontacts activity is to develop the constitutive equation of adhesion and friction including the various components of surface forces. With our new adhesion and nanofriction apparatus, we are quantifying the effects of plowing and electrostatic charge on measurements. We continue to work with our external academic partners (UC Berkeley, UC Davis, and Ohio State U) under the Nanotechnology Extramural Initiative to develop friction measurement via three approaches: friction measuring MEMS devices, AFM methods, and ultrahigh vacuum friction measurement. These efforts have been successful, and we have gained considerable understanding of how meniscus forces and electrostatic forces can exert significant effects on nanofriction measurements. A NIST special publication summarizing these findings is under preparation.

\section{Nanolubrication}

Molecular assemblies can be organized to impart hydrophobicity, anti-adhesion, and friction control characteristics on device surfaces. Supported by other agencies and the magnetic storage industry, this activity focuses on how to measure the effects of controlled composition and spacing on properties of nanometerthick films. An ultrahigh vacuum scanning tunneling microscope-atomic force microscope was installed in May 2004 to provide imaging capability at the molecular level. A micro-tribometer was also developed to measure the durability of these films. The synchrotron facility operated by the Division's Characterization Methods Group at Brookhaven National Laboratory continues to be vital in characterizing these complex molecular mixtures.

\section{Surface Texturing}

Surface texture increasingly is being considered as a tool to control surface energy, polarity, adhesion, and friction. Supported by other agencies and industries, we are pioneering the use of specific surface features such as dimples, triangles, and ellipses at microand nanoscale dimensions to supplement molecular assemblies to control surface properties of surfaces. An international cooperative study under the auspices of International Energy Agency (IEA) is underway.

\section{Contributors and Collaborators}

C. Ying, R. Gates, J. Chuang, J. Larson-Basse, L. Ives, D. Fischer (Ceramics Division, NIST); B. Bhushan (Ohio State U.); K. Komoupoulas (UC Berkeley); G. Liu (UC Davis); Y.T. Hsia (Seagate); J. Sengers (U. of Maryland); O. Warren (Hysitron); C. Su (Veeco) 


\section{Nanoscale Characterization by Electron Microscopy}

\begin{abstract}
Electron microscopy is used to characterize the structure and composition of materials at the nanometer scale to better understand and improve their properties. New measurement techniques in electron microscopy are being developed and applied to materials science research. The MSEL Electron Microscopy Facility primarily serves the Metallurgy, Ceramics, and Polymers Divisions as well as other NIST staff and outside collaborative research efforts.
\end{abstract}

\section{John E. Bonevich}

The MSEL Electron Microscopy Facility consists of two transmission electron microscopes, three scanning electron microscopes, a specimen preparation laboratory, and an image analysis/computational laboratory. The JEM3010 TEM can resolve atomic structures and employs an energy selecting imaging filter (IF) and X-ray detector (EDS) for analytical characterization of thin foil specimens. The S-4700-II FE-SEM employs electron backscattered diffraction/phase identification (EBSD) and EDS systems to characterize the crystallographic texture and composition of materials.

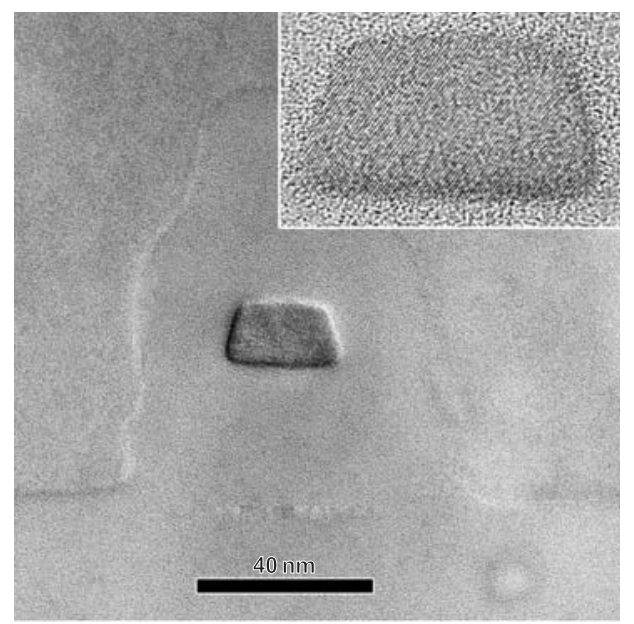

Figure 2: Confined Si single electron transistor device.

Highlights from the EM Facility for FY2004 include:

- A new, high-sensitivity EBSD CCD camera which acquires in excess of 70 patterns/second, was installed on the FE-SEM.

- 3D Chemical Imaging at the Nanoscale, a collaborative project with CSTL and PL on tomographic characterization of materials, was initiated (Figure 1).

- Research collaboration with the Semiconductor Electronics and Electricity Divisions (EEEL) has characterized quantum effects in confined Si devices (Figure 2).

- Sub-100 nm Ag interconnects formed by superconformal electrochemical deposition was characterized.

\section{Contributors and Collaborators}

D. Josell, T. Moffat, L. Bendersky (Metallurgy Division, NIST); I. Levin, B. Hockey (Ceramics Division, NIST); J.H.J. Scott (Surfaces \& Microanalysis Science Division, NIST); Z. Levine (Optoelectronics Division, NIST); E. Vogel (Semiconductor Electronics Division, NIST)
Figure 1: MgO cubes with surfaces decorated by small gold particles. These particle ensembles are used in model studies of $3 \mathrm{D}$ chemical imaging at the nanoscale (electron tomography). 


\section{Metrology for Nanoscale Properties: X-ray Methods}

\begin{abstract}
Macroscopic properties of technologically interesting materials originate from their underlying micro-structure. To design and understand improved materials, it is necessary to characterize the microstructure and correlate its changes to the macroscopic properties of interest. We especially focus on $x$-ray diffraction studies of biomedical, ferroelectric, optoelectronic, photovoltaic, semi-conducting, and other materials relevant to the health and microelectronics industries. In particular, studies of microstructural properties, such as strain and stress, crystalline defects, and texture, complement the information obtained by other techniques.
\end{abstract}

\section{Thomas A. Siewert and Davor Balzar}

$\mathrm{I}_{\mathrm{s}}^{\mathrm{n}}$ this report, we focus on two topics: the studies of superconducting tungsten thin films and comparison of the thin-film texture, as obtained by Electron Back Scatter Diffraction (EBSD) and x-ray diffraction (XRD). Tungsten superconducting transition-edge sensors (TES) are used in different astrophysics and astronomy applications, and as single-photon detectors for the quantum computing experiments. Such detectors comprise thin tungsten films and operate at temperatures close to absolute zero and to the critical superconducting transition temperature $(T c)$ of tungsten. Thin films, in general, contain both hightemperature $\alpha-\mathrm{W}$ phase (with $T c=15 \mathrm{mK}$ ) and $\beta-\mathrm{W}$ (A15 cubic structure with $T c=1-4 \mathrm{~K}$ ). Close to the $T c$, very small thermal changes, such as the absorption of a single photon, increase the film electrical resistance. The result is the production of an electrical output signal that corresponds directly to the detection of the absorbed photon energy. In general, residual stress can influence the $T c$ of the thin film both directly and by inducing the $\beta \rightarrow \alpha$ phase transformation. Thus, in order to control the $T c$, it is necessary to measure and control both the phase composition and residual stresses. Residual stresses in films depend on the preparation conditions, the thickness of the film, and the difference in thermal expansion coefficient between the film and the substrate. The total residual stress results from both the intrinsic (growth) stress and thermal stress. We measured the stress by XRD at both ambient and cryogenic temperatures. The sample was held in a continuous flow cryostat that was capable of achieving temperatures as low as $8 \mathrm{~K}$. The cryostat was mounted on a goniometer to enable the angle-dispersive XRD measurements. The shift of the $\alpha-\mathrm{W}\{110\}$ Bragg reflection was used to estimate stress at $8 \mathrm{~K}$, and directional measurement of the $\{321\}$ lattice spacings ( $\sin ^{2} \psi$ method) yielded the total residual stress at ambient temperature. The values obtained are about $0.7 \mathrm{GPa}$ and higher, where the bulk of the stress is due to the intrinsic (growth) component.

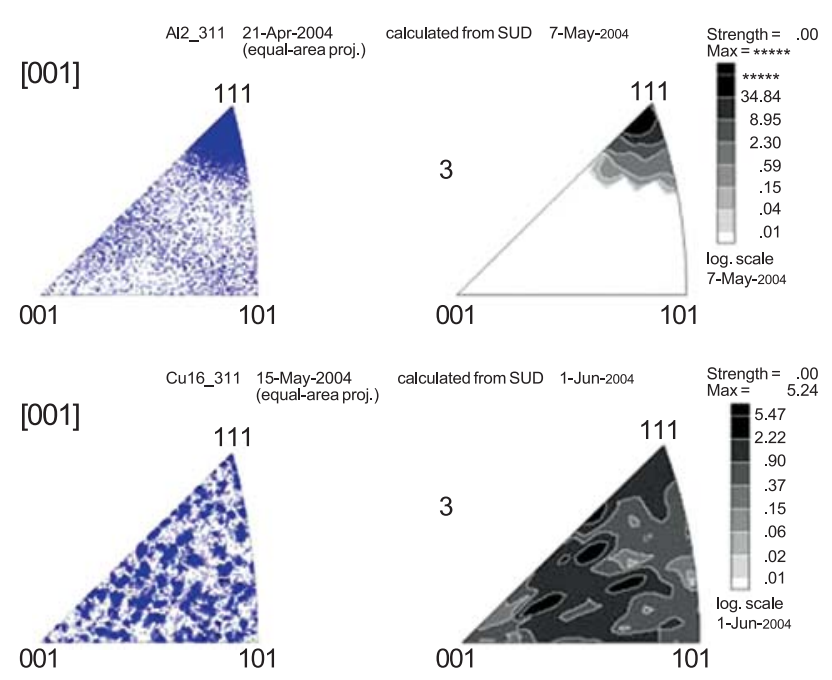

Figure 1: EBSD (left) and XRD (right) inverse pole figures of aluminum (top) and copper (bottom) thin films.

Texture has a large influence on many properties of thin films. XRD was the primary method for the characterization of texture for many years. Recently, alternative local techniques have been developed to bring the measurements to the nanoscale. This year, we compared measurements taken with conventional XRD and EBSD on two thin films: sputtered aluminum and electroplated copper. Both films had a thickness of about $1 \mu \mathrm{m}$ and were grown on [100] silicon wafers. The inverse pole figures for both samples and techniques are shown in Figure 1. It is obvious that the agreement between XRD and EBSD in case of aluminum film is very good, whereas it is rather poor for copper film. This is a consequence of different penetration depths of electrons and x-rays; while the aluminum sample is homogeneous through the film thickness, copper film is not. After sample preparation, electroplated copper is likely to undergo self-annealing at room temperature. This well-known phenomenon yields a difference in grain size and orientation between the surface and the bulk of the sample.

\section{Contributors and Collaborators}

A. Lita, J. Müller, R.H. Geiss, D.T. Read, M. Kopycinska-Müller, R. Keller (Materials Reliability Division, NIST); L. Kaatz, A. Chaudhuri (University of Denver) 


\section{Metrology for Nanoscale Properties: Brillouin Light Scattering}

\author{
Brillouin light scattering is being employed to \\ provide information on acoustic and magnetic \\ waves at gigahertz frequencies in thin-film \\ materials. Measurements and modeling in FY04 \\ have focused primarily on interactions of spin \\ waves in ferromagnetic films, elastic constants of \\ nanoporous low- $\kappa$ dielectrics, and propagation \\ of acoustic waves in nanopatterned polymers. \\ Ward Johnson, Sudook Kim, and
Colm Flannery
}

\section{Technical Description}

$\mathrm{B}^{\mathrm{t}}$ rillouin light scattering (BLS) is an experimental technique that measures the intensity of spectral components of light that is inelastically scattered by vibrational waves (phonons) or spin waves (magnons) in a material. Fabry-Perot interferometric techniques are used to acquire accumulated spectra through repeated mechanical sweeping of the etalon spacing.

BLS is the only laboratory technique that is currently available for detecting magnons of finite wavenumber. Because of this capability and because of innovations in interferometric techniques, BLS has been increasingly applied to problems in magnetics over the past couple of decades.

For characterization of phonons in thin films, BLS offers several advantages over other techniques. Since it detects phonons in the gigahertz range, elastic constants can be determined in films of submicron thickness without the complication of vibrational energy penetrating significantly into the substrate. Modes localized in submicron structures can be characterized. Spatial variations in elastic properties and vibrational patterns can be measured with a lateral resolution equal to the size of the laser focal spot on the specimen (typically, $\sim 50$ micrometers).

In this division, techniques are being developed for characterizing interactions of magnons in metallic ferromagnetic thin films. Such interactions limit the speed of magnetic-storage devices, spin-valve sensors, and other thin-film magnetic devices.

BLS measurements and modeling of vibrational modes are being pursued in several thin-film systems, including nanoporous low-dielectric-constant (low- $\kappa$ ) dielectrics, nanopatterned polymers, and arrays of molecular rotors.

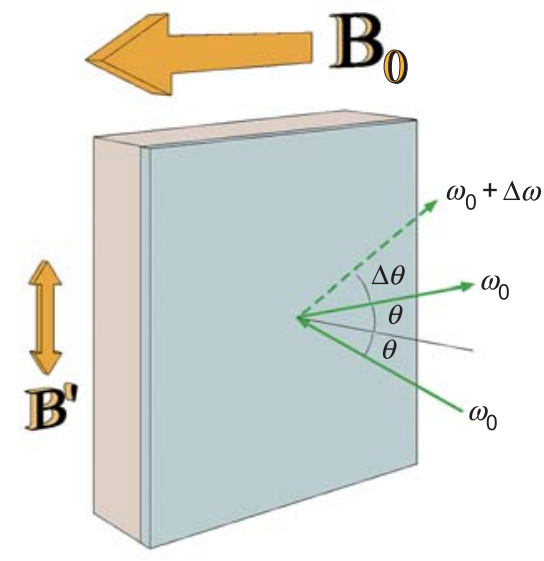

Figure 1: Scattering of light off waves in a thin film.

\section{Accomplishments}

During FY04, we developed and applied methods for measuring magnons that arise directly or indirectly from microwave pumping of metallic ferromagnetic films in a static magnetic field (Figure 1). BLS spectra were studied as a function of scattering angle, microwave power, and laser power to provide information on magnon-magnon interactions in $\mathrm{Ni}_{81} \mathrm{Fe}_{19}$. The results demonstrate, for the first time, detection of nonzero-wavevector magnons arising from the decay of pumped uniform-precession magnons in metallic thin films.

Young's modulus and Poisson's ratio in nanoporous low- $\kappa$ dielectric films were measured using conventional backscattering techniques. This research is described elsewhere in this annual report.

Measurements and modeling of vibrational modes in nanoimprinted polymers were pursued in collaboration with the University of Akron and the NIST Polymers Division. In addition to bulk and surface modes, low-frequency modes were detected and found to be consistent with vibrational localization in the imprinted lines. This result suggests the possibility of characterizing elastic properties on a scale of tens of nanometers.

\section{Contributors and Collaborators}

P. Kabos (Radio-Frequency Technology Division, NIST); S. Russek (Magnetic Technology Division, NIST); A. Slavin (Oakland Univ.); J. Michl (Univ. of Colorado, Chemistry Dept.); R. Horansky (Univ. of Colorado, Physics Dept.); C. Soles (Polymers Division, NIST); R.H. Hartschuh, A. Kisliuk, A. Sokolov (Univ. of Akron) 


\section{Nanostructure Fabrication Processes: Thin Film Stress Measurements}

The microelectronics community uses electrodeposition to produce solderable surface finishes, magnetic recording media, and copper interconnections in printed circuit boards and integrated circuits. These films tend to develop sizable mechanical stresses that can lead to loss of adhesion and the generation of bulk and surface defects. This project focuses on the measurement of these stresses which should enable the development of effective mitigation strategies.

\section{Gery R. Stafford and Ole Kongstein}

$\mathrm{E}$ lectrodeposited films tend to develop sizable mechanical stresses during deposition due to the nucleation and growth process or solution additives and alloying elements. We have established an optical bench dedicated to the in situ measurement of growth and residual stress during electrodeposition using the wafer curvature method. In one approach, a substrate of borosilicate glass is evaporated with $250 \mathrm{~nm}$ of gold. The force exerted on the cantilever by the electrodeposit causes curvature of the cantilever, which is monitored during electrodeposition. Forces on the order of $0.03 \mathrm{~N} / \mathrm{m}$ can be resolved during film deposition.

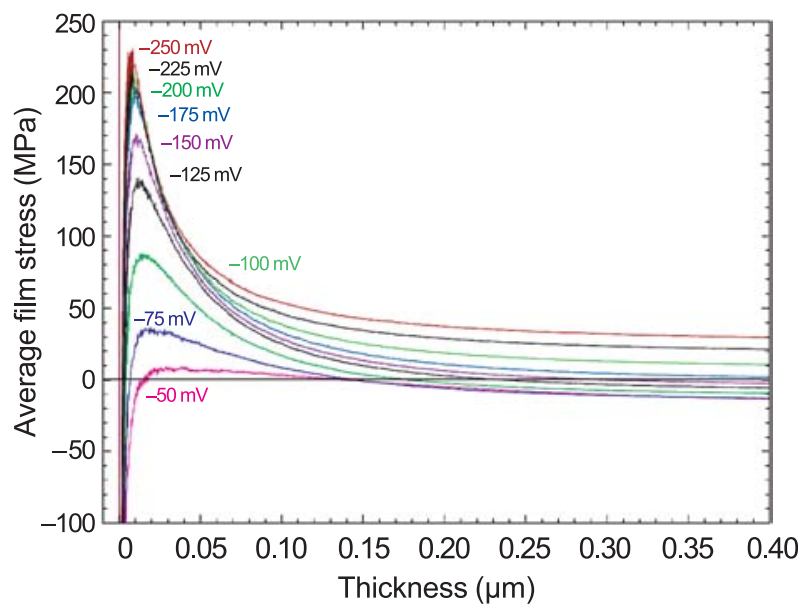

Figure 1: Average film stress during copper deposition.

Figure 1 shows the average in-plane stress associated with the deposition of copper from an additive-free sulfate electrolyte. The rapid rise in tensile stress (within the first $20 \mathrm{~nm}$ ) and its dependence on deposition potential are consistent with nuclei coalescence and grain boundary formation. The highest tensile stresses are associated with high nucleation densities which are obtained at more negative deposition potentials. As the deposit thickens, the average film stress decreases and becomes compressive in deposits formed at small deposition overpotentials. In the physical vapor deposition literature, this compressive stress has been attributed to the non-equilibrium concentration of mobile ad-atoms on the surface that are driven into the grain boundaries.

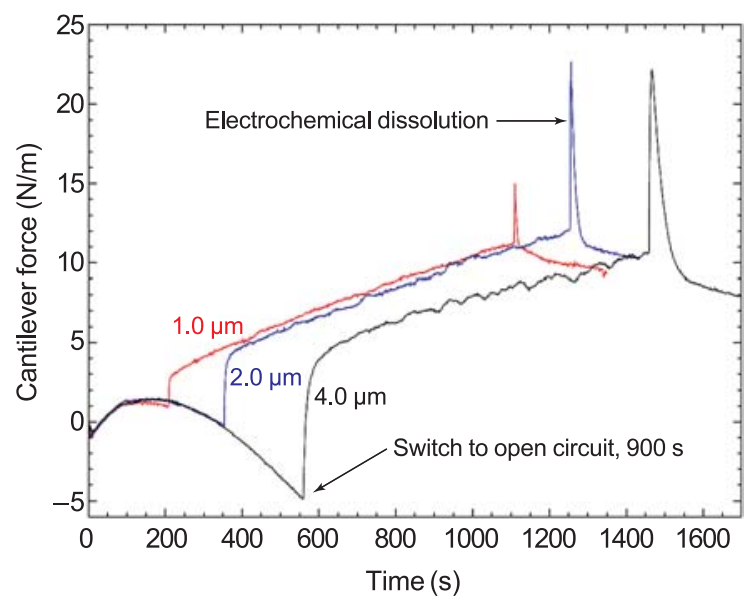

Figure 2: Force/width exerted on the cantilever during the electrochemical processing of $\mathrm{Sn}$ on $\mathrm{Cu}$.

We have also examined the force exerted onto a copper cantilever electrode during the deposition of matte tin (Sn), Figure 2. This is a particularly important system since the growth of tin whiskers, known to produce electrical short circuits and device failure, has been attributed to both the residual stress in the electrodeposit as well as that generated from intermetallic formation at the $\mathrm{Sn}-\mathrm{Cu}$ interface. The force curves show the following features: a tensile to compressive transition during deposition, a significant tensile relaxation when plating is discontinued, the development of tensile stress while the deposit is at open circuit, and a residual tensile stress after the deposit is electrochemically dissolved. The two latter features are attributed to the formation of the $\mathrm{Cu}_{6} \mathrm{Sn}_{5}$ intermetallic (IMC) at the $\mathrm{Sn}-\mathrm{Cu}$ interface. A tensile stress is generated since the IMC has approximately $6 \%$ smaller volume than a rule of mixture combination of the $\mathrm{Sn}$ and $\mathrm{Cu}$ reactants. Calculating the IMC thickness from the difference in $\mathrm{Sn}$ deposited and stripped, a nominal stress in the intermetallic was estimated to be $1.2 \mathrm{GPa}$ after only one hour. Future work will focus on determining to what degree this increases the compressive stress in the Sn electrodeposit.

\section{Contributors and Collaborators}

W. Boettinger, U. Bertocci (Metallurgy Division, NIST) 


\section{Nanomechanics: Coupling Modeling with Experiments}

\begin{abstract}
Knowledge of mechanical behavior is critical when designing for device performance and reliability, even for "non-mechanical" systems. However, nanoscale mechanical behavior (including failure) is inherently difficult to measure accurately, and existing modeling tools are only qualitative at best. We are developing modeling techniques that provide quantitative predictions and are validating these results experimentally.
\end{abstract}

\section{Lyle E. Levine and Anne M. Chaka (838)}

$\mathrm{M}$ echanics at the nanoscale is inherently difficult to model accurately. Finite element modeling (FEM) can effectively capture the elastic behavior of macroscopic structures but includes no accurate failure criteria since this depends upon atomic-scale behavior. Classical atomistic simulations can handle enough atoms (millions to billions) to model such events, but these potentials become inaccurate for large strains and they cannot effectively handle chemistry. Quantum-mechanicsbased simulations using density functional theory (DFT) are extremely accurate and handle the chemistry exactly, but such simulations are so CPU intensive that they can handle only a few hundred atoms. A combination of all three modeling techniques is required to accurately model device behavior at the nanoscale.

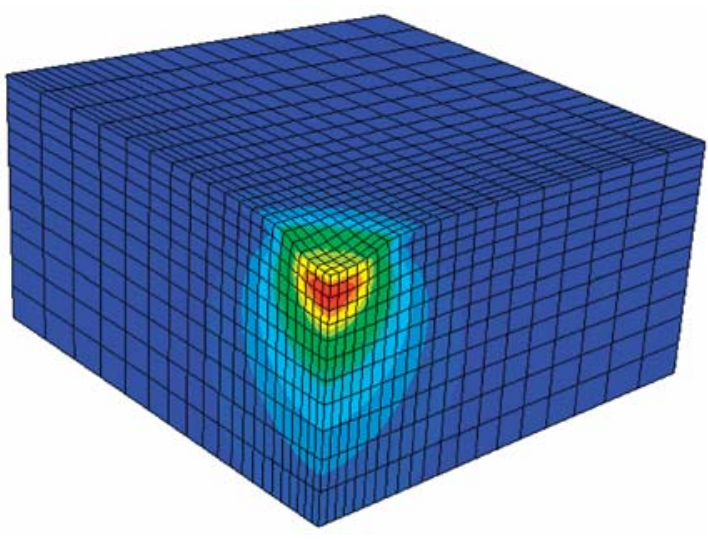

Figure 1: FEM model of a rigid $100 \mathrm{~nm}$ diameter sphere indenting an Al sample to a depth of $10 \mathrm{~nm}$.

Over the past year, we have developed techniques to handle such multiscale modeling for quasistatic applications. At the macroscale, FEM is used to simulate the elastic behavior of a nanomechanical system. Figure 1 shows an example in which an $\mathrm{Al}$ sample is being indented by a rigid $100 \mathrm{~nm}$ diameter sphere. The indenter and three of the sample quadrants have been removed to highlight the resulting Von Mises stress distribution after indenting $10 \mathrm{~nm}$. The FEM mesh is fine enough to use the predicted elastic displacement fields to generate boundary conditions and initial atom positions for an atomistic simulation using classical potentials.

The use of classical potentials in a large simulation cell allows the correct propagation of the long-range stresses to the critical regions where bond distortions are large or where chemistry effects need to be explored. In these critical regions, the techniques embed a DFT simulation. Iteratively, the critical region is relaxed using DFT, and the classical cell is relaxed using a Monte-Carlo algorithm. The first application of this hybrid technique was to determine the vacancy formation energy in aluminum as a function of distance (at a fixed angle) away from an edge dislocation. The simulation geometry is shown in Figure 2. The boundary conditions and initial atomic positions were calculated from the known elastic displacement field of an edge dislocation.

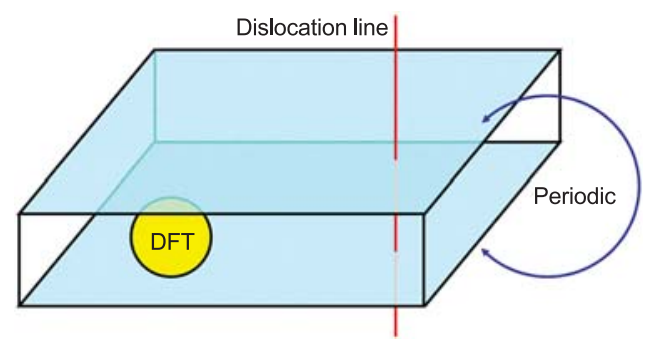

Figure 2: Diagram of a hybrid simulation for obtaining the vacancy formation energy at different positions relative to an edge dislocation. The large box represents the EAM cell in which the DFT region containing the vacancy was embedded.

After an initial dislocation nucleation event, nanoindentation progresses through the complex evolution of dislocation structures. For example, the raised lip around the indent is produced by large numbers of dislocations exiting the surface. We are working on modeling the early stages of this process using 3D dislocation dynamics and assuming a random distribution of dislocation sources in the sample.

Finally, connection to experimental measurements requires careful force calibration of the indenter and calibrated atomic force microscopy measurements (AFM) of the indenter tip. These calibrations are mostly complete, and the AFM data will be used to generate a FEM mesh for the simulations. Bulk single-crystal copper samples are being cut and polished using non-contact chemical methods to minimize the dislocation density.

\section{Contributors and Collaborators}

S.M. Khan, G. Levi, L. Ma, F. Tavazza (Metallurgy Division, NIST); B. Hockey, R. Machado, D. Smith, R. Wagner, D. Xiang (Ceramics Division, NIST) 


\section{Physical Properties of Thin Films and Nanostructures: Green's-Function Methods}

\begin{abstract}
Precise knowledge of atomistic configuration and strains in nanomaterials is needed for their characterization and development of new devices. We have developed computationally efficient Green's-function methods for calculation of lattice distortion, elastic strains and displacements in a variety of material systems. Our work is useful for interpretation of data obtained by AFAM, nanoindentation, and $S A W$ experiments.
\end{abstract}

\section{Vinod K. Tewary and Bo Yang}

\section{Technical Description}

Green's function (GF) provides a computationally Uefficient tool for interpretation of data obtained by nanoindentation and atomic force acoustic microscopy (AFAM) which is useful for elastic characterization of nanomaterials. The dynamic GF is used for modeling propagation of surface acoustic waves (SAWs) in solids and their phonon properties. The GF accounts for interfaces and free surfaces in the solid and its elastic anisotropy, which play relatively important roles in the elastic response of nanomaterials as compared to ordinary solids. We use GF to calculate the elastic displacement fields, interaction energy between the embedded nanostructure and other defects and free surfaces. The displacement field, due to a force at the surface, is used for interpretation of AFAM and nanoindentation measurements. The interaction energy of the nanostructures is an important factor in the stability and growth of the nanostructure.

We have developed GF methods both at the discrete atomistic and macro continuum scales. For a lattice model containing $\mathrm{N}$ atoms, the GF is given by:

$$
\mathbf{G}=\boldsymbol{\Phi}^{-1}
$$

where $\mathbf{G}$ and $\boldsymbol{\Phi}$ are $3 \mathrm{~N} \times 3 \mathrm{~N}$ matrices of $\mathrm{GF}$ and force constants respectively. The matrix inversion is carried out by using the discrete Fourier transform for a perfect lattice or numerically by computer simulation for a model crystallite as in molecular dynamics.

In order to model an embedded nanostructure in a host solid, or a thin film on a substrate, we define an initial GF for the host and the final GF. The final GF is related to the initial GF through the Dyson equation.

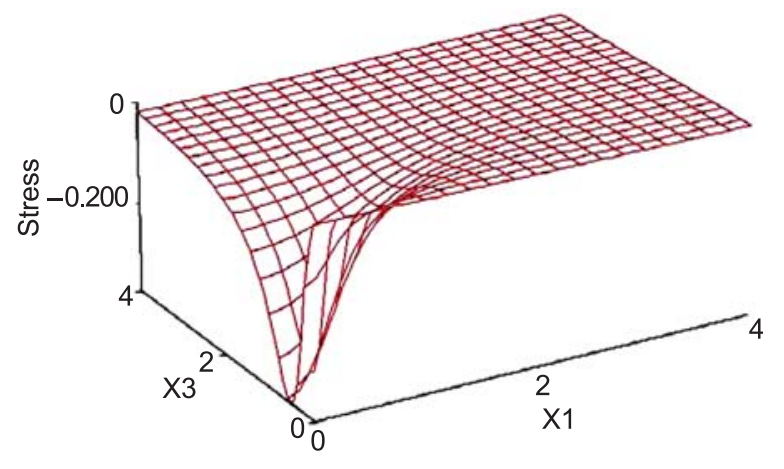

Figure 1: Stress distribution in Si due to a surface load.

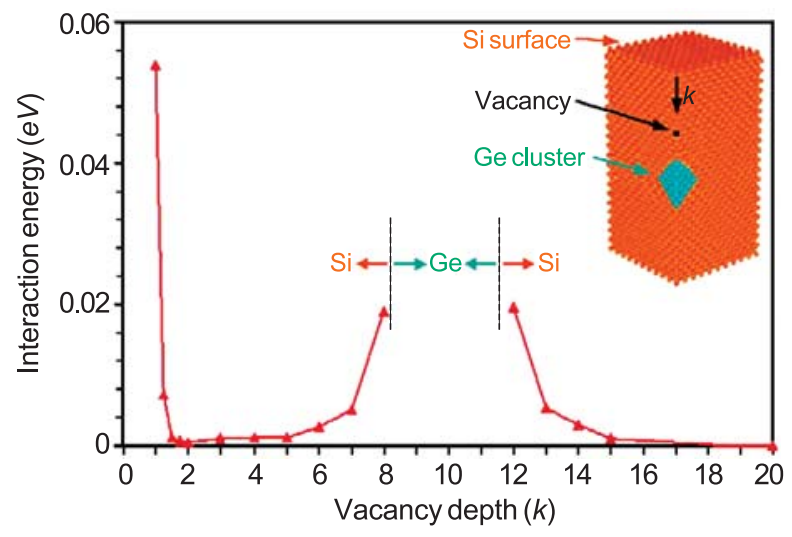

Figure 2: Interaction energy of a cluster of 147 Ge atoms with a vacancy and free surface in $\mathrm{Si}$.

In the continuum model, we simulate free surfaces and interfaces by applying the usual boundary conditions.

\section{Accomplishments}

We have carried out a detailed analysis of SAW measurements in a thin TiN film on $\mathrm{Si}$, calculated stresses in a wide range of solids, and modeled Ge clusters in Si containing a free surface and a vacancy. The work is reported in six papers. A library of GFs is available at www.ctcms.nist.gov/gf, which contains downloadable teaching and tutorial material on GFs and computer codes for calculation of GFs. A GF group of about 50 international users has been formed at CTCMS.

\section{Contributors and Collaborators}

D. Hurley, D.T. Read (Materials Reliability Division, NIST); L. Bartlolo (Kent State University); A. Powell (M.I.T.) 


\section{Micrometer-Scale Reliability: Bridging Length Scales}

\begin{abstract}
We have developed a multiscale model for nanostructures in solids. The model relates the physical processes at the interatomic level to measurable lattice distortions at the nanometer level and macroscopic stresses and strains. The model links the subnano (interatomic), nano (nanostructures), and macro length scales by integrating the powerful techniques of molecular dynamics, lattice-statics Green's functions, and continuum Green's functions.
\end{abstract}

\section{David T. Read and Vinod K. Tewary}

\section{Technical Description}

$\mathrm{M}$ athematical modeling is a very important tool for understanding the mechanical behavior of nanomaterials and for research and design of devices based upon nanostructures. A nanostructure needs to be modeled at the following scales: (i) the core region of the nanostructure (subnanometer), where the nonlinear effects may be significant; (ii) the region of the host solid around the nanostructure (nanometer); and (iii) free surfaces and interfaces in the host solid (macro). A nanostructure causes lattice distortion in the host solid that manifests as strain throughout the solid. The strain is essentially a continuum-model parameter, whereas the lattice distortions are discrete variables that must be calculated by use of a discrete lattice theory. Hence, one needs a multiscale model that relates the discrete lattice distortions at the microscopic scale to a measurable macroscopic parameter such as strain.

Conventional models of nanostructures are based upon either the continuum theory, which is not valid close to the defect, or molecular dynamics (MD) which is CPU intensive and usually limited to small crystallites, which may introduce spurious size effects. We need a computationally efficient multiscale model that links the length scales from subnano to macro and that can be used on an ordinary desktop. Such a model will be a valuable tool for research and engineering designs.

Our model is based upon the lattice-statics Green's function (LSGF) $\mathbf{G}$ that reduces asymptotically to the continuum Green's function (CGF). The displacement field in this model containing $\mathrm{N}$ atoms is given by:

$$
\mathbf{u}(\mathbf{l})=(1 / \mathrm{N}) \Sigma_{\mathbf{k}} \mathbf{G}(\mathbf{k}) \mathbf{F}(\mathbf{k}) \exp (\text { ik.l }),
$$

where $\mathbf{l}$ is a lattice site, $\mathbf{k}$ is a reciprocal space vector, and $\mathbf{F}(\mathbf{k})$ is the Kanzaki force, which is calculated by using MD without making the linear approximation.
For small k, $\mathbf{G}(\mathbf{k})$ reduces to CGF. Thus, for large 1, the equation reduces to macroscopic continuum theory while the discrete lattice effects are retained in $\mathbf{F}(\mathbf{k})$. Thus our model is truly multiscale since it seamlessly links the discrete atomistic effects in $\mathbf{F}(\mathbf{k})$ to macroscopic scales through the GF. Even for a million atom model, the calculation of GF takes only a few CPU seconds on a standard $3 \mathrm{GHz}$ desktop.

\section{Accomplishments}

Initial conference presentations and a journal paper on this new approach, first reported at the end of FY 2003 and during FY 2004, have been well received.

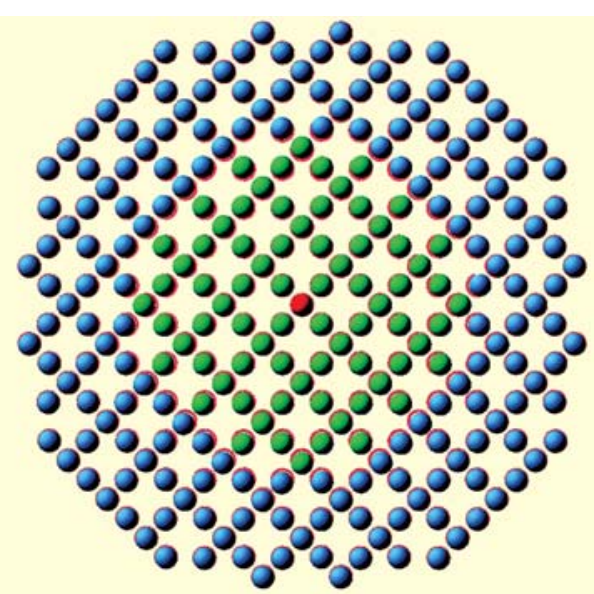

Figure 1: Section through Ge quantum dot in Si matrix after partial relaxation. The red circles indicate the original positions of the Si lattice sites; the atom in red is the center of the $Q D$.

This year, we set out to apply the approach to a more timely, but also more challenging, physical system, namely, a germanium "quantum dot" (QD) in a silicon matrix. On the GF side, the challenge was to treat the more complex diamond-cubic lattice structure; on the MD side, the more complex modified embedded atom model potential, needed for silicon and germanium, had to be coded and tested. As of this writing, the first iteration of the Si-Ge quantum dot is in progress. The figure shows the initial distortions of the diamond cubic lattice around a QD with $281 \mathrm{Ge}$ atoms; the method is capable of handling much larger QD, even with a desktop computer.

\section{Contributors and Collaborators}

R.R. Keller, B. Yang (Materials Reliability Division, NIST); E. Pan (Akron University) 


\section{Micrometer-Scale Reliability: Molecular Dynamics}

\begin{abstract}
It is widely anticipated that applications of nanomaterials will enable major advances in technology in a variety of fields, including sensors, high-strength materials, medicine, and others. Computer simulations seem to offer a path to quantitative understanding of the behavior of nanoscale materials. The form and parameters of the interatomic forces may turn out to be the most concise and useful representation of materials measurement results. Atomistic simulations are widely used in interpreting nanoscale phenomena at the boundary between mechanics and chemistry and to support the plausibility of proposed devices. We are developing the capability to use molecular-dynamics simulations to interpret our own measurements, and to assess the accuracy of proposed interatomic potentials.
\end{abstract}

\section{David T. Read}

$\mathrm{V}$ arious approaches to simulating atomic interactions have been reported. The first-principles approach uses quantum mechanical models of nuclei and electrons. Even though this approach typically includes only the valence electrons, it can handle only a few tens of atoms. Recently, in an innovative study, the first principles approach was used to search for new superalloys.

The molecular-mechanics approach, widely used for organic molecules such as proteins, requires explicit assumptions about atomic bonding, specifically, a list of which atoms are bonded to which other atoms. The bonds are represented by force laws, and the configuration of complex molecules can be studied. Different force laws may be used for certain types of atoms, depending on the chemical environment. For instance, the carbon-carbon force law in diamond may be different from that in graphite, because of the difference in local chemistry.

Molecular dynamics (MD) treats atoms or molecules as particles that follow Newton's laws of mechanics. The particles interact with a prescribed force law, which depends only on the chemical identity of the interacting atoms. The force laws are derived empirically, and the parameters are selected by fitting to measured properties, such as the elastic constants, the vacancy energy, the energy of sublimation, the phonon frequencies, and others. This approach can treat solids, liquids, or gases, and can model melting temperatures and equilibrium crystal structures.

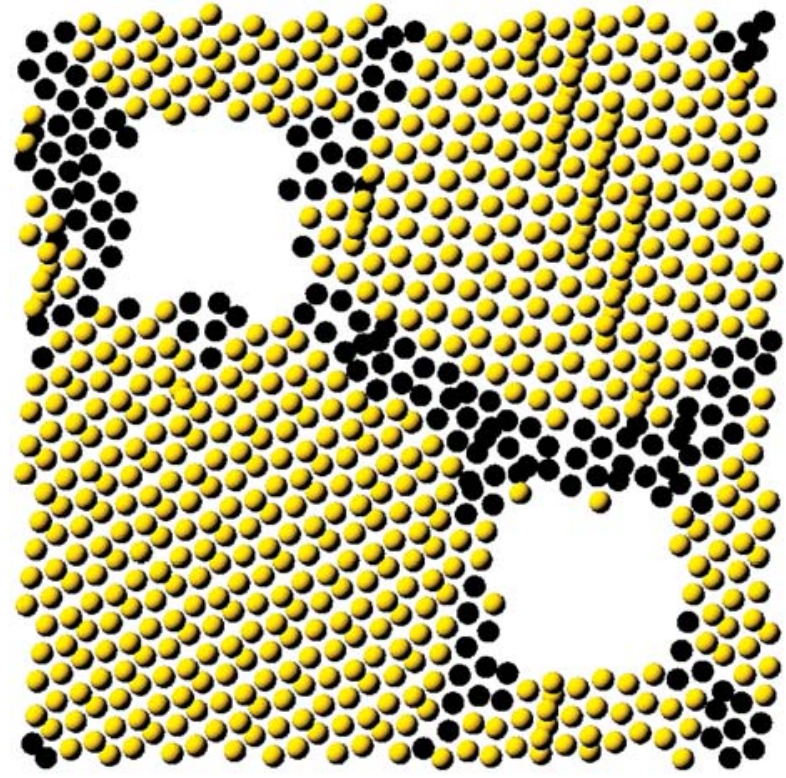

Figure 1: Cross-section from the center of an array of spheres (AS) model. Atoms shown in color have local fcc symmetry. Atoms shown as black have a lower local symmetry. Because of the periodic boundary conditions, this cell is effectively replicated indefinitely in all 3 dimensions.

Molecular-dynamics results obtained in the current fiscal year may indicate a mechanism for the recurring reports that the Young's (elastic) modulus values of thin films are lower than those of the corresponding (perfect) bulk material by $10 \%$ or more. Last year's report showed a high-resolution SEM micrograph of electrodeposited copper with a morphology of agglomerated spheres. Its Young's modulus was over $40 \%$ lower than the bulk value. This year, the elastic behavior of a simplified model of such a solid was simulated by molecular dynamics. Caution is in order. The size scale (number of atoms per sphere) and time scale of the simulation were much less than for the actual specimen. The results, averaged over a random set of crystal directions for the spheres, and for two sizes of spheres, produced a Young's modulus $35 \%$ below the bulk value. This was surprising because continuum-mechanics calculations of the modulus decrease produced by defects of like porosity show values proportional to the volume loss. The present MD results are much more dramatic because after the model was brought to equilibrium, it had a density of about $95 \%$ of the bulk value.

\section{Contributors and Collaborators}

J. Rifkin (University of Connecticut, author of MD program used) 


\section{Mechanical Metrology for Small-Scale Structures}

\begin{abstract}
Myriad industrial and biological systems are composed of small-scale structures for which the mechanical behavior is not accurately known. Optimizing the performance and reliability of these systems requires either mechanical property measurements on specimens of these structures harvested from the appropriate phases or interfaces of the system, or the ability to test these structures in situ. We are developing standardized testing configurations and methodologies for localized measurements of strength and fracture toughness of materials and interfaces at the micro- to nanometer-length scale.
\end{abstract}

\section{Edwin R. Fuller, Jr. and George D. Quinn}

$\mathrm{T}_{\mathrm{H}}^{\mathrm{h}}$ his project aims to: (1) measure mechanical properties of microstructures for myriad industrial and biological systems that cannot be fabricated in bulk samples; (2) study small-scale phenomena that may be controlled by surface effects, e.g., the influence of surface stresses on crack nucleation and extension; and (3) obtain quantitative mechanical property data of materials and interfaces for designing small-scale structures and components and for assessing their mechanical reliability. To address these goals, well-characterized testing configurations must be developed for small-scale measurements of strength and crack extension. We are pursuing four tasks: (1) configuration design and finite element analysis; (2) specimen fabrication; (3) mechanical testing and fracture analysis (fractography); and (4) length and force metrology. Work in the Ceramics Division this year has focused on the first and third areas. Two collaborations were established in the fabrication task: one with James A. Beall of the Quantum Electrical Metrology Division (817) in NIST Boulder, and one with Northwestern University. Work in the fourth task will

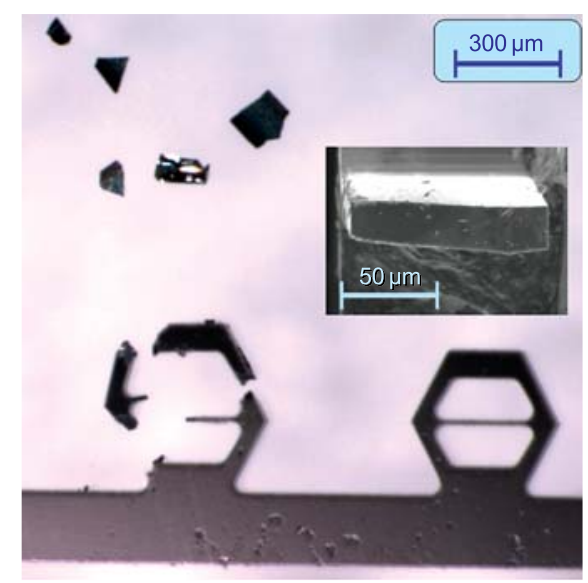

Figure 1: Prototype specimen design. come in subsequent years, most likely in collaboration with the Manufacturing Engineering Laboratory.

Significant progress has been made in the design of a compressively loaded test configuration with a welldefined, tensile gage section. Such a specimen can be loaded using a depth-sensing nanoindenter as a universal testing machine, thereby giving a record of both applied load and load-point displacement. One of these specimens, fabricated by James Beall from a silicon wafer by deep reactive ion etching, is shown in the right-side of Figure 1. The configuration is similar to a theta specimen, except that the geometry is hexagonal. When a load (per unit thickness) is applied to the top beam, a uniform uniaxial tensile stress results in the middle gage section. Finite element analysis gives (horizontal) gage section stresses on the order of $1.25 \mathrm{GPa}$ for $50 \mathrm{mN} / \mu \mathrm{m}$ of applied load. For a $2 \mathrm{~N}$ applied load, these $100 \mathrm{~mm}$-thick specimens generate $500 \mathrm{MPa}$ of tensile stress in the gage section. The left-side of Figure 1 shows a reconstructed failed specimen. The insert shows the fracture surface of the gage section, and the two [111] cleavage facets that were formed. Alternate geometries, including a round theta specimen, are also being considered.

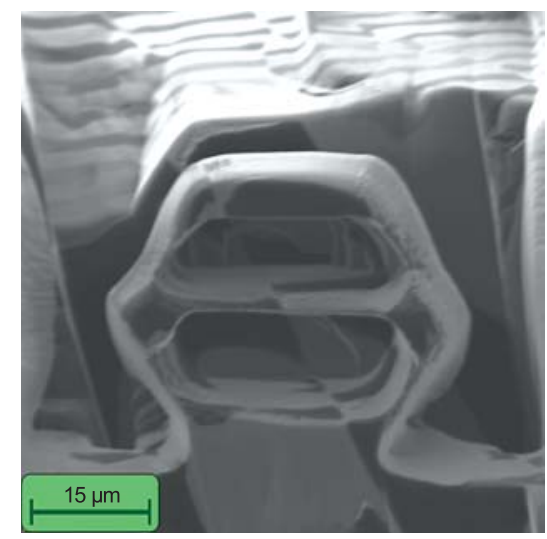

Figure 2: Hexagonal theta specimen by FIBing.

To extend this technique to a wide variety of materials and systems, general fabrication procedures need to be developed. Towards this objective, focusedion-beam (FIB) milling is being explored in collaboration with Northwestern University. Figure 2 shows our first attempt at producing a hexagonal theta specimen by FIBing. It has been scaled-down by about a factor of 10 , and is fabricated from a lamellar directionally solidified eutectic of $\mathrm{Ni}_{0.5} \mathrm{Co}_{0.5} \mathrm{O}$ and $\mathrm{ZrO}_{2}$.

\section{Contributors and Collaborators}

D. Xiang, D.T. Smith (Ceramics Division, NIST); A. Jillavenkatesa (Standards Services Division, NIST); J.A. Beall (Quantum Electrical Metrology Division, NIST); N. Alem, V.P. Dravid (Northwestern University) 


\section{Nanoindentation Methods and Standards}

\begin{abstract}
Nanoindentation has rapidly become the method of choice for quantitative determination of mechanical properties of thin films and small volumes of material, but standardization efforts have lagged far behind the application of the technique in industrial research and development. Over 1000 commercial nanoindentation instruments from a variety of manufacturers are currently in use, with no traceable force calibration and an extremely limited choice of standardized test methods. We work with standards groups and National Measurement Institutes around the world to develop standard reference materials (SRMs), traceable calibration transducers, and robust, reliable methods for obtaining and analyzing nanoindentation data, so that nanoindentation results can be used with confidence in product design and specification.
\end{abstract}

\section{Douglas T. Smith}

$\mathrm{N}$ anoindentation is the most commonly used method for determining hardness and elastic properties of small volumes of materials. In this technique, a diamond indenter is pushed into a specimen surface, and the force on and displacement into the surface are recorded. The technique is capable of providing information on the elastic and plastic deformation of a specimen for indentations as shallow as $5 \mathrm{~nm}$ to $10 \mathrm{~nm}$, and requires minimal specimen preparation effort. It is routinely used to measure the mechanical properties of thin films. However, there are only a very limited number of accepted test methods available, and no means to traceably calibrate or verify the performance of nanoindentation instruments. This leads to large interlaboratory variations in results, particularly for hard, high modulus materials, and prevents the use of nanoindentation in thin film or coating product specifications.

In many mechanical test methods, including nanoindentation, a force is applied to a specimen, and some displacement is measured. Traceable displacement measurement by interferometry is well established. Force measurement is more problematic, however, because the SI unit for force is still based on an artifact kilogram mass. The Microforce Competence Program at NIST has developed a primary realization of force, traceable to electronic and length SI units, for force calibration in the range $1 \mathrm{mN}$ to $10 \mathrm{nN}$. As part of this program, transfer force cells are being developed that will allow force calibration, traceable to NIST, for commercial nanomechanical test equipment such as nanoindentation machines and atomic force microscopes. One such transfer force cell was calibrated against the NIST primary force balance to an uncertainty less than $0.5 \%$ for forces in the range $0.05 \mathrm{mN}$ to $5.0 \mathrm{mN}$. That cell was then mounted, as if it were a specimen, in a popular commercial nanoindentation instrument that had been recently calibrated by its manufacturer using their recommended force calibration procedure. The figure shows the error in the nanoindentation force recorded by the instrument, relative to the traceable force cell reading. For forces above $2 \mathrm{mN}$, the recorded force is within the $1 \%$ uncertainty required by most draft standards for nanoindentation machines. However, for lower applied force, the error increases dramatically, and at $0.05 \mathrm{mN}$, the recorded force is almost $18 \%$ below the actual applied force.

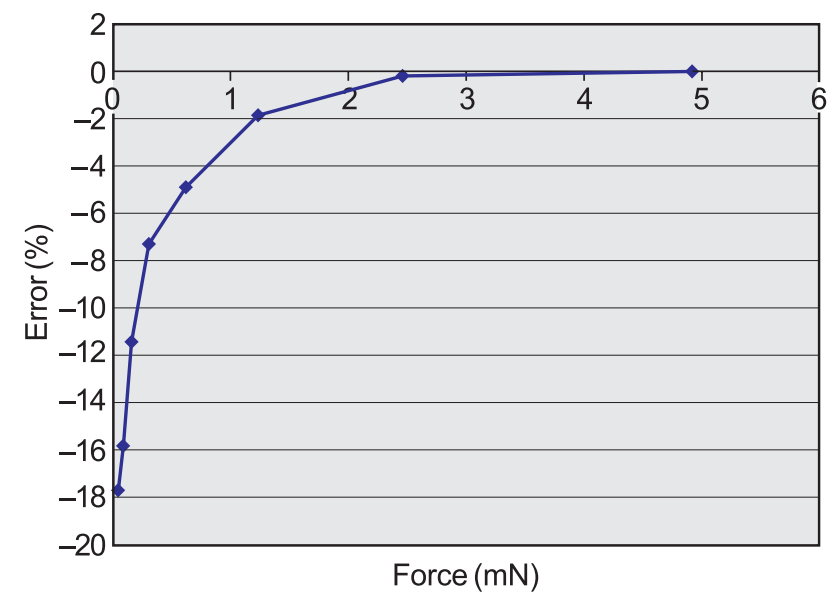

Figure 1: The error in the force applied to a specimen by a commercial nanoindentation instrument, as referenced to a traceably calibrated NIST force cell.

We are also working with both ASTM (E28.06.11) and ISO (TC 164/SC 3 ) on a wide range of standard test methods for nanoindentation, and an ISO document for the one method has now been approved (ISO 14577). In addition, the Ceramics and Materials Reliability Divisions at NIST are working with the Bundesanstalt für Materialforschung und-prüfung (BAM) in Germany to develop joint thin film SRMs (CRMs, or Certified Reference Materials, in Europe) for use in nanoindentation machine verification.

\section{Contributors and Collaborators}

D. Xiang, B. Hockey, G. Quinn (Ceramics Division, NIST); R. Machado (INMETRO, Brazil); J. Pratt (Manufacturing Engineering Laboratory, NIST); D. Hurley (Materials Reliability Division, NIST); U. Beck (BAM, Germany) 


\section{Nanostructure Fabrication Processes: Patterned Electrodeposition by Surfactant-Mediated Growth}

Novel structures and devices can be formed through template electrodeposition. The ultimate pattern resolution is determined by the size and packing of molecules that comprise the template and their ability to inhibit or catalyze various electrochemical reactions. In the past year, the effect of molecular functionality on the metal deposition process has been explored.

\section{Thomas P. Moffat and Michael J. Fasolka}

$\mathrm{O}$ ver the last decade, electrochemical processing has been undergoing a renaissance with the fabrication of new materials and novel microstructures. The development of new measurement techniques and metrological tools for studying controlled growth at fine length scales is the primary objective of this effort.

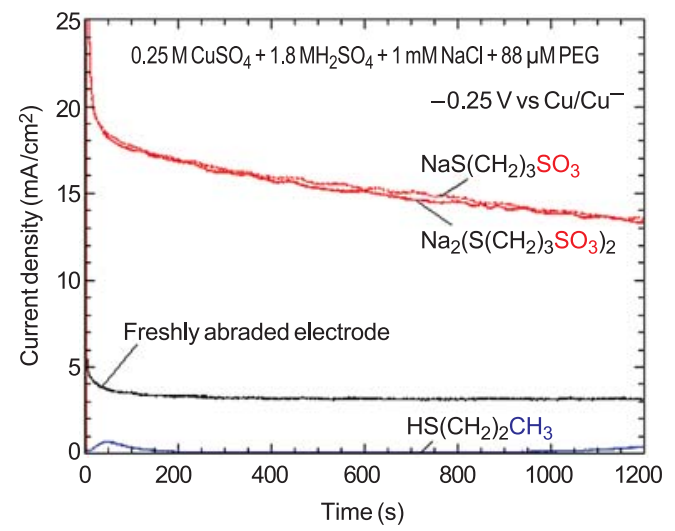

Figure 1: Influence of small changes in molecular structure, i.e., $\mathrm{SO}_{3}^{-}$vs. $\mathrm{CH}_{3}$, on the rate of copper deposition.

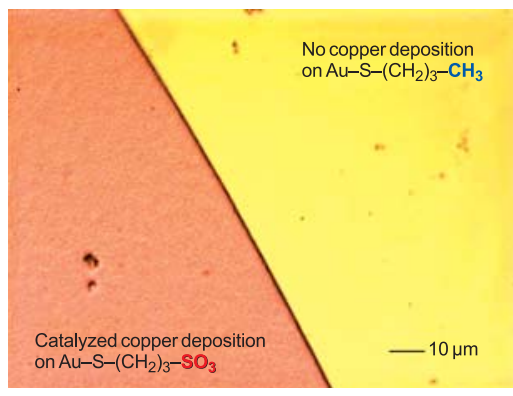

Figure 2: Copper deposition on an alknethiol derivatized gold substrate only occurs in regions bearing $-\mathrm{SO}_{3}^{-}$terminated molecules.

The three key metrology issues addressed are:

1. The rate differentiation accessible via surfactant mediated metal film growth;
2. The role of molecular functionality of the surfactant and robust design rules; and

3. The anisotropy induced in the electrocrystallization reaction rate for a given surfactant.

As an example, an alkanethiol monolayer film can either block or accelerate metal deposition depending on the molecular functionality of its terminal group. A dynamic range spanning several orders of magnitude is possible depending on the system in question (Figures 1 and 2). Based on these measurements, selective metal deposition may be obtained by patterning a substrate with the appropriate molecule.
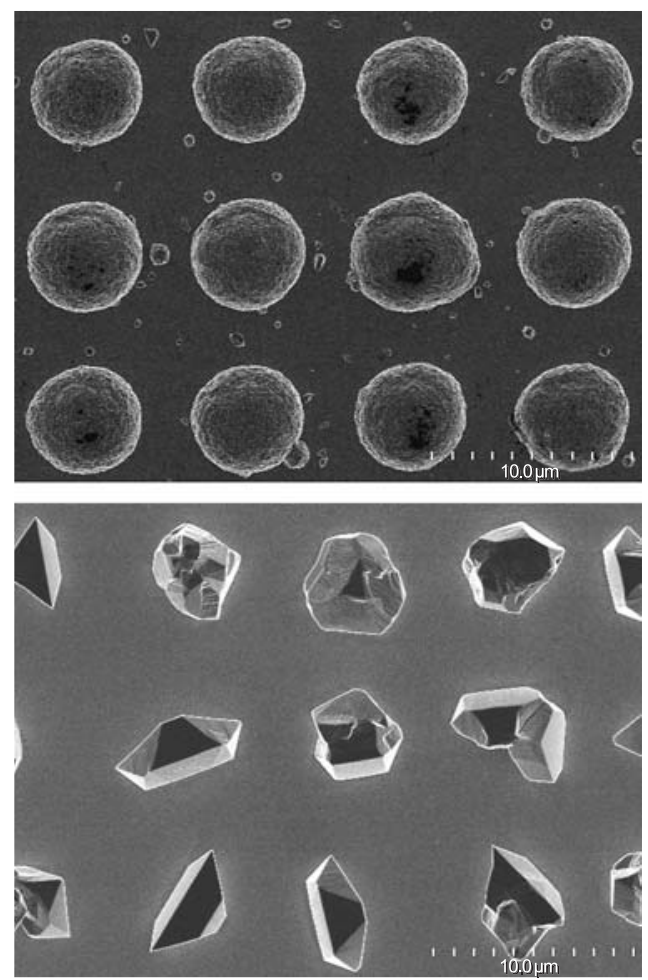

Figure 3: Spatially patterned monolayer films used to control nucleation and anisotropic growth of copper crystals.

Through contact printing, higher resolution patterning is possible, along with some interesting opportunities for high-throughput combinatorial research. By varying the surface chemistry, growth of isotropic or faceted crystals has been shown to be possible (Figure 3).

\section{Contributors and Collaborators}

D. Josell, J. Mallett, W.F. Egelhoff (Metallurgy Division, NIST); M. Walker, L. Richter (CSTL, NIST) 


\section{Chemistry and Structure of Nanomaterials}

Successful nanoscale materials fabrication is empowered by a detailed knowledge of the chemistry and structure of surface bound molecules; e.g., the optimization of self-assembled monolayers, molecular templates, micro-electro-mechanical system lubricants, and functionalized nanotubes. Near-Edge X-ray Absorption Fine Structure (NEXAFS) spectroscopy is ideally suited to measure non-destructively chemical bond concentration, rehybrization, and orientation with sub-monolayer molecular sensitivity in diverse nanoscale materials. Furthermore, NEXAFS can distinguish chemical bonding in the light elements, measure the orientation of interfacial molecules, and separately measure surface versus bulk chemistry simultaneously.

\section{Daniel A. Fischer}

$\mathrm{M}$ aterials having low energy surfaces are used in many applications, for example, in non-wetting surfaces or fouling resistant marine coatings. We have produced a photo-responsive polymer surface by combining the reversible photo-switching nature of azobenzene with the self-assembly nature and low surface energy properties of semi-fluorinated segments, to create a fluoroazobenzene molecule surface. Upon UV exposure, this surface reorients between hydrophobic and less hydrophobic states, as shown in Figure 1 (left upper and lower panels). For such surfaces, one could imagine applications ranging from low cost surface patterning to polymer surfaces that would adsorb biological macromolecules on cue.

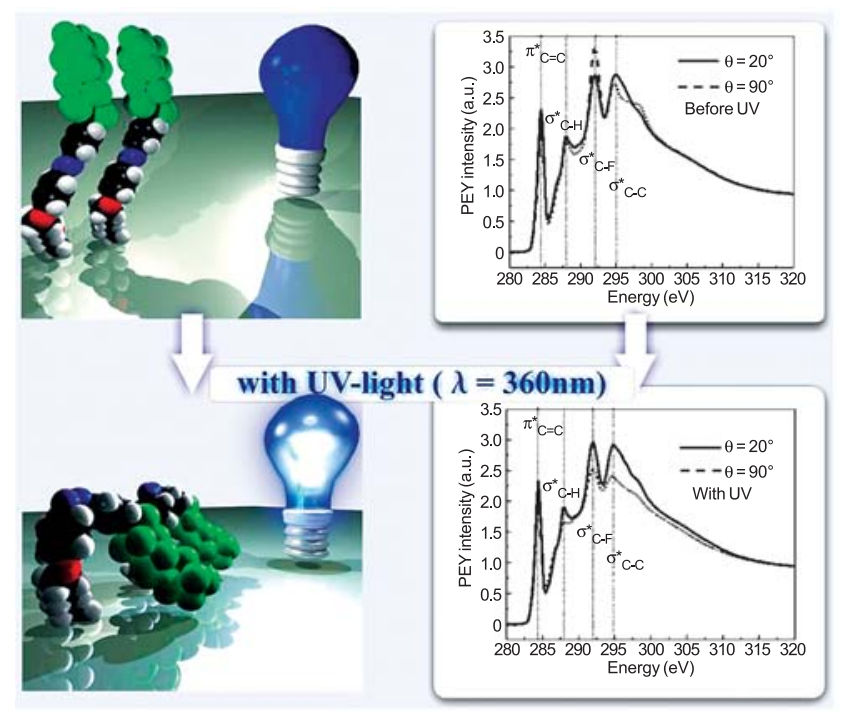

Figure 1: UV light reorients fluorobenzene semi-fluorinated segments (green) downwards, i.e., to a less hydrophobic state.
We have utilized NEXAFS to observe, verify, and quantify the reversible cis-trans molecular conformation transformation from hydrophobic to less hydrophobic states. The right panels of Figure 1 (upper and lower) show polarization dependent NEXAFS anisotropy behavior of the $\mathrm{C}-\mathrm{F}$ and $\mathrm{C}-\mathrm{C}$ peaks which reverse with in situ UV light exposure highlighting the reorientation of the semi-fluorinated segments.

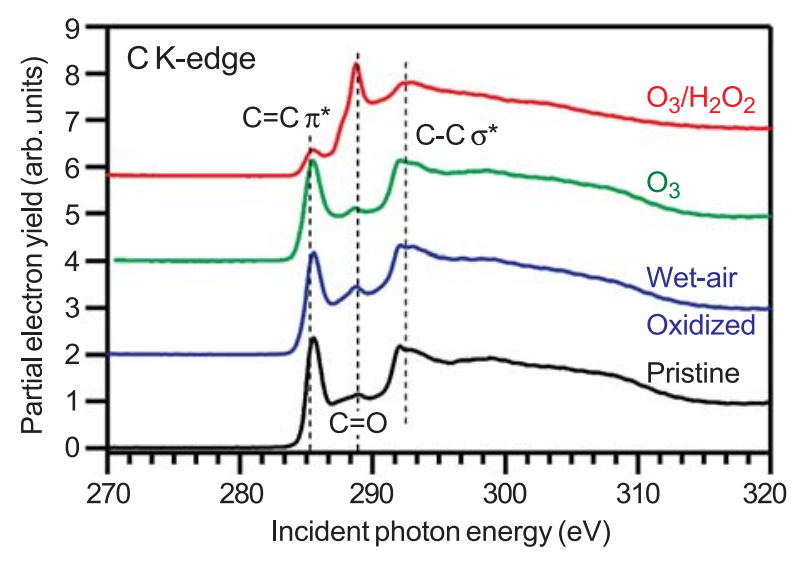

Figure 2: Carbon NEXAFS of oxidized/functionalized nanotubes.

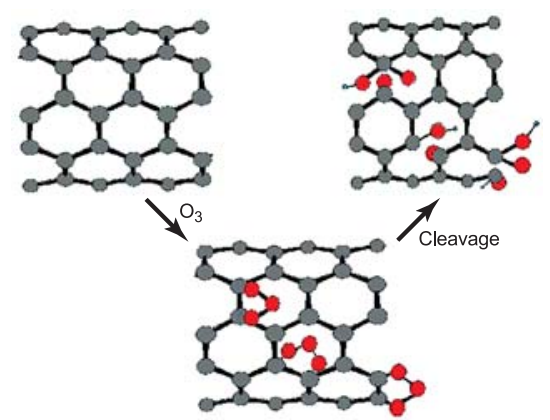

Figure 3: Model of peroxide functionalization of nanotubes.

Application of NEXAFS spectroscopy to the study of electronic structure and chemical composition is illustrated in Figure 2 for various chemically functionalized, singlewalled, carbon nanotubes. Upon peroxide functionalization, the $\mathrm{C}=\mathrm{C}$ ring resonance is greatly diminished on extensive sidewall functionalization indicating loss of extended conjugation and disruption of nanotube electronic structure. The $\mathrm{C}=\mathrm{O}$ peak intensity is greatest for peroxide chemistry. NEXAFS spectroscopy supports a model of peroxide funtionalization, shown in Figure 3.

\section{Contributors and Collaborators}

S. Samabasivan (Ceramics Division, NIST); X. Li, C.K. Ober (Cornell); A. Hexemer, E.J. Kramer (UCSB); S. Banerjee, T. Benny, S.S. Wong (SUSB); J.A. Misewich (BNL) 


\section{Thermochemistry and Metrology of Interfacial Interactions}

Reactions occurring at interfaces during the high-temperature processing of complex electronic materials involve diverse chemical compositions. To optimize the design and control of these interfaces, we are developing a generic thermodynamic/kinetic model for interfacial interactions. The model includes modification of bulk reaction and mass transport parameters to accommodate nanoscale observations.

\section{Lawrence P. Cook, Winnie Wong-Ng, and Igor Levin}

State-of-the-art electronic devices contain complex materials with chemical constituents ranging widely over the periodic table; furthermore, new materials are continually being added. Many electronic packaging materials consist of metals, ceramics, and semiconductors, with bi-phasic interfaces of several types. Processing temperatures may extend up to $900{ }^{\circ} \mathrm{C}$ where diffusion and reaction can be significant. Two principal problems arise in the treatment of such interactions: 1) the application of equilibrium thermodynamic data to the non-equilibrium growth of interfacial reaction zones, and 2) the transition from micro-scale (bulk dominated), to nanoscale (surface dominated) phenomena. To address these issues, we have instituted a combined thermodynamic/kinetic approach to investigate the model system $\mathrm{Ag}-\mathrm{Bi}_{2} \mathrm{O}_{3}-\mathrm{Nb}_{2} \mathrm{O}_{5}$-O, as outlined in Figure 1.

\section{$\mathrm{Ag}-\mathrm{Bi}_{2} \mathrm{O}_{3}-\mathrm{Nb}_{2} \mathrm{O}_{5}-\mathrm{O}$ Model System}

Thermodynamics

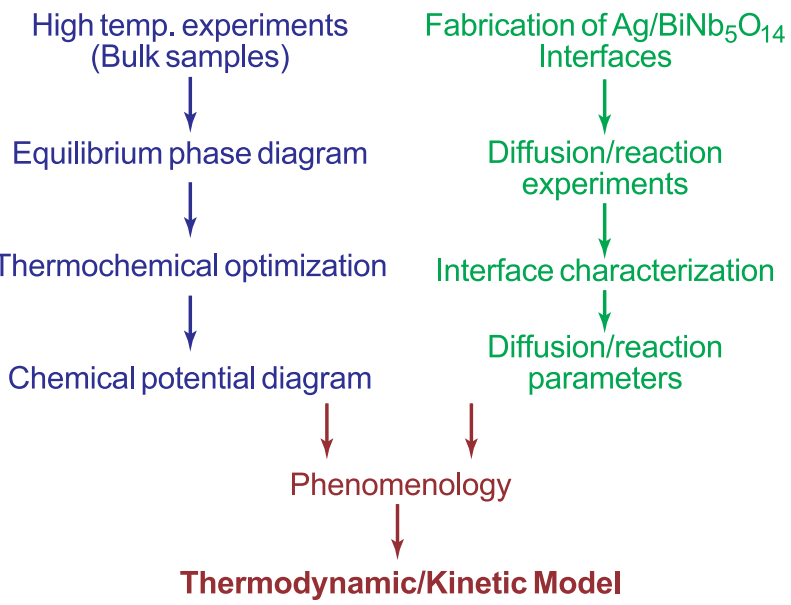

Figure 1: Flow chart for development of interfacial model based on $\mathrm{Ag}-\mathrm{Bi}_{2} \mathrm{O}_{3}-\mathrm{Nb}_{2} \mathrm{O}_{5}-\mathrm{O}$.

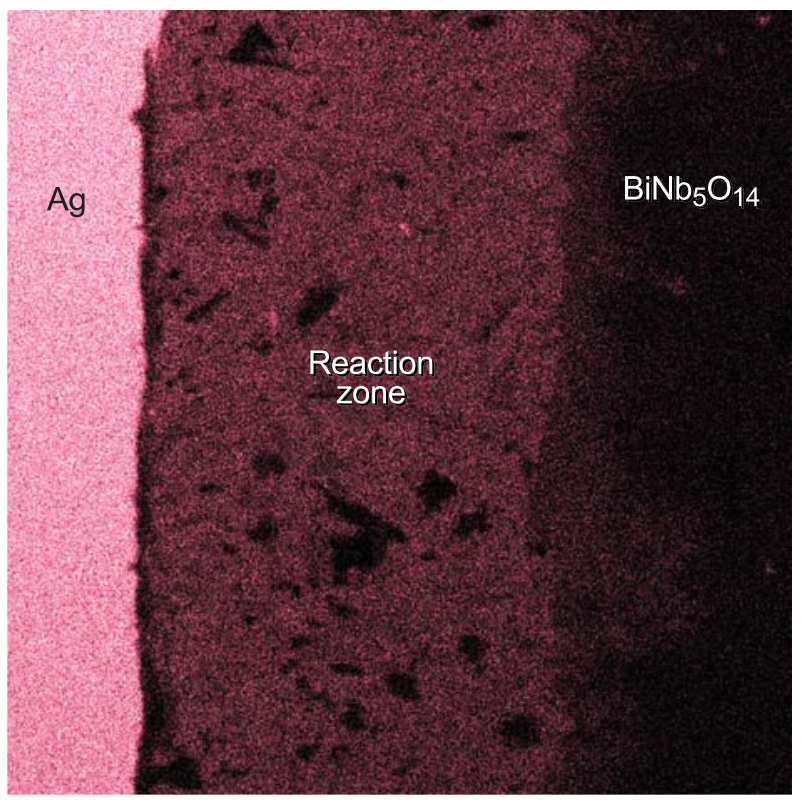

Figure 2: $\mathrm{Ag} \mathrm{L}_{\alpha} \mathrm{X}$-ray map of $\mathrm{Ag} / \mathrm{BiNb}_{5} \mathrm{O}_{14}$ reaction zone $\left(\approx 80 \mu \mathrm{m}\right.$ wide) produced by annealing in air for $10 \mathrm{~h}$ at $850{ }^{\circ} \mathrm{C}$, with an applied uniaxial compression of $\approx 1 \mathrm{MPa}$.

Progress includes determination of a preliminary phase diagram at $850^{\circ} \mathrm{C}$, and development of a provisional thermodynamic model. Subsequent construction of a chemical potential diagram has allowed us to predict alternative diffusion and reaction paths. Observations on $\mathrm{Ag} / \mathrm{BiNb}_{5} \mathrm{O}_{14}$ reaction couples (Figure 2) confirm the relatively high thermodynamic mobility of Ag. Thermogravimetric data on the interfacial reaction require more than a simple parabolic rate law, due to the rapid initial spread of Ag along reaction site surfaces. By comparison with particulate interfacial systems, a two-step reaction model is proposed, for which rate constants can be obtained.

Data for the model system are of direct interest to the electronics community because $\mathrm{Ag}$ is an important metallization component in many dielectric ceramics. It is anticipated that continued work on $\mathrm{Ag}-\mathrm{Bi}_{2} \mathrm{O}_{3}-\mathrm{Nb}_{2} \mathrm{O}_{5}-\mathrm{O}$ will lead to the goal of a comprehensive thermodynamic/kinetic model. The model will be tested, iteratively refined, and extended to other systems.

\section{Contributors and Collaborators}

M.D. Vaudin, P.K. Schenck, T. Vanderah, M. Green (Ceramics Division, NIST); W. Luecke (Metallurgy Division, NIST); C. Randall, M. Lanagan (Center for Dielectric Studies, Pennsylvania State University) 


\section{Phase Relations of High $\mathrm{T}_{\mathrm{c}}$ Superconductors}

Phase equilibria data are needed for quality control, cost reductions, performance enhancements, and optimal processing in high $T_{c}$ technology. These needs are being addressed through studies of the phase equilibria and kinetics of $\mathrm{Ba}_{2} \mathrm{YCu}_{3} \mathrm{O}_{6+x}$ formation from barium fluoride amorphous precursor films, determination of $\mathrm{Ba}_{2} \mathrm{RCu}_{3} \mathrm{O}_{6+x}$ $(R=D y, Y b)$ phase diagrams, and investigations of superconductor/buffer layer interactions: all play critical roles in the development of RABiTS/IBAD coated conductor technology.

\section{Winnie Wong-Ng, Lawrence P. Cook, and Igor Levin}

$\mathrm{P}$ hase diagrams serve as "blue prints" for successful processing of high $\mathrm{T}_{\mathrm{c}}$ superconductor materials. In the past year, as an integral part of the DOE intensive $R \& D$ program on high $T_{c}$ wire and cable applications, we have continued to provide critical data for the development of practical superconductors. Our research was related to two groups of superconductors: (1) $\mathrm{Ba}_{2} \mathrm{RCu}_{3} \mathrm{O}_{7}$ $(\mathrm{R}=\mathrm{Dy}, \mathrm{Yb})$ coated conductors produced by rolling assisted biaxially textured substrate/ion beam assisted deposition (RABiTS/IBAD); and (2) $\mathrm{MgB}_{2}$.

Our main effort has been focused on the determination of $\mathrm{BaO}-\mathrm{R}_{2} \mathrm{O}_{3}-\mathrm{CuO}_{\mathrm{x}}$ phase relations as a function of oxygen pressure, $\mathrm{p}_{\mathrm{O} 2}$, and choice of lanthanides. These studies were completed under carbonate-free conditions, to match better the processing conditions of RABiTS/IBAD conductors. This year we have completed the study of the two systems with $\mathrm{R}=\mathrm{Dy}$ and $\mathrm{Yb}$. A trend in phase formation and tie-line $\mathrm{Ba}_{2} \mathrm{RCu}_{3} \mathrm{O}_{7}$ relationships was observed in the present work which provides a general correlation for $\mathrm{R}=\mathrm{Nd}, \mathrm{Sm}, \mathrm{Eu}, \mathrm{Gd}, \mathrm{Dy}, \mathrm{Ho}, \mathrm{Y}, \mathrm{Er}$, and $\mathrm{Yb}$ with respect to the size of the lanthanides. In collaboration with Brookhaven National Laboratory and California Institute of Technology, an understanding of the effect of strain on the trend of the orthorhombic to tetragonal phase transition temperature of $\mathrm{Ba}_{2} \mathrm{RCu}_{3} \mathrm{O}_{7}$ was obtained through modeling. By mixing the smaller lanthanides $\mathrm{R}^{\prime}$ with the larger $\mathrm{R}$ in the $\mathrm{Ba}_{2-\mathrm{x}}\left(\mathrm{R}_{1+\mathrm{x}-\mathrm{y}} \mathrm{R}_{\mathrm{y}}\right) \mathrm{Cu}_{3} \mathrm{O}_{6+\mathrm{z}}$ superconductor, both flux-pinning and melting properties can be tailored and optimized. A trend in solid solution extent as related to the size of $\mathrm{R}$ was observed in $\mathrm{Ba}_{2-\mathrm{x}}\left(\mathrm{R}_{1+\mathrm{x}-\mathrm{y}} \mathrm{Y}_{\mathrm{y}}\right) \mathrm{Cu}_{3} \mathrm{O}_{\mathrm{z}}(\mathrm{R}=\mathrm{Sm}, \mathrm{Eu}$ and $\mathrm{Gd})$.

The " $\mathrm{BaF}_{2}$ ex-situ" process is a promising method for production of $\mathrm{Ba}_{2} \mathrm{YCu}_{3} \mathrm{O}_{7}$ superconducting wire and cable. Previously, using a controlled-atmosphere apparatus, we determined the presence of low-temperature liquids in the multicomponent reciprocal $\mathrm{Ba}-\mathrm{Y}-\mathrm{Cu} / / \mathrm{O}, \mathrm{F}$ system. This year, we identified YOF (Figure 1) as an

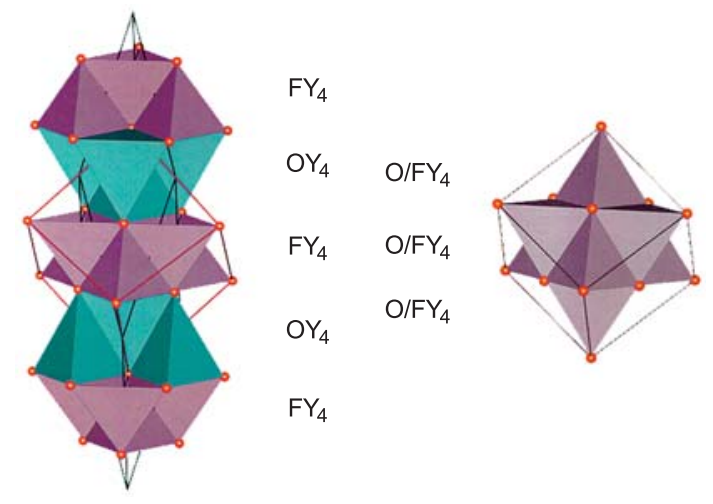

Figure 1: Schematic of the YOF rhombohedral structure (left, showing layers of $\left[\mathrm{OY}_{4}\right]$ and $\left[\mathrm{FY}_{4}\right]$ tetrahedra) and the cubic fluorite structure (right).

important intermediate phase in the system with a low temperature reversible order-disorder phase transition near the initial melting of fluoride-rich melts. These more detailed studies have allowed better definition of the region of low-temperature melting. Also, phase diagrams of the pertinent subsystems of $\mathrm{BaO}-\mathrm{Y}_{2} \mathrm{O}_{3}-\mathrm{CeO}_{2}-\mathrm{CuO}_{\mathrm{x}}$ representing the interaction of product $\mathrm{Ba}_{2} \mathrm{YCu}_{3} \mathrm{O}_{7}$ with the $\mathrm{CeO}_{2}$ buffer layer were determined.

To control film properties, it is important to understand the details of $\mathrm{Ba}_{2} \mathrm{YCu}_{3} \mathrm{O}_{7}$ formation from " $\mathrm{BaF}_{2}$ " films. High temperature $\mathrm{x}$-ray diffraction and transmission electron microscopy have been applied to compare the detailed conversion mechanism of films prepared with different precursors on $\mathrm{SrTiO}_{3}$ substrates and on RABiTS substrates (both provided by ORNL). A phase with a $\mathrm{Ba}\left(\mathrm{O}_{\mathrm{x}} \mathrm{F}_{\mathrm{y}}\right)$-based superlattice has been found to be involved as an intermediate product. Its presence may prove to be an important factor influencing the kinetics of $\mathrm{Ba}_{2} \mathrm{YCu}_{3} \mathrm{O}_{7}$ formation.

Studies on $\mathrm{MgB}_{2}$ also continued with measurements of the enthalpy of formation (by solution calorimetry) and vapor pressure (by thermogravimetric effusion). Attention was focused on the significant sources of variability in these properties. These data are essential for reproducible processing of $\mathrm{MgB}_{2}$ films, wire and cable.

\section{Contributors and Collaborators}

Q. Huang (NIST Center for Neutron Research); M. Vaudin, P. Schenck (Ceramics Division, NIST); R. Shull (Metallurgy Division, NIST); R. Klein (Biotechnology Division, NIST); R. Feenstra, A. Goyal (ORNL); T. Holesinger (LANL); M. Rupich (ASC); J. Kaduk (BP-Amoco); T. Haugan (U.S. Air Force); P. Canfield (Ames Lab); R. Meng (U. of Houston); D. Welch (BNL), H.B. Su (Caltech) 


\section{NIST Combinatorial Methods Center (NCMC) Pioneer and Partner in Accelerated Materials Research}

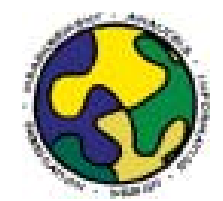

\begin{abstract}
Combinatorial and high-throughput (C\&HT) techniques hold great potential for making materials research more productive, more thorough, and less wasteful. However, significant barriers prevent the widespread adoption of these revolutionary methods. Through creative, cost-effective measurement solutions, and with an eye towards fruitful collaboration, the NIST Combinatorial Methods Center (NCMC) strives to ease the acquisition of $C \& H T$ techniques by the materials research community.
\end{abstract}

\section{Cher H. Davis and Michael J. Fasolka}

$\mathrm{N}$ ow in its third year, the NIST Combinatorial Methods Center continues to be a sought after partner among industry, government laboratories, and academics interested in acquiring C\&HT research capabilities for materials research. Indeed, the NCMC consortium currently includes 22 member institutions (see table) representing a broad cross-section of the materials research sector.

The NCMC is successful because it serves the combinatorial materials research community on several levels. Our foundation is the extensive suite of NCMC technologies, which provide measurement solutions to parties interested in acquiring C\&HT capabilities. Many of these methods are described elsewhere in this report, as identified by the NCMC symbol (see top right corner).

Technology transfer efforts complement our methods foundation, as evidenced by the many institutions that have adopted NCMC techniques. For example, Air Products, Rhodia, National Starch (ICI), Dow, Procter and Gamble, and Eastman, plus many universities, have replicated NCMC devices in their laboratories. In this respect, NCMC Focused Projects are a new paradigm for NIST/industry partnership, which are extremely effective for both methods development and technology transfer.

\begin{abstract}
"The focused project approach is particularly effective since it allows active collaboration with industry, allowing real needs to be addressed, and resulting technology transferred. The [NCMC] is at the leading edge of combi-based material science discovery, and this reflects both the quality of the research program and of the people."

— Dr. J. Carroll, ICI/National Starch, Strategic Technology Group
\end{abstract}

Under a Focused Project, NIST scientists and 2-3 member companies collaborate to develop a particular C\&HT measurement solution. While the research is co-funded by industry members, we avoid the study of proprietary materials and all results are published; this allows the research to have the broadest impact. Currently, there are two Focused Projects. In the first, NIST is working with Procter and Gamble and National Starch (ICI) to produce HT microfluidic measurements of interfacial tension. The second Focused Project, sponsored by Intel and National Starch (ICI), will produce C\&HT methods that gauge the performance of epoxy adhesives for flip chip electronics applications. Several other Focused Projects are in development.

New scientific endeavors (such as C\&HT materials research) gain momentum when stake-holding parties are brought together. Accordingly, to advance the field, the NCMC invests itself in community forming activities aimed at information transfer and goal consolidation among institutions interested in C\&HT research. Industry workshops play a major role in this endeavor, and in FY04 we hosted the 4th and 5th additions to the NCMC workshop series, focused on Polymer Formulations and Processing and Characterization, respectively.

"The NCMC provides a unique platform to exchange information and ideas with other member companies who form a part of this consortium."

D. Bhattacharya, Global Coatings Application Development, Eastman Chemical Co.

Moreover, we have been very active in organizing symposia at national conferences. In FY04 alone, NCMC staff developed and organized multi-day symposia on C\&HT methods for the Materials Research Society, the American Chemical Society, the Adhesion Society, and the Knowledge Foundation.

"I have heard nothing but praise from all who attended the recent NCMC conference. . .NIST did a superb job organizing the symposia, selecting thought-provoking topics, and leading good general discussions."

- Personal observation of J. Dias, ExxonMobil; Chair ACS PMSE

For more information on the NCMC, C\&HT technologies, or post-doctoral research opportunities, see our website at http://www.nist.gov/combi.

\begin{tabular}{|c|c|}
\hline \multicolumn{2}{|c|}{ NCMC Members (*New in FY2004): } \\
\hline $3 M$ & Honeywell International \\
\hline Accelrys & Hysitron International* \\
\hline ce Res & Intel* \\
\hline $\begin{array}{l}\text { Air Products \& Chemicals } \\
\text { Akzo Nobel }\end{array}$ & $\begin{array}{c}\text { ICI/National Starch \& Chemicals } \\
\text { Michelin }\end{array}$ \\
\hline Atofina Chemicals* & $P P G$ Industries \\
\hline$B P^{*}$ & Procter \& Gamble \\
\hline & Rhodia \\
\hline$r$ Polyme & Sealed Air Corp* \\
\hline Dow Chemical Company & Symyx* \\
\hline Eastman Chemical* & Mississippi \\
\hline ExxonMobil Research & Veeco/Digital Instruments* \\
\hline
\end{tabular}

\section{Contributors and Collaborators}

K.L. Beers, C.M. Stafford, A. Karim, E.J. Amis, The Multivariant Measurement Methods Group (Polymers Division, NIST) 


\section{Organizational Charts}

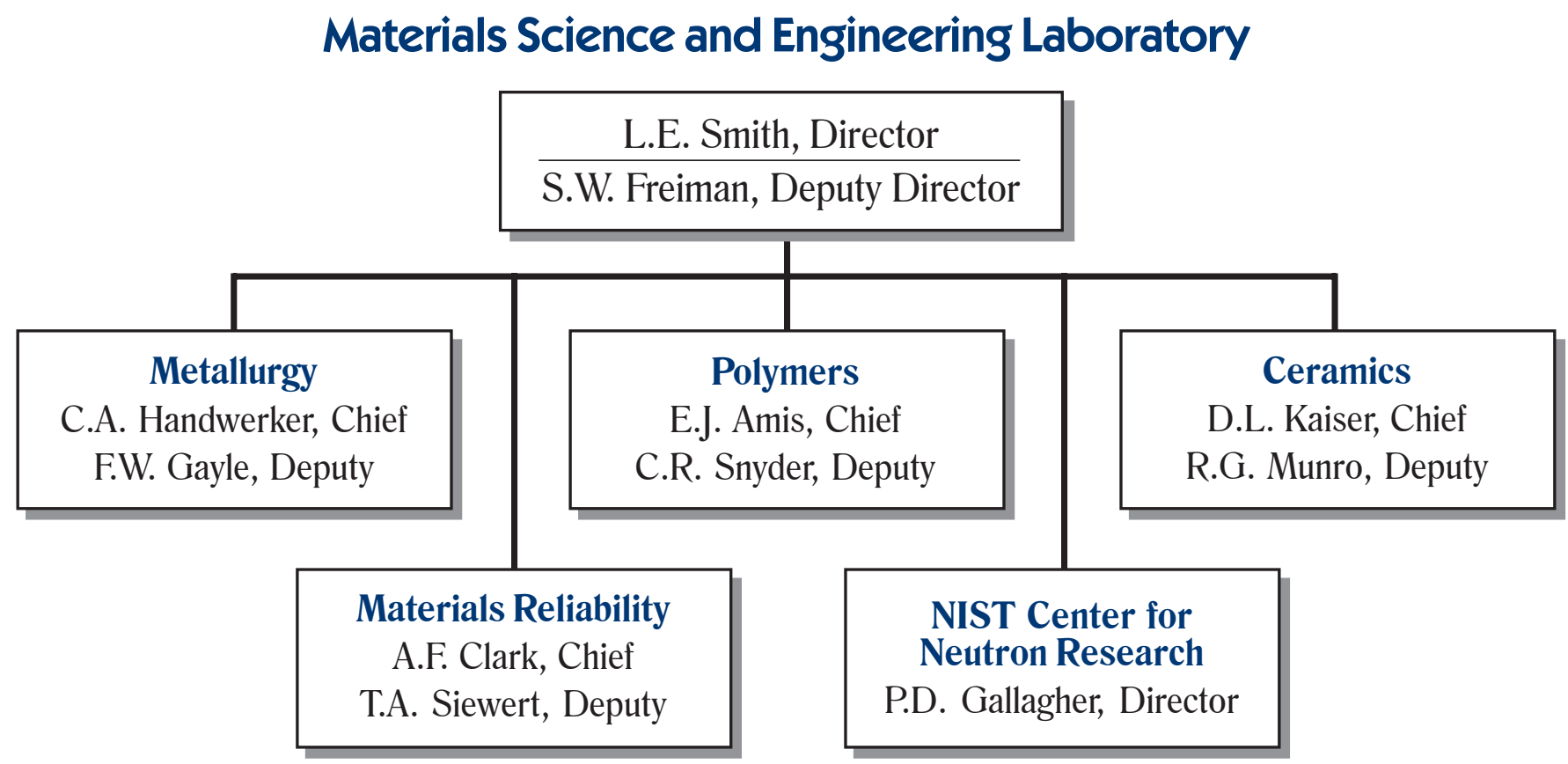

\section{National Institute of Standards and Technology}

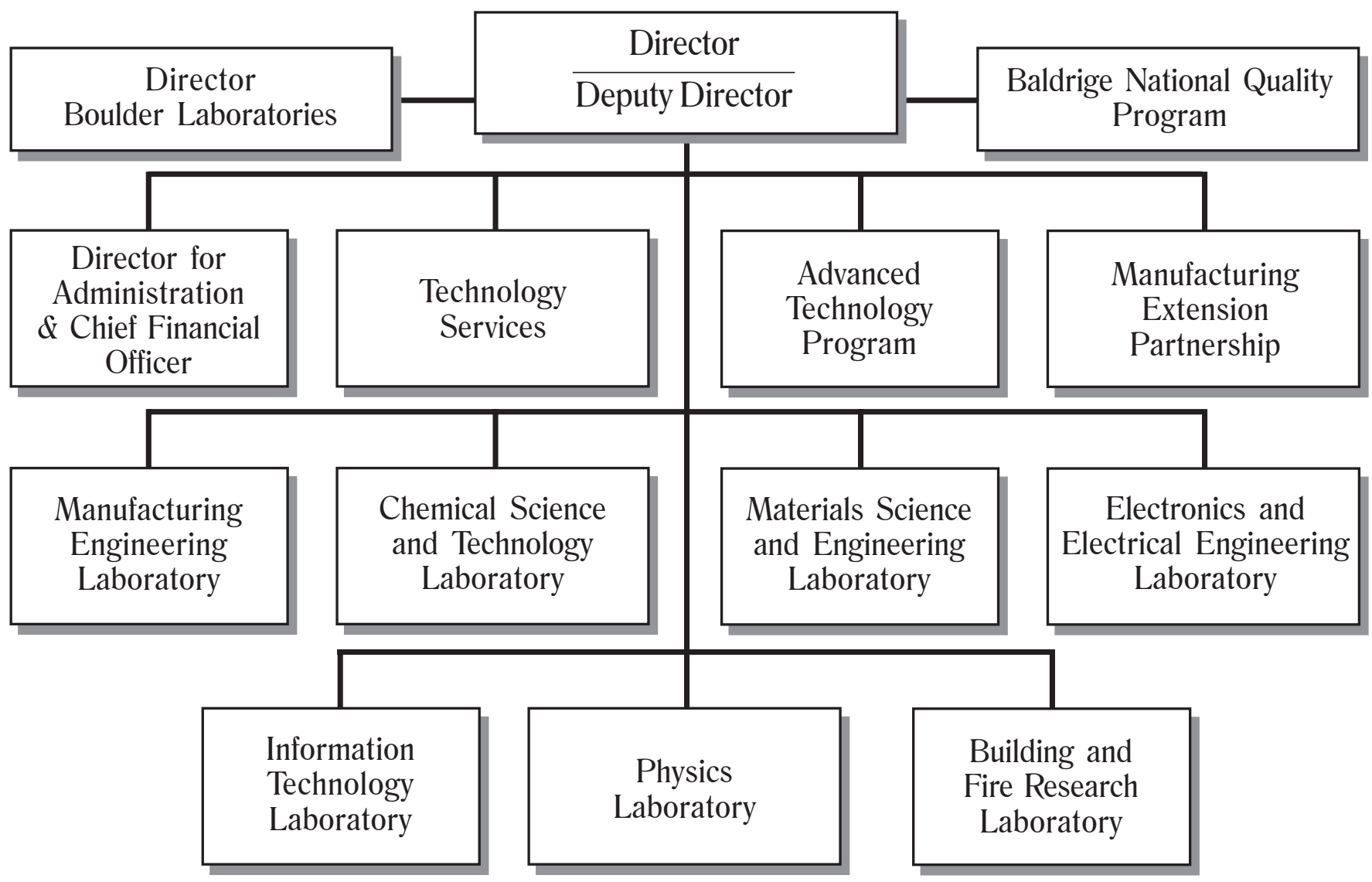



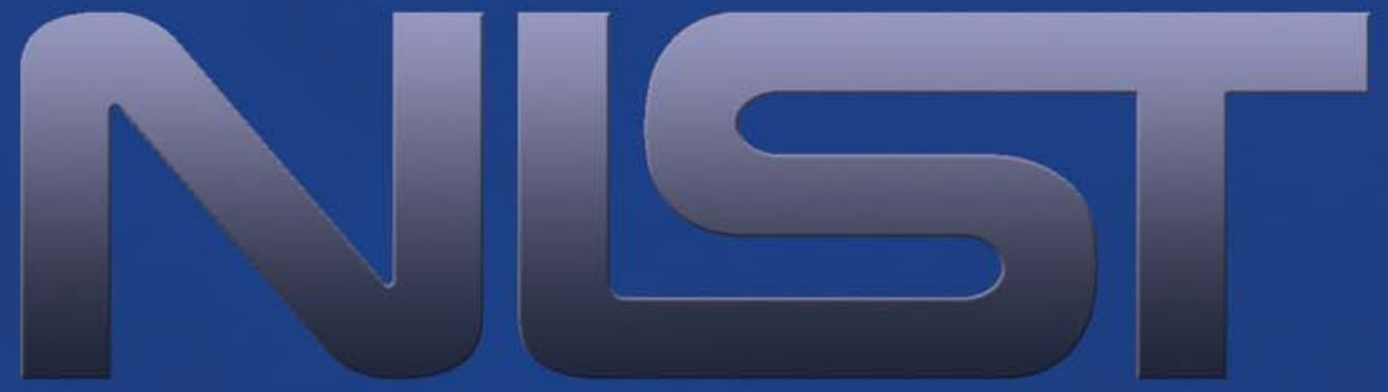

(1)

Ifllinisii

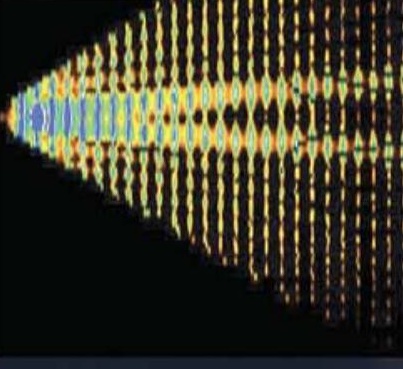

